\title{
Advancements in Monte Carlo dose calculations for prostate and breast permanent implant brachytherapy
}

by

Nelson Miksys

A thesis submitted to the Faculty of Graduate and Postdoctoral Affairs in partial fulfillment of the requirements for the degree of

Doctor of Philosophy

in

Physics (Medical Physics)

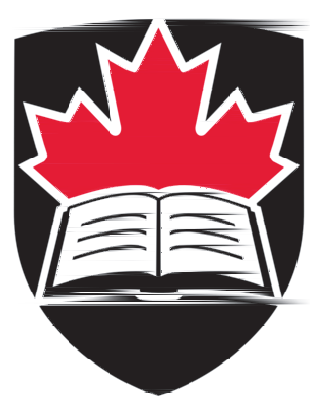

Ottawa-Carleton Institute for Physics

Department of Physics

Carleton University

Ottawa, Ontario, Canada

2016

(C)2016 Nelson Miksys 


\section{Abstract}

Monte Carlo (MC) simulations of radiation transport may provide more accurate estimates of dose delivered to permanent implant brachytherapy patients compared to the current clinical AAPM TG-43 dose calculation paradigm. However, MC dose calculations are burdened by considerable sensitivity to several required modelling assumptions, especially with the low-energy photon sources typical of permanent implant brachytherapy (20-30 keV).

MC simulations require a detailed virtual model of the patient, often derived from post-treatment CT images which contain imaging artifacts due to the presence of the brachytherapy sources during image acquisition. For the first time, several metallic artifact reduction algorithms, of varied approach, are explored in phantom and clinical prostate brachytherapy CT images to determine their ability to mitigate artifacts and to quantify the sensitivity on the resulting dose calculations.

Permanent implant breast brachytherapy presents a particular challenge to model due to the radiologically different adipose and fibrogland soft tissues in and near the treatment volume. Further, the geometry of a breast treatment is especially non-water like which suggests the clinical TG-43 dose calculation paradigm may yield significantly inaccurate results. The dose calculation sensitivity to metallic artifact reduction, tissue differentiation approach and simulated tissue composition are ex-

plored in permanent implant breast brachytherapy clinical patient data, in addition 
to presenting differences between realistic and TG-43 conditions for various target and organ-at-risk dosimetric endpoints.

This work presents the largest cohort of permanent implant prostate brachytherapy MC dose calculations published to-date, providing quantitative differences between realistic and TG-43 conditions and a detailed sensitivity analysis of organ-atrisk simulated tissue composition, calcification modelling approach and calcification tissue composition.

Clinical radiotherapy is increasingly considering radiobiological endpoints as a metric of treatment quality. Various radiobiological indices are evaluated using a large cohort of prostate brachytherapy dose calculations to explore the differences between these models and differences between applying TG-43 or realistic tissue MC dose calculations. This work identifies challenges related to applying dose calculations in heterogeneous tissue models to existing radiobiological models.

The contributions of this thesis improve the understanding of realistic brachytherapy dose calculations, pave the way for the clinical adoption of Monte Carlo dose calculations and challenge and build upon the current frontier of radiobiological modelling for permanent implant brachytherapy. 


\section{Acknowledgements}

I would like to express my deepest gratitude to my partner Jenna Glover for her continued support, patience and love during my time at Carleton University.

My supervisor Dr. Rowan Thomson has been an incredible inspiration during my studies, she has demonstrated a stellar work ethic, a genuine passion for scientific research and a unwavering commitment to developing her students into the best possible graduates they can be. Agreeing to have Rowan as a PhD supervisor was the best professional decision I have made so far.

I thank my scientific collaborators for their efforts to review and provide insights to strengthen manuscripts; Dr Luc Beaulieu, Dr Joanna Cygler, Dr Justin Sutherland, Mehan Haidari, Dr Jean-Michel Caudrelier, Dr Eric Vigneault and Dr Andre-Guy Martin.

I sincerely appreciate the discussions and technical assistance from my peers and colleagues during my time at Carleton; Dr Justin Sutherland, Marielle Lesperance, Dr Dal Granville, Martin Martinov, Patty Oliver, Mehan Haidari and Stephen Deering

I would like to thank my PhD committee for their advice and efforts: Dr Rowan Thomson, Dr David Rogers and Dr Andre Longtin. 
Finally, I am very grateful for the various sources of financial support which were awarded to me during my PhD studies: the Carleton University Research Office and Department of Physics, the Ontario Graduate Scholarship and the KiwanisMarwah Graduate Scholarship. In addition, this research was supported by the Canadian Cancer Society Research Institute, Natural Sciences and Engineering Research Council of Canada, Canada Research Chair, and Ministry of Research and Innovation of Ontario Early Researcher Award. 


\section{Statement of originality}

This thesis is a summary of the most significant contributions of the author's research while enrolled in the Ph.D. program at Carleton University. Many of the results have been published as manuscripts in peer reviewed journals and presented at national and international conferences.

Dr Rowan Thomson supervised the author and contributed to all aspects of each study, through guidance and manuscript revision. Dr Luc Beaulieu and Dr Joanna Cygler contributed to study design and manuscript revision for select publications identified below. Additional scientific collaborators, identified as authors below, contributed to manuscript revision prior to journal submission. Otherwise, the author of this thesis is responsible for all study design, development of methodology, computational work, data analysis, publication writing and revisions.

\section{Published manuscripts}

1. Miksys, N., Xu, C., Beaulieu, L., Thomson, R. M. Development of virtual patient models for permanent implant brachytherapy Monte Carlo dose calculations: interdependence of $\mathrm{CT}$ image artifact mitigation and tissue assignment. Phys. Med. Biol., 60:6039-6062, 2015

- The results of this manuscript are described in Chapter 3 
- Select results of this manuscript, and similar works, were presented at AAPM annual conference (Indianapolis 2013) and COMP annual conference (Montreal 2013)

2. Miksys, N., Cygler, J. E., Caudrelier, J. M., Thomson, R. M. Patient-specific Monte Carlo dose calculations for Pd-103 breast brachytherapy. Phys. Med. Biol., 61(7):2705, 2016

- The results of this manuscript are described in Chapter 4

- Select results of this manuscript were presented at COMP annual conference (Montreal 2013)

\section{Manuscripts under review and in preparation}

1. Miksys, N. Vigneault, E. Martin, A. G., Beaulieu, L., Thomson, R. M., Large scale retrospective Monte Carlo dosimetric study for permanent implant prostate brachytherapy. Under review with International Journal of Radiation Oncology, Biology, Physics.: submitted July 2016

- The results of this manuscript are described in Chapter 5

- Select results of this manuscript were presented at International Workshop on Monte Carlo Techniques in Medical Physics (Quebec City, 2014), IUPESM World Congress (Toronto 2015) and ABS World Congress (San Francisco 2016)

2. Miksys, N., Beaulieu, L., Thomson, R. M., Radiobiological modelling for I-125 permanent implant prostate brachytherapy Monte Carlo dose calculations. To be submitted to Medical Physics in September 2016

- The results of this manuscript are described in Chapter 6 


\section{List of Abbreviations}

CPE ........ Charged Particle Equilibrium

CT $\ldots \ldots \ldots$..... Computed Tomography

CTV ......... Clinical Target Volume

DHI $\ldots . . . \ldots$ Dose Homogeneity Index

HU ......... Hounsfield Unit

KERMA …. Kinetic Energy Released per unit Mass

MAR ........ Metallic Artifact Reduction

MBDCA …. Model Based Dose Calculation Algorithm

MC .......... Monte Carlo

PIPB ....... Permanent Implant Prostate Brachytherapy

PTV ......... Planning Target Volume

TAS ........ Tissue Assignment Scheme

TG-43 ....... AAPM Task Group 43

TG-186 ...... AAPM Task Group 186

STR ........ Simple Threshold Replacement 


\section{Contents}

1 Introduction 1

1.1 Brachytherapy ....................... . . . 2

1.1.1 Prostate Cancer . . . . . . . . . . . . . . . . 3

1.1.2 Breast Cancer . . . . . . . . . . . . . . . 5

1.2 AAPM TG-43 Dose Calculations . . . . . . . . . . . . . 6

1.3 Model Based Dose Calculations . . . . . . . . . . . . . . 8

1.4 Monte Carlo Dose Calculations . . . . . . . . . . . . . . . . . . . . . 10

1.5 Radiobiological Modelling . . . . . . . . . . . . . . . 11

1.6 Thesis Purpose . . . . . . . . . . . . . . . . . 12

1.7 Thesis Outline. . . . . . . . . . . . . . . . . 12

2 Monte Carlo Dose Calculation Methodology 15

2.1 BrachyDose Simulations . . . . . . . . . . . . . . 16

2.2 Development of Virtual Patient Models . . . . . . . . . . . . . . . 17

2.3 Dose and Statistical Uncertainty Analysis . . . . . . . . . . . . 20

3 Metallic Artifact Reduction in CT Images 22

3.1 Introduction . . . . . . . . . . . . . . . . 23

3.2 Methods ........................... 24 
3.2.1 Metallic Artifact Reduction . . . . . . . . . . . . . . . 24

3.2 .2 Tissue Assignment . . . . . . . . . . . . . . . . . . . . 28

3.2 .3 Dose Calculations . . . . . . . . . . . . . . . . . . . . . 32

3.3 Results . . . . . . . . . . . . . . . . . . . . . . . . . . 32

3.3.1 Metallic Artifact Reduction . . . . . . . . . . . . . . . 32

3.3 .2 Tissue Assignment . . . . . . . . . . . . . . . . . . 37

3.3.3 Dose Distributions . . . . . . . . . . . . . . . . . . . . . 40

3.4 Discussion . . . . . . . . . . . . . . . . . . . 46

3.5 Conclusions . . . . . . . . . . . . . . . . . . . . . 51

4 Monte Carlo Dose Calculations for Breast Brachytherapy 53

4.1 Introduction . . . . . . . . . . . . . . . . 54

4.2 Methods . . . . . . . . . . . . . . . . . 57

$4.2 .1 \quad$ Patient Data . . . . . . . . . . . . . . . . 57

4.2 .2 Metallic artifact reduction . . . . . . . . . . . . . 57

4.2 .3 Tissue assignment . . . . . . . . . . . . . . . . . . . . 59

4.2 .4 Dose calculations . . . . . . . . . . . . . . . . . . 64

4.3 Results . . . . . . . . . . . . . . . . . . . . . . 64

4.3.1 Metallic artifact reduction . . . . . . . . . . . . . . . 64

4.3.2 Virtual patient model tissue assignment . . . . . . . . . 67

4.3 .3 Dose calculations . . . . . . . . . . . . . . . . . . 69

4.4 Discussion . . . . . . . . . . . . . . . . . . . . . . 80

4.5 Conclusions . . . . . . . . . . . . . . . . . . . 87

5 Monte Carlo Dose Calculations for Prostate Brachytherapy $\quad 89$

5.1 Introduction . . . . . . . . . . . . . . . 90 
5.2 Methods and Materials . . . . . . . . . . . . . . . . . . . . 90

5.2.1 Patient Data . . . . . . . . . . . . . . . . . 90

5.2.2 Metallic Artifact Reduction . . . . . . . . . . . . . . . . 91

5.2.3 Virtual Patient Model . . . . . . . . . . . . . . . . . . . 91

5.2.4 Sensitivity Analysis . . . . . . . . . . . . . . . . . . . 92

5.2.5 Monte Carlo Dose Calculations . . . . . . . . . . . . . . . 95

5.3 Results . . . . . . . . . . . . . . . . . . 96

5.3.1 Large Patient Cohort . . . . . . . . . . . . . . . . . . . 96

5.3.2 Sensitivity Analysis . . . . . . . . . . . . . . . . . 99

5.4 Discussion . . . . . . . . . . . . . . . . . . . 103

5.5 Conclusions .......................... 105

6 Radiobiological Modelling for Prostate Brachytherapy 106

6.1 Introduction . . . . . . . . . . . . . . . . 107

6.2 Methods ......................... 107

6.2.1 Radiobiological models . . . . . . . . . . . . . . . 107

6.2.2 Coupling MBDCAs with Radiobiological Models . . . . . . . . 112

6.3 Results . . . . . . . . . . . . . . . . . . . . . . 115

6.3.1 Example patients with calcifications . . . . . . . . . 115

6.3.2 Large Patient Cohort . . . . . . . . . . . . . . . . 116

6.4 Discussion . . . . . . . . . . . . . . . . . . . 123

6.4.1 Model Based Dose Calculations compared with TG-43 . . . . 123

6.4.2 Choice of radiobiological model . . . . . . . . . . . . . 124

6.4.3 Sensitivity to assumed biological parameters . . . . . . . . 126

6.4.4 Radiobiological modelling advancements . . . . . . . . . . . . 128 
6.5 Conclusions . . . . . . . . . . . . . . . . . . . . . . 130

7 Summary and Outlook 132

7.1 Scientific Challenges Addressed in this Thesis . . . . . . . . . . . . 133

7.2 Summary of Chapter Conclusions . . . . . . . . . . . . . . . . . 134

7.2.1 Chapter 3 - Metallic Artifact Reduction . . . . . . . . . . . . 134

7.2.2 Chapter 4 - Breast Dose Calculations . . . . . . . . . . . . . 135

7.2.3 Chapter 5 - Prostate Dose Calculations . . . . . . . . . . . 136

7.2.4 Chapter 6 - Prostate Radiobiological Modelling . . . . . . . . 137

7.2.5 Outlook and Future Research Possibilities . . . . . . . . . . . 138

$\begin{array}{ll}\text { References } & 140\end{array}$ 


\section{List of Tables}

1.1 Brachytherapy Radioisotopes ................ 3

2.1 Tissue Compositions Part $1 \ldots \ldots$. . . . . . . . . . 18

2.2 Tissue Compositions Part 2 . . . . . . . . . . . . . . . . . . 19

3.1 Tissue Assignment Schemes . . . . . . . . . . . . . . . . . 30

3.2 CT Number to Mass Density Calibration . . . . . . . . . . . . . 31

3.3 CT Numbers in ROIs . . . . . . . . . . . . . . . . . . 37

3.4 Tissue Assignment Distributions . . . . . . . . . . . . . . . . . 38

3.5 Phantom Dose Metrics . . . . . . . . . . . . . . . . . . . . 42

3.6 TG-186 Reference Model Dose Metrics . . . . . . . . . . . . . . . . . 42

3.7 Prostate Patient Dose Metrics . . . . . . . . . . . . . . . 43

4.1 Tissue Assignment Schemes . . . . . . . . . . . . . . . . . 60

4.2 CT Number to Mass Density Calibration . . . . . . . . . . . . . . 61

4.3 Tissue Assignment Distributions . . . . . . . . . . . . . . . . . . 68

4.4 Breast Patient PTV Dose Metrics . . . . . . . . . . . . . . . . 72

4.5 Breast Patient Skin Dose Metrics . . . . . . . . . . . . . . 73

5.1 Patient Cohort Characteristics . . . . . . . . . . . . . . . . 91

5.2 Tissue Assignment Schemes . . . . . . . . . . . . . . . . 94 
5.3 Dose Metrics . . . . . . . . . . . . . . . . . . . . . . . . . . . . . . . 98

6.1 Radiobiological Parameters . . . . . . . . . . . . . . . . . . 108

6.2 Summary of Radiobiological Models . . . . . . . . . . . . . . . . . . . 113

6.3 Example Patient EUBEDs and TCPs . . . . . . . . . . . . . 118

6.4 Large Cohort Biological Doses and TCPs . . . . . . . . . . . . . . . . 119 


\section{List of Figures}

1.1 Brachytherapy Source (Seed) Schematics . . . . . . . . . . . . . 4

1.2 Example Workflow . . . . . . . . . . . . . . . . . . . . . . 14

2.1 DVH Dose Metric Schematic . . . . . . . . . . . . . . . 21

3.1 CTV CT Number Histogram . . . . . . . . . . . . . . . . 25

3.2 Phantom and Prostate MAR images . . . . . . . . . . . . . 33

3.3 Phantom CT Line Profile . . . . . . . . . . . . . . . . . . . 34

3.4 Prostate CT Line Profile . . . . . . . . . . . . . . . . . . . . 35

3.5 Dose Line Profile . . . . . . . . . . . . . . . . . . . . . . 44

3.6 Dose Ratios . . . . . . . . . . . . . . . . . . . . . . 46

4.1 Breast Tissue Mass Energy Absorption Coefficients . . . . . . . . . . 56

4.2 MAR Applied to Breast CT Images . . . . . . . . . . . . . 63

4.3 Breast CT Line Profile . . . . . . . . . . . . . . . 66

4.4 CT Numbers in ROIs . . . . . . . . . . . . . . . . . . . . . 67

4.5 Dose Sensitivity - MAR . . . . . . . . . . . . . . 71

4.6 Dose Sensitivity - Tissue Assignment . . . . . . . . . . . . . 75

4.7 Dose Sensitivity - Tissue Composition . . . . . . . . . . . . . . . 77

4.8 MC vs TG43 Doses . . . . . . . . . . . . . . . . . . . . 79

4.9 Dose Sensitivity Summary . . . . . . . . . . . . . . . 80 
5.1 Prostate Mass Energy Absorption Coefficients . . . . . . . . . . . . 93

5.2 Average Target and OAR DVHs . . . . . . . . . . . . . . 96

5.3 Example Prostate Patients . . . . . . . . . . . . . . . . . . . 100

5.4 Dose Metric Trends . . . . . . . . . . . . . . . . . . . . . . . . . . . 101

5.5 Dose Calculation Sensitivity Summary . . . . . . . . . . . . . . 102

6.1 Example Patient Doses . . . . . . . . . . . . . . . . . . . 114

6.2 Low Doses Identified by EUBED . . . . . . . . . . . . . . . . 117

6.3 Large Cohort Biological Doses and TCPs . . . . . . . . . . . . . . 120

6.4 Relationship Between Biological Dose Models . . . . . . . . . . . . . 122

6.5 EUBED and TCP Sensitivity to Dose Cut-Off . . . . . . . . . . 125

6.6 EUBED and TCP Sensitivity to Parameters . . . . . . . . . . . . 127

6.7 EUBED and TCP Sensitivity to Number of Cells . . . . . . . . . 128 


\section{Chapter 1}

\section{Introduction}

This Chapter introduces the scientific challenges related to permanent implant brachytherapy Monte Carlo dose calculations which are the focus of this thesis. This includes several issues related to developing accurate virtual patient models which are needed for these dose calculations. This work explores these state of the art dose calculations in clinical treatment data for a better understanding of delivered radiation dose distributions and addresses new issues related to incorporating these dose calculations into existing models of biological effect. 


\subsection{Brachytherapy}

Cancer is the leading cause of mortality in Canada, responsible for about a third of all deaths. ${ }^{1}$ Treatment approaches vary with cancer type and stage of disease progression, but generally include one or more of the following: active monitoring, surgical resection of the tumour, radiation therapy or chemotherapy.

Radiation therapy aims to kill cancer cells by delivering radiation dose (energy imparted to a medium per unit mass, unit: $\mathrm{J} / \mathrm{kg}=\mathrm{Gy}$ ) which, if enough dose is delivered, can cause cell death. Since the radiation will also damage healthy tissues, a critical aspect of radiation therapy is a determination of the spatial distribution of radiation dose to assist in maximizing the therapeutic ratio for treatment planning and verify the quality of treatment after delivery.

The most common type of radiation therapy for cancer treatment uses an external radiation source to treat diseased tissue, but often additionally irradiates a large volume of healthy tissue. Brachytherapy is an alternate form of radiation therapy which uses one or more radioactive sources positioned near or inside the body and offers conformal highly heterogeneous dose distributions which may better spare dose to proximal organs-at-risk. Although different approaches to brachytherapy exist, most are interstitial procedures which involve surgically positioning radioactive sources in soft tissues. Brachytherapy treatments can be stratified by the rate at which they deliver radiation dose to the patient; with high dose rate treatments delivering above $12 \mathrm{~Gy} / \mathrm{hr},{ }^{2}$ and low dose rate permanent implants having initial dose rates of 0.3 to $2 \mathrm{~Gy} / \mathrm{hr}^{2}{ }^{2}$ Unlike external treatments which typically deliver radiation generated with a linear accelerator, brachytherapy sources mostly rely on cyclotron or nuclear reactor generated radioisotopes. A summary of some typical low dose rate brachytherapy radioisotopes, and the characteristics of their emitted photon 
radiation, is described in Table 1.1 .

Table 1.1: Summary of common brachytherapy radioisotopes. ${ }^{3}$

\begin{tabular}{ccc}
\hline \hline Isotope & $\begin{array}{c}\text { Mean Photon } \\
\text { Energy }[\mathrm{MeV}]\end{array}$ & Half Life \\
\hline${ }^{103} \mathrm{Pd}$ & 0.021 & 17 days \\
${ }^{125} \mathrm{I}$ & 0.028 & 59.4 days \\
${ }^{192} \mathrm{Ir}$ & 0.38 & 73.8 days \\
${ }^{198} \mathrm{Au}$ & 0.41 & 2.7 days \\
${ }^{137} \mathrm{Cs}$ & 0.66 & 30 years \\
${ }^{60} \mathrm{Co}$ & 1.25 & 5.26 years \\
\hline \hline
\end{tabular}

Permanent implant brachytherapy utilizes commercially developed sources, often called seeds, which are generally a single radioactive isotope enclosed in an inert casing. These seeds vary in design across manufacturers, but are generally cylindrical, a few millimetres in length and less than a millimetre in diameter. For low energy radioisotopes such as ${ }^{125} \mathrm{I}$ and ${ }^{103} \mathrm{Pd}$, significant attenuation occurs in the seed casing, leading to a model-specific photon energy spectrum escaping the source. For this reason, accurate dose calculations rely on the development of accurate source models (Figure 1.1).

\subsubsection{Prostate Cancer}

Males have a 45\% chance to develop cancer in their lifetime. In 2015, 100500 new cancers were diagnosed in Canadian men, and prostate cancer was the most frequent at $24 \%$ of cases. The Canadian Cancer Society estimates that $13 \%$ of men will develop, and $4 \%$ will die from prostate cancer. ${ }^{1}$

Treating early stage prostate cancer with brachytherapy is well established and began in Canada in the 1990s. ${ }^{5}$ As of 2012, over 13000 permanent implant 
Amersham OncoSeed 6711 (I-125)

AAPM TG-43 Dose Rate Constant $=0.965 \mathrm{cGy} \mathrm{hr}^{-1} \mathrm{U}^{-1}$

Source in Chapter 3 \& Chapter 5

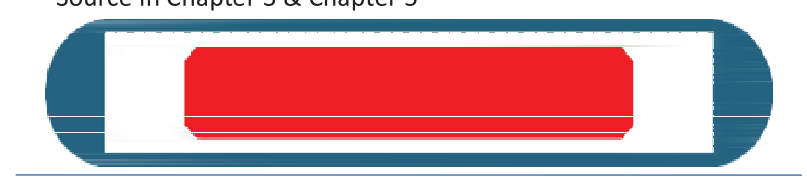

Theragenics TheraSeed 200 (Pd-103)

Dose Rate Constant $=0.686 \mathrm{cGy} \mathrm{hr}^{-1} \mathrm{U}^{-1}$

Source in Chapter 4

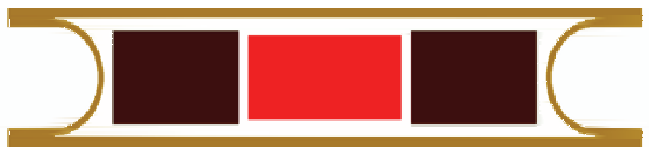

DRAXIMAGE BrachySeed LS-1 (I-125)

AAPM TG-43 Dose Rate Constant $=0.972 \mathrm{cGy} \mathrm{hr}^{-1} \mathrm{U}^{-1}$

Source in Chapter 5

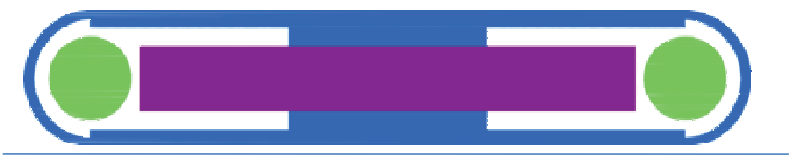

Nucletron SelectSeed(I-125)

AAPM TG-43 Dose Rate Constant $=0.987 \mathrm{cGy} \mathrm{hr}^{-1} \mathrm{U}^{-1}$

Source in Chapter 5

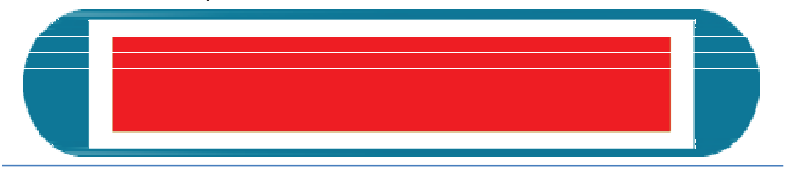

Mills Bio Pharm ProstaSeed 125SL (I-125)

AAPM TG-43 Dose Rate Constant $=0.953 \mathrm{cGy} \mathrm{hr} \mathrm{r}^{-1} \mathrm{U}^{-1}$

Source in Chapter 5

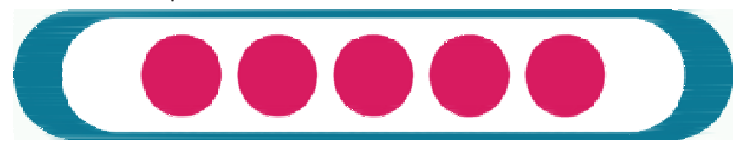

Figure 1.1: ${ }^{125} \mathrm{I}$ and ${ }^{103} \mathrm{Pd}$ permanent implant brachytherapy sources used in this thesis. Further details on the virtual seed model dimensions and materials depicted in the schematics can be obtained from The CLRP TG-43 Parameter Database for Brachytherapy. ${ }^{4}$

prostate brachytherapy treatments have been performed in Canada. ${ }^{5}$ Low and intermediate risk prostate cancers are generally delivered mono-therapy permanent implant brachytherapy which shows 5-10 year biochemical free survival rates as low as $85 \%(61 \%)$ and as high as $98 \%$ (98\%) for low (intermediate) risk groups. ${ }^{6-10}$

Permanent implant prostate brachytherapy treatments are outpatient pro- 
cedures which are about an hour in length and require general or local anesthesia. With the patient in lithotomy position, seeds are implanted transperineally through template constrained needles under ultra sound guidance using a transrectal ultrasound probe. Some centres use a catheter to help differentiate the urethra and prostate. More comprehensive descriptions of the Canadian permanent implant prostate brachytherapy procedures are available. ${ }^{5}$ Postoperative CT images are acquired to determine the implanted seed positions as they will differ from the planned positions. Using these post-implant CT images, follow up radiation dose calculations are performed to help determine treatment quality.

\subsubsection{Breast Cancer}

Females have a 42\% chance to develop cancer in their lifetime. In 2015, 96400 new cancers were diagnosed in Canadian women, and breast cancer was the most frequent at $26 \%$ of cases. In the 1990s Canadian breast cancer diagnoses increased as mammography screening programs were introduced, and declined in the 2000 s as hormone replacement therapy was largely discontinued in post-menopausal women. As of 2010, the Canadian Cancer Society estimates that $11 \%$ of women will develop, and $3 \%$ will die from breast cancer. ${ }^{1}$

Typically, treatments for early stage breast cancer will include breast-conserving surgery and adjuvant external beam radiotherapy to treat the whole breast. This treatment approach requires frequent visits to the clinic, occasionally resulting in patients opting for a mastectomy for convenience, ${ }^{11}$ and whole breast irradiation can lead to severe skin reactions. ${ }^{12}$ Treating breast cancer with brachytherapy is a rel-

atively new procedure, with the first treatments in Canada in 2004. ${ }^{13}$ Similar to the traditional treatment approach, permanent breast seed implants (PBSI) are an 
adjuvant radiotherapy after local resection of the tumour, but differ by delivering radiation dose locally around the surgical bed where most reoccurant disease occurs. PBSI is advantageous as it requires only a single treatment and lowers the risk of skin effects. ${ }^{14}$ Preliminary outcomes suggest that PBSI can offer a 5-year disease free rate of $96 \%$ which is comparable to traditional whole breast irradiation. ${ }^{15}$

\subsection{AAPM TG-43 Dose Calculations}

A critical step to all radiation therapy, including brachytherapy, is a determination of the radiation dose distribution delivered to the patient. Dose calculations are needed for treatment planning and for post-treatment analysis. To illustrate the importance of post-implant dosimetry, studies of permanent implant prostate brachytherapy have identified dosimetric descriptors such as the minimum dose delivered to $90 \%$ of the

volume $\left(\mathrm{D}_{90}\right)$, or the percentage of the volume which is delivered at least the prescription dose $\left(\mathrm{V}_{100}\right)$, to be strong predictors of recurrence-free local disease control. ${ }^{16-18}$

In brachytherapy, the current clinical dose calculation approach is based on an analytic formalism defined by AAPM Task Group Report $43,{ }^{19}$ and its updates. ${ }^{20,21}$ The TG-43 formalism calculates the dose distribution around single sources in homogeneous water with sufficient surrounding scatter volume. Then, by using the known spatial distribution of sources obtained from post-implant imaging, the total patient dose is calculated by summing the dose contributions from each source. The TG-43 dose calculations are advantageous as they are simple to implement, reproducible and require minimal computational power. Several commercial TG-43 based treatment planning and dose calculation software packages are routinely used in Canadian clinics, for example, Theratronics THERAPLAN and Nucletron SPOT-PRO. ${ }^{22}$ 
The TG-43 dose calculations have an uncertainty of several percent. ${ }^{20}$ While capable of describing the cylindrically symmetric dose distribution around a source, post-implant imaging is typically unable to determine seed orientation, often resulting in the implementation of hybrid or isotropic source approximations. TG-43 suggests that isotropic approximations generally result in single-source dose errors of less than $2 \%$ beyond $1 \mathrm{~cm}$ from the source, but errors as large as $74 \%$ can exist close to the source. However, when considering the effects from multiple sources on overall patient doses from permanent implant prostate brachytherapy, seed orientation has negligible effect on overall target volume doses..$^{23,24}$

TG-43 relies on defining a dose-rate constant for each low energy source which acts as a scaling factor in the dose calculation. Practically, these consensus dose-rate constants are defined as an average of experimental and computational published values. Experimental values, often based on TLD measurements, suffer from positioning, calibration and energy correction uncertainties, which TG-43 estimates may amount to an overall uncertainty of up to $9 \%$. On the other hand, computational values suffer from uncertainties in assumed cross section, source geometry and radioisotope decay spectra, which TG-43 estimates may amount to an overall uncertainty of up to $5 \%$. Overall, TG-43 estimates the uncertainty on dose calculations to be $6-7 \%$ and acknowledges the possibility of further uncertainty due to irregularities in the manufacturing of seed casings.

Beyond the uncertainties in defining the TG- 43 data, these dose calculations are fundamentally inaccurate because of three key assumptions; the patient is made of homogeneous water, the radiation from one source does not scatter or attenuate in the other sources, and there is substantial scattering volume around each source. ${ }^{25}$ Thus, there is a need to develop, and integrate into clinics, more accurate dose calculations for brachytherapy. 


\subsection{Model Based Dose Calculations}

There is a movement towards the clinical adoption of more advanced model based dose calculation algorithms (MBDCAs) $)^{25-27}$ for brachytherapy, which are able to calculate dose in non-water mediums such as soft tissues, bone, lung or calcifications, and in inorganic materials such as gynecological applicators or prosthetic implants. However, these MBDCAs fundamentally rely on the knowledge of cross sections and material compositions as well as accurate modelling of radiation physics, source geometries, source positions and patient geometries.

The accurate development of virtual patient models is an outstanding challenge for permanent implant brachytherapy MBDCAs, but basic modelling guidelines are provided in TG-186. ${ }^{26}$ Practically, patient models can be derived from post-implant CT images which yield a voxelized geometry sharing the same grid as the CT image. Post-implant CT images contain bright spots around sources and streaking artifacts due to the presence of the metallic brachytherapy seeds during the CT scan. The bright spot artifacts appear larger than the physical seed dimensions, so they do not accurately represent the patient geometry surrounding each source. These bright spot and streaking artifacts are caused by high attenuation in the metallic object resulting in a beam hardening effect and insufficient signal to the CT detectors. ${ }^{28}$ Earlier work has shown that the accuracy of dose calculations can be significantly diminished without first implementing a metallic artifact reduction algorithm to post-implant CT images. ${ }^{29-31}$ Artifact mitigation in CT images has been studied in the context of dental fillings ${ }^{28,32,33}$ and prosthesis, ${ }^{34-37}$ and several

general approaches including thresholding, ${ }^{29,30}$ spatial filters ${ }^{30,38}$ and sinogram space filters ${ }^{29-31,39}$ have been proposed. No published arifact mitigation method has been shown to work perfectly with the artifacts characteristic of brachytherapy images, and it remains unclear how differences in artifact mitigation approaches affect dose 
calculations.

Artifact corrected CT images are used to derive patient models by mapping each voxel's CT number to a mass density and elemental composition. CT number is represented in Hounsfield Units (HU) which are units of radiodensity defining water as $0 \mathrm{HU}$ and air as -1000 $\mathrm{HU}$. The maximum CT number range for a given $\mathrm{CT}$ scanner will depend on the model, but is often $2^{12}=4096 \mathrm{HU}$. CT number to mass density conversions are routinely obtained in the clinic, but often require an additional conversion of electron density to mass density. ${ }^{26,40}$ Defining tissue assignment mapping schemes (and dosimetric volumes of interest) typically relies on physician drawn contours, which are known to be highly subjective and inconsistent. ${ }^{41}$ Not only is the real distribution of tissues not well known due to the limited soft tissue contrast in $\mathrm{CT}$ images, the elemental composition used to calculate radiation interaction cross sections for each tissue varies across the literature ${ }^{42-46}$ and tissue composition has been shown to significantly affect dose calculations. ${ }^{47,48}$ Further, with the low energy photons from ${ }^{125} \mathrm{I}$ and ${ }^{103} \mathrm{Pd}$ sources, the photon interaction cross sections are highly dependent on the tissue effective atomic number, $\mathrm{Z}_{\text {eff }}$, since the predominate photon interaction mechanism, photoelectric effect, has a cross section proportional to at least $Z_{\text {eff }}^{3} \cdot{ }^{26,49}$ Thus, the development of well justified and widely adopted consensus patient modelling approaches are critical for consistency of practice for future adopters of MBDCAs for brachytherapy.

Several different types of MBDCAs exist, and currently two commercial MBDCA brachytherapy treatment planning and dose calculation software packages are available $;{ }^{27}$ Nucletron Oncentra Brachy which relies on a collapsed-cone superposition convolution ${ }^{50,51}$ and Varian Medical Systems BrachyVision which relies on a grid-based Boltzmann solver of the differential linear Boltzmann equation. ${ }^{52,53}$ An additional type of MBDCA, Monte Carlo simulations of radiation transport, is consid- 
ered the gold standard approach to calculate brachytherapy dose distributions, ${ }^{26}$ but has not yet been commercially adopted for brachytherapy as the more computationally efficient collapsed cone and Boltzmann equation solvers may provide comparable results within the range of acceptable clinical uncertainty. ${ }^{25}$ However, commercial Monte Carlo dose calculation packages are available for other radiation therapy applications which treat highly heterogeneous tissues with small fields, for example, the Accuray Cyberknife MultiPlan system. Recently ${ }^{27}$ the commercial brachytherapy MBDCAs were benchmarked against several Monte Carlo codes, and identified dose differences of up to $3 \%$ in basic water geometries with a high-energy ${ }^{192} \mathrm{Ir}$ source. It remains unclear how these commercial systems will compare to Monte Carlo dose calculations under more realistic heterogeneous geometries and with the low energy sources typical of permanent implant brachytherapy. Thus, there is still a need to pursue the development, and clinical adoption, of Monte Carlo dose calculations for permanent implant brachytherapy.

\subsection{Monte Carlo Dose Calculations}

Several non-commercial brachytherapy Monte Carlo dose calculation tools exist, for example, GEPTS based MCPI, ${ }^{54}$ GEANT4 based ALGEBRA ${ }^{55,56}$ and, the code which is used in this thesis, EGSnrc based BrachyDose. ${ }^{57,58}$ In 2000, the latest major revision of the Electron Gamma Shower (EGS) Monte Carlo code, EGSnrc, was released. ${ }^{59,60}$ EGSnrc is capable of simulating photon and electron transport, and score radiation dose, over a wide range of particle energies and virtual geometries. BrachyDose accurately models brachytherapy sources using combinations of basic geometric shapes ${ }^{61}$ and has been extensively benchmarked and used to generate TG-43 parameters for several commercial brachytherapy sources. ${ }^{4,57}$ BrachyDose $^{2}$ 
offers decreased calculation times relative to full radiation interaction simulations by estimating absorbed dose as collision kerma. The collision kerma approximation assumes radiative kerma (the energy lost to bremsstrahlung production) is zero, which is reasonable as $20 \mathrm{keV}$ electrons in soft tissue have a radiation yield of $0.00016 .^{62}$ Inherently, charged particle equilibrium is assumed, ${ }^{20}$ which is valid in brachytherapy treatments except very close to sources ${ }^{63}$ and near significant media heterogenieties such as metallic implants or bone. ${ }^{64}$ Additionally, BrachyDose can save computational time by not tracking electrons, and instead assuming their dose is deposited locally. This is a reasonable approximation as the CSDA range of a $20 \mathrm{keV}$ electron in $1 \mathrm{~g} / \mathrm{cm}^{3}$ soft tissue is $0.00086 \mathrm{~cm}^{62}$ and the voxel side-length in patient geometries are usually not smaller than $0.03 \mathrm{~cm}$. To score dose in a particular voxel, Brachydose uses a tracklength estimator, ${ }^{65}$

$$
D=K_{c o l}=\frac{\sum_{i} E_{i} T_{i}\left(\frac{\mu_{e n}}{\rho}\right)_{i}}{V}
$$

where $\mathrm{D}$ is dose, $\mathrm{K}_{\text {col }}$ is collision kerma, $\mathrm{E}_{i}$ is the $i$ th photon's energy, $\mathrm{T}_{i}$ is the $i$ th photon's track length through the voxel, $\left(\mu_{e n} / \rho\right)_{i}$ is the $i$ th photons mass energy absorption coefficient and $\mathrm{V}$ is the volume of the voxel. For statistical uncertainties of $2 \%$ in a $2 \times 2 \times 2 \mathrm{~mm}^{3}$ voxel mesh, BrachyDose calculation times have been reported to be on the order of 30 seconds. ${ }^{58}$

\subsection{Radiobiological Modelling}

Radiation dose is the classic quantity used to evaluate the quality of a radiation therapy treatment. However, the ultimate goal of radiotherapy is to kill the cancerous cells while inducing minimal unwanted side effects in healthy tissues. Several radiobiological models exist, including models specifically for permanent implant brachytherapy, 
which use radiation dose calculations to estimate biological dose and tumour control probabilities (see AAPM Task Group Report 137, and the references therein, for a comprehensive review $\left.{ }^{66}\right)$. Some of these models, which are still widely adopted to-

day, were first described upwards of 30 years ago ${ }^{67,68}$ and rely heavily on assumed biological parameters and mechanistic mathematical forms which have been shown to poorly fit clinical outcome data. ${ }^{69}$ It has not yet been shown how the differences in dose distributions calculated using full tissue $\mathrm{MC}$ dose calculations, compared to water-based TG-43 dose calculations, will affect these radiobiological models.

\subsection{Thesis Purpose}

This thesis has three purposes. First, to address outstanding challenges related to the development of accurate virtual patient models towards the clinical implementation of Monte Carlo dose calculations for permanent implant brachytherapy. Second, to explore these accurate Monte Carlo dose calculations in clinical data, and through comparisons with TG-43 water based dose calculations, develop a better understanding of the radiation dose distributions delivered to patients. Third, to apply these more accurate Monte Carlo dose calculations to existing radiobiological models for the first time to explore how differences in $\mathrm{MC}$ and clinical dose calculations may translate to differences in estimated biological effect.

\subsection{Thesis Outline}

Chapter 1 is an introduction to the scientific challenges addressed by this thesis. Chapter 2 describes the common MC dose calculation methodology used for the studies presented in subsequent Chapters. Chapter 3, Chapter 4 and Chapter 
5 investigate issues related to developing accurate virtual patient models in prostate and breast brachytherapy patients, and an example of this process is illustrated in Figure 1.2. Chapter 3 presents several metallic artifact mitigation methods to be applied to CT images before developing virtual patient models, and quantifies how differences between methods can affect dose calculations. Chapter 4 focuses on MC dose calculations in permanent implant breast brachytherapy, including issues related to artifact mitigation and assignment of tissue composition in patient models. Additionally, TG-43 and realistic tissue MC dose calculations are compared for the target volume and skin. Chapter 5 represents a large scale retrospective dosimetry study of 613 permanent implant prostate brachytherapy patients. Differences in dose calculations for the target volume and select organs-at-risk are explored between TG-43 and realistic tissue MC dose calculations. Additionally, a comprehensive dose calculation sensitivity analysis to tissue composition and calcification modelling approach is presented. Chapter $\mathbf{6}$ is an extension of the dosimetry study presented in Chapter 5, and applies the TG-43 and MC dose calculations to the most common permanent implant prostate brachytherapy radiobiological models. Differences in biological doses and expected tumour control probabilities between MC and TG-43, as well as between different radiobiological models is explored. Chapter 7 is a brief summary of the major conclusions of each Chapter, with remarks on outlook and potential future works. 


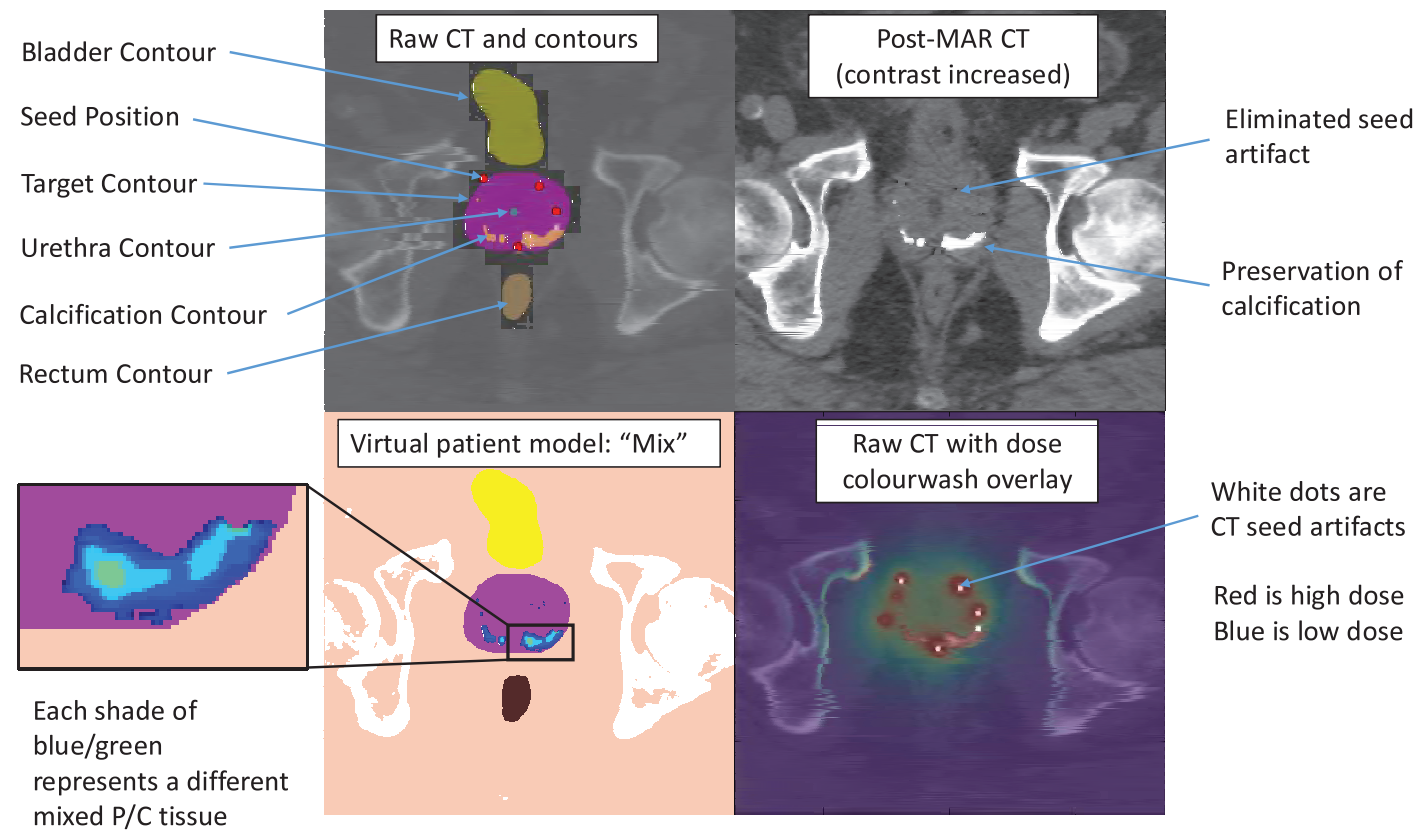

Figure 1.2: An example of the workflow to obtain a Monte Carlo dose calculation in a virtual patient model (this is a prostate patient). Top-Left, the raw (pre-MAR) CT image with an overlay of seed locations and physician drawn contours. Top-Right, the CT image after application of metallic artifact reduction (MAR) which eliminates the seed artifacts but preserves the calcification (MAR is studied in Chapter 3 and Chapter 4). Bottom-Left, the virtual patient model (example is the Mix model from Chapter 5) and a zoomed in region which shows the different mixed prostate $(\mathrm{P})$ and calcification (C) tissues used to model the calcification. Tissue assignment schemes and tissue compositions are studied in Chapter 3, Chapter 4 and Chapter 5. Bottom-Right, the calculated dose distribution overlayed onto the raw CT to illustrate the high dose near sources, and relatively higher doses scored in the calcification and the proximal edges of the pelvic bones. 


\section{Chapter 2}

\section{Monte Carlo Dose Calculation Methodology}

This Chapter describes the Monte Carlo (MC) dose calculation methods used throughout this thesis. This includes a brief description of the physics simulated by BrachyDose, the approach to develop virtual patent models from archived medical data and the process for analysing dose and statistical uncertainties. 


\subsection{BrachyDose Simulations}

BrachyDose, which relies on the underlying physics models of EGSnrc, ${ }^{70}$ simulates photon energies above $1 \mathrm{keV}$ by considering Rayleigh scattering, photoelectric absorption, Compton scattering and fluorescent emission of characteristic x-rays. BrachyDose relies on published photon cross sections which were obtained from the NIST XCOM database. ${ }^{71}$ Uncertainties on these cross sections may be on the order of $2 \%$ for most photon energies and more than $5 \%$ for energies below $5 \mathrm{keV} .{ }^{72}$ Assumed photon spectra are those published in TG-43. ${ }^{20}$ Mass energy absorption coefficients are calculated using EGSnrc user-code g. BrachyDose scores dose per simulated history, which is converted to absolute dose with a scaling factor,

$$
\text { ScalingFactor }=\frac{\tau S_{k}}{s_{k}},
$$

where $\tau$ is the mean lifetime of the radioisotope, $\mathrm{S}_{k}$ is the source air kerma strength at the time of treatment (specified by the vendor, and verified in the clinic), and $\mathrm{s}_{k}$ is the air-kerma strength per history for a particular source model which can be evaluated using Monte Carlo methods. ${ }^{57}$ More details on air-kerma strength can be found in TG-43. ${ }^{20}$

BrachyDose scores dose by simulating a large number of particles, also known as histories. Increasing the number of histories increases computational time, but lowers statistical uncertainty,

$$
T \propto \frac{1}{(\sigma)^{2}},
$$

where $\mathrm{T}$ is computational time and $\sigma$ is statistical uncertainty. Generally $10^{9}$ to $10^{11}$ histories were simulated for each dose calculation, typically resulting in sub 1\% (often sub $0.1 \%$ ) statistical uncertainty to target volume voxels, and requiring upwards of 24 hours of computational time across several CPUs per simulation. All simulations 
were performed on the Carleton University Research Compute Cluster which consists of 644 processing cores. More details on statistical uncertainty and calculation times for each study are provided in the appropriate Chapters.

BrachyDose offers additional options to decrease computational time. One may use Phase Space files which are pre-calculated particle fluences at the surface of sources, or one may use a run-mode called Particle Recycling which repeats a simulated particle which escaped a single source, over all other sources. For the work described in this thesis, no phase space files or particle recycling were used.

\subsection{Development of Virtual Patient Models}

Simulated patient geometries are defined in the egsphant file format, ${ }^{74}$ and derived from archived treatment data. The Digital Imaging and Communications in Medicine (DICOM) is the standard data model to store electronic radiology information, and its radiotherapy extension (DICOM-RT) is routinely used for brachytherapy. These DICOM-RT files contain post-implant CT images which can be used to derive a voxelized virtual patient model. The details of the mass density and tissue assignment schemes used to define virtual patient geometries are described in the relevant Chapters, but all tissue assignment is constrained by a voxel's location with respect to physician drawn contours (example, restrict assignment of prostate tissue to within the prostate contour). Elemental compositions of simulated tissues are derived from the literature and are common across Chapters (Tables 2.1,2.2). 
Table 2.1: Part1 Elemental compositions, effective atomic numbers, ${ }^{73} \mathrm{Z}_{\text {eff }}$, and mass densities of simulated tissues; $P$ and $C$ stand for prostate tissue and calcification, respectively (e.g., 80P20C refers to a homogeneous mixture of $80 \%$ Prostate and $20 \%$ Calcification(Breast) by mass). Composition proportions have been rounded so proportions may not add to exactly $100 \%$. The thesis Chapter, Ch., relying on each composition is identified.

\begin{tabular}{|c|c|c|c|c|c|c|c|c|}
\hline \multirow[b]{2}{*}{ Tissue } & \multicolumn{5}{|r|}{ Composition (Mass \%) } & \multirow{2}{*}{$\begin{array}{c}\rho \\
\left(\mathrm{g} / \mathrm{cm}^{3}\right)\end{array}$} & \multirow[b]{2}{*}{$\mathrm{Z}_{e f f}$} & \multirow[b]{2}{*}{$\mathrm{Ch}$} \\
\hline & $\mathrm{H}$ & $\mathrm{C}$ & $\mathrm{N}$ & $\mathrm{O}$ & Elements with $\mathrm{Z}>8$ & & & \\
\hline Mean Male Soft Tissue & 10.5 & 25.6 & 2.7 & 60.2 & $\mathrm{Na}(0.1), \mathrm{P}(0.2), \mathrm{S}(0.3), \mathrm{Cl}(0.2), \mathrm{K}(0.2)$ & 1.03 & 7.60 & 3,5 \\
\hline Prostate & 10.5 & 8.9 & 2.5 & 77.4 & $\mathrm{Na}(0.2), \mathrm{P}(0.1), \mathrm{S}(0.2), \mathrm{K}(0.2)$ & 1.04 & 7.77 & 3,5 \\
\hline $95 \mathrm{P} 5 \mathrm{C}$ & 10.0 & 8.5 & 2.4 & 75.6 & $\mathrm{Na}(0.2), \mathrm{P}(1.0), \mathrm{S}(0.2), \mathrm{K}(0.2), \mathrm{Ca}(1.9)$ & 1.08 & 8.86 & 3 \\
\hline $90 \mathrm{P} 10 \mathrm{C}$ & 9.5 & 8.2 & 2.3 & 73.7 & $\mathrm{Na}(0.2), \mathrm{P}(2.0), \mathrm{S}(0.2), \mathrm{K}(0.2), \mathrm{Ca}(3.8)$ & 1.11 & 9.71 & 3 \\
\hline $75 \mathrm{P} 25 \mathrm{C}$ & 8.0 & 7.1 & 2.0 & 68.2 & $\mathrm{Na}(0.2), \mathrm{P}(4.8), \mathrm{S}(0.2), \mathrm{K}(0.2), \mathrm{Ca}(9.6)$ & 1.25 & 11.53 & 3 \\
\hline 70P30C & 7.4 & 6.7 & 1.9 & 66.4 & $\mathrm{Na}(0.1), \mathrm{P}(5.7), \mathrm{S}(0.1), \mathrm{K}(0.1), \mathrm{Ca}(11.5)$ & 1.30 & 11.96 & 3 \\
\hline $65 \mathrm{P} 35 \mathrm{C}$ & 6.9 & 6.3 & 1.8 & 64.6 & $\mathrm{Na}(0.1), \mathrm{P}(6.6), \mathrm{S}(0.1), \mathrm{K}(0.1), \mathrm{Ca}(13.4)$ & 1.35 & 12.39 & 3 \\
\hline $60 \mathrm{P} 40 \mathrm{C}$ & 6.4 & 6.0 & 1.7 & 62.7 & $\mathrm{Na}(0.1), \mathrm{P}(7.5), \mathrm{S}(0.1), \mathrm{K}(0.1), \mathrm{Ca}(15.3)$ & 1.41 & 12.78 & 3,5 \\
\hline $55 \mathrm{P} 45 \mathrm{C}$ & 5.9 & 5.6 & 1.6 & 60.9 & $\mathrm{Na}(0.1), \mathrm{P}(8.5), \mathrm{S}(0.1), \mathrm{K}(0.1), \mathrm{Ca}(17.2)$ & 1.48 & 13.15 & 3 \\
\hline $50 \mathrm{P} 50 \mathrm{C}$ & 5.4 & 5.3 & 1.5 & 59.1 & $\mathrm{Na}(0.1), \mathrm{P}(9.4), \mathrm{S}(0.1), \mathrm{K}(0.1), \mathrm{Ca}(19.1)$ & 1.55 & 13.49 & 3,5 \\
\hline $25 \mathrm{P} 75 \mathrm{C}$ & 2.9 & 3.4 & 1.0 & 49.9 & $\mathrm{Na}(0.1), \mathrm{P}(14.1), \mathrm{S}(0.1), \mathrm{K}(0.1), \mathrm{Ca}(28.7)$ & 2.06 & 14.95 & 3 \\
\hline $20 \mathrm{P} 80 \mathrm{C}$ & 2.3 & 3.1 & 0.9 & 48.0 & $\mathrm{P}(15.0), \mathrm{Ca}(30.6)$ & 2.20 & 15.18 & 3,5 \\
\hline $15 \mathrm{P} 85 \mathrm{C}$ & 1.8 & 2.7 & 0.8 & 46.2 & $\mathrm{P}(15.9), \mathrm{Ca}(32.5)$ & 2.37 & 15.42 & 3 \\
\hline $10 \mathrm{P} 90 \mathrm{C}$ & 1.3 & 2.3 & 0.7 & 44.4 & $\mathrm{P}(16.8), \mathrm{Ca}(34.4)$ & 2.56 & 15.65 & 3 \\
\hline $5 \mathrm{P} 95 \mathrm{C}$ & 0.8 & 2.0 & 0.6 & 40.7 & $\mathrm{P}(17.8), \mathrm{Ca}(36.3)$ & 2.79 & 15.87 & 3 \\
\hline Calcification(Breast) & 0.3 & 1.6 & 0.5 & 40.7 & $\mathrm{P}(18.7), \mathrm{Ca}(38.2)$ & 3.06 & 16.09 & 3,5 \\
\hline Calcification-1 & 0.0 & 0.0 & 0.0 & 28.8 & $\mathrm{P}(23.1), \mathrm{S}(0.3), \mathrm{Cl}(0.4), \mathrm{K}(0.06), \mathrm{Ca}(39.6), \mathrm{Zn}(7.7), \mathrm{Sr}(0.04)$ & - & 18.89 & 5 \\
\hline
\end{tabular}


Table 2.2: Continuation of Table 2.1. $A$ and $G$ stand for adipose and gland tissue, respectively (e.g., 80A20G refers to a homogeneous mixture of $80 \%$ Adipose 2 and $20 \%$ Gland2 by mass).

\begin{tabular}{|c|c|c|c|c|c|c|c|c|}
\hline \multirow[b]{2}{*}{ Tissue } & \multicolumn{5}{|r|}{ Composition (Mass \%) } & \multirow{2}{*}{$\begin{array}{c}\rho \\
\left(\mathrm{g} / \mathrm{cm}^{3}\right)\end{array}$} & \multirow[b]{2}{*}{$\mathrm{Z}_{e f f}$} & \multirow[b]{2}{*}{ Ch. } \\
\hline & $\mathrm{H}$ & $\mathrm{C}$ & $\mathrm{N}$ & $\mathrm{O}$ & Elements with $\mathrm{Z}>8$ & & & \\
\hline Calcification-2 & 0.0 & 0.0 & 0.0 & 35.1 & $\mathrm{P}(24.9), \mathrm{S}(0.3), \mathrm{Cl}(0.2), \mathrm{K}(0.8), \mathrm{Ca}(35.6), \mathrm{Zn}(3.0), \mathrm{Sr}(0.03)$ & - & 17.26 & 5 \\
\hline Calcification-3 & 0.0 & 0.0 & 0.0 & 67.8 & $\mathrm{P}(1.1), \mathrm{S}(2.7), \mathrm{Cl}(0.7), \mathrm{K}(0.3), \mathrm{Ca}(27.1), \mathrm{Zn}(0.5)$ & - & 14.72 & 5 \\
\hline Calcification-4 & 0.0 & 0.0 & 0.0 & 45.9 & $\mathrm{P}(15.0), \mathrm{S}(3.2), \mathrm{Cl}(0.1), \mathrm{K}(0.6), \mathrm{Ca}(30.1) \mathrm{Zn}(5.0)$ & - & 17.29 & 5 \\
\hline Muscle & 10.2 & 14.3 & 3.4 & 71.0 & $\mathrm{Na}(0.1), \mathrm{P}(0.2), \mathrm{S}(0.3), \mathrm{Cl}(0.1) \mathrm{K}(0.4)$ & 1.05 & 7.85 & 5 \\
\hline Urinary Bladder(Full) & 10.8 & 3.5 & 1.5 & 83.0 & $\mathrm{Na}(0.3), \mathrm{P}(0.1), \mathrm{S}(0.1), \mathrm{Cl}(0.5), \mathrm{K}(0.2)$ & 1.03 & 7.98 & 5 \\
\hline Urinary Bladder(Empty) & 10.5 & 9.6 & 2.6 & 76.1 & $\mathrm{Na}(0.2), \mathrm{P}(0.2), \mathrm{S}(0.2), \mathrm{Cl}(0.3), \mathrm{K}(0.3)$ & 1.04 & 7.91 & 5 \\
\hline Urine & 11.0 & 0.5 & 1.0 & 86.2 & $\mathrm{Na}(0.4), \mathrm{P}(0.1), \mathrm{Cl}(0.6), \mathrm{K}(0.2)$ & 1.03 & 8.04 & 5 \\
\hline Rectum & 6.3 & 12.1 & 2.2 & 79.0 & $\mathrm{Na}(0.01), \mathrm{P}(0.1), \mathrm{Cl}(0.1), \mathrm{K}(0.1)$ & 0.75 & 7.74 & 5 \\
\hline GI Tract & 10.6 & 11.5 & 2.2 & 75.1 & $\mathrm{Na}(0.1), \mathrm{P}(0.1), \mathrm{S}(0.1), \mathrm{Cl}(0.2), \mathrm{K}(0.1)$ & 1.03 & 7.71 & 5 \\
\hline Air & 0.07 & 0.01 & 75.03 & 23.61 & $\operatorname{Ar}(1.27)$ & 0.001 & 7.24 & 4 \\
\hline Adipose1 & 11.2 & 51.7 & 1.3 & 35.5 & $\mathrm{Na}(0.1), \mathrm{S}(0.1), \mathrm{Cl}(0.1)$ & 0.97 & 6.85 & 4 \\
\hline Adipose2 & 11.4 & 59.8 & 0.7 & 27.8 & $\mathrm{Na}(0.1), \mathrm{S}(0.1), \mathrm{Cl}(0.1)$ & 0.95 & 6.67 & 4 \\
\hline Adipose3 & 11.6 & 68.1 & 0.2 & 19.8 & $\mathrm{Na}(0.1), \mathrm{S}(0.1), \mathrm{Cl}(0.1)$ & 0.93 & 6.47 & 4 \\
\hline AdiposeH & 11.2 & 61.9 & 1.7 & 25.1 & $\mathrm{P}(0.025), \mathrm{S}(0.025), \mathrm{K}(0.025), \mathrm{Ca}(0.025)$ & 0.93 & 6.58 & 4 \\
\hline Gland1 & 10.9 & 50.6 & 2.3 & 35.8 & $\mathrm{Na}(0.1), \mathrm{P}(0.1), \mathrm{S}(0.1), \mathrm{Cl}(0.1)$ & 0.99 & 6.90 & 4 \\
\hline Gland2 & 10.6 & 33.2 & 3.0 & 52.7 & $\mathrm{Na}(0.1), \mathrm{P}(0.1), \mathrm{S}(0.2), \mathrm{Cl}(0.1)$ & 1.02 & 7.27 & 4 \\
\hline Gland3 & 10.2 & 15.8 & 3.7 & 69.8 & $\mathrm{Na}(0.1), \mathrm{P}(0.1), \mathrm{S}(0.2), \mathrm{Cl}(0.1)$ & 1.06 & 7.80 & 4 \\
\hline GlandH & 10.2 & 18.4 & 3.2 & 67.7 & $\mathrm{P}(0.125), \mathrm{S}(0.125), \mathrm{K}(0.125), \mathrm{Ca}(0.125)$ & 1.04 & 7.63 & 4 \\
\hline $80 \mathrm{~A} 20 \mathrm{G}$ & 11.25 & 54.77 & 1.13 & 32.51 & $\mathrm{Na}(0.1), \mathrm{P}(0.02), \mathrm{S}(0.12), \mathrm{Cl}(0.1)$ & 0.96 & 6.80 & 4 \\
\hline Skin1 & 10.0 & 25.0 & 4.6 & 59.4 & $\mathrm{Na}(0.2), \mathrm{P}(0.1), \mathrm{S}(0.3), \mathrm{Cl}(0.3), \mathrm{K}(0.1)$ & 1.09 & 7.57 & 4 \\
\hline Skin2 & 10.0 & 20.4 & 4.2 & 64.5 & $\mathrm{Na}(0.2), \mathrm{P}(0.1), \mathrm{S}(0.2), \mathrm{Cl}(0.3), \mathrm{K}(0.1)$ & 1.09 & 7.63 & 4 \\
\hline Skin3 & 10.1 & 15.8 & 3.7 & 69.5 & $\mathrm{Na}(0.2), \mathrm{P}(0.1), \mathrm{S}(0.2), \mathrm{Cl}(0.3), \mathrm{K}(0.1)$ & 1.09 & 7.70 & 4 \\
\hline SkinH & 9.8 & 17.8 & 5.0 & 66.7 & $\mathrm{P}(0.175), \mathrm{S}(0.175), \mathrm{K}(0.175), \mathrm{Ca}(0.175)$ & 1.09 & 7.71 & 4 \\
\hline Lung & 10.3 & 10.5 & 3.1 & 74.9 & $\mathrm{Na}(0.2), \mathrm{P}(0.2), \mathrm{S}(0.3), \mathrm{Cl}(0.3), \mathrm{K}(0.2)$ & 1.05 & 7.88 & 4 \\
\hline
\end{tabular}




\subsection{Dose and Statistical Uncertainty Analysis}

BrachyDose returns a voxelized distribution of calculated doses and statistical uncertainties in the .3ddose file format. ${ }^{74}$ Dose volume histograms (DVHs) are constructed in 1 Gy bins using voxels with geometric centroids contained within each contoured volume. If multiple tissues exist within one contour, for example, prostate and calcification tissue within a CTV contour, then doses to all tissues are included in the DVH unless noted otherwise. DVH dose metrics (example $\mathrm{D}_{90}$ ) are extracted from DVHs using linear interpolation. Figure 2.1 is a visualization of how dose and volume metrics are defined from a DVH.

A history by history method is used to calculate statistical uncertainty in each voxel (described further in detail here ${ }^{75,76}$ ). The statistical uncertainty on a scored quantity $\mathrm{X}$ is,

$$
s_{\bar{X}}=\sqrt{\frac{1}{N-1}\left(\frac{\sum_{i=1}^{N} X_{i}^{2}}{N}-\left(\frac{\sum_{i=1}^{N} X_{i}}{N}\right)^{2}\right)},
$$

where $\mathrm{N}$ is the number of simulated histories. When describing uncertainty on dose metrics, the combined statistical uncertainty from the multiple voxels contributing to the DVH must be considered. Statistical uncertainties to volumes are evaluated as the quadrature sum of the voxel uncertainties within each contour considering only voxels which receive at least $25 \%$ of the prescription dose. ${ }^{26}$ This is a modification of the standard approach for calculating MBDCA uncertainties in external beam applications (brachytherapy uses 25\% instead of 50\%) to account for the sharper dose fall-off. ${ }^{77}$ 


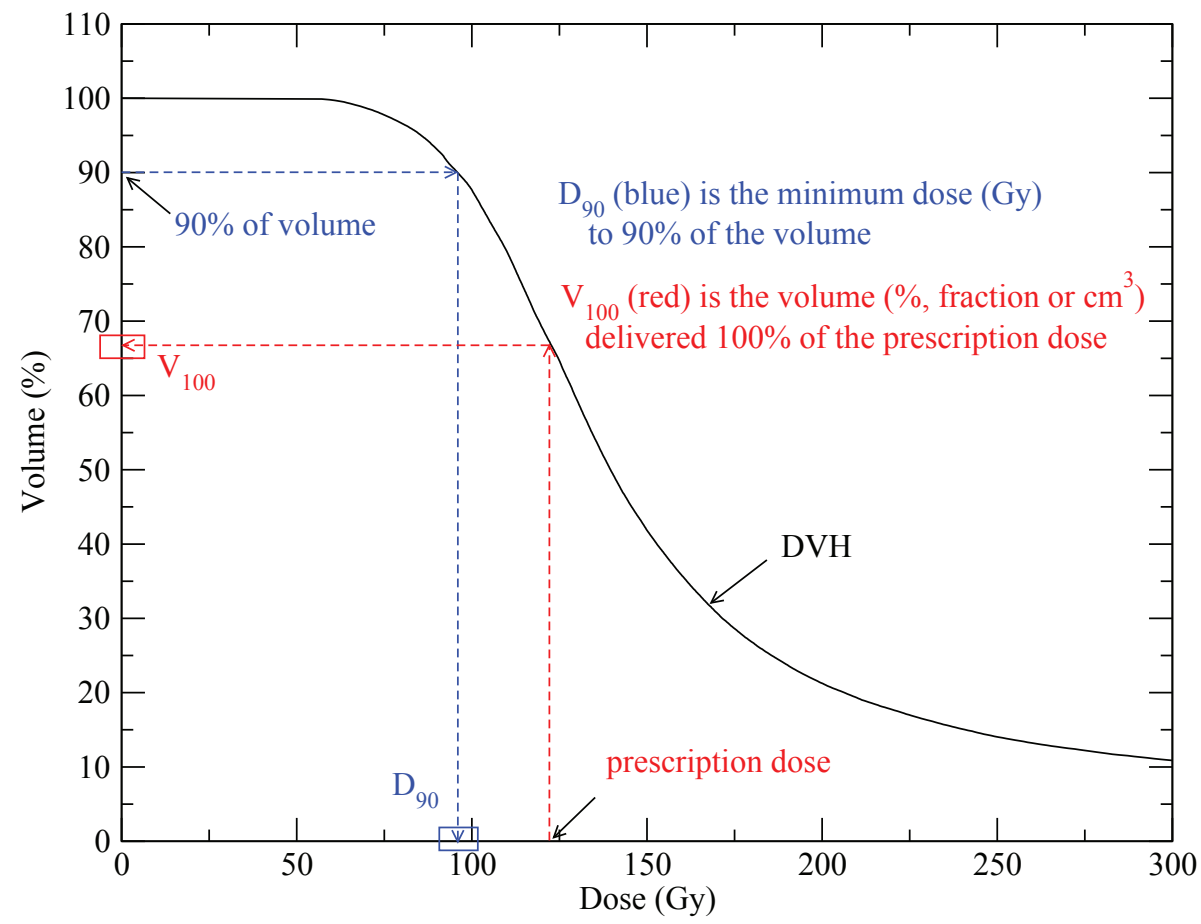

Figure 2.1: Visualization of how Dose Volume Histogram (DVH) dose metrics are defined, using $\mathrm{D}_{90}$ and $\mathrm{V}_{100}$ as examples. 


\section{Chapter 3}

\section{Metallic Artifact Reduction in CT}

\section{Images}

This Chapter explores metallic artifact reduction algorithms in a generalized prostate phantom and select prostate brachytherapy cases. The purpose of this study is to determine how the choice of artifact reduction method may affect dose calculations, and to determine which method(s) may be most suitable to implement for clinical brachytherapy Monte Carlo dose calculations. 


\subsection{Introduction}

Metallic Artifact Reduction (MAR) algorithms must be applied to post-implant CT images before deriving virtual patient models. No published MAR method eliminates all artifacts in all treatment sites and no comprehensive comparison of different MAR methods exists. It remains unclear how any missed or newly-introduced artifacts, which likely differ between MAR techniques, affect MC dose distributions.

MAR methods demonstrated in the literature are mainly for prostheses ${ }^{34-37}$ and dental ${ }^{28,32,33}$ applications; their extension to the brachytherapy context may be challenged by the large number of small and tightly spaced seeds, and the different anatomical sites. A few recent studies have begun to explore MAR methods for brachytherapy, demonstrating these techniques in idealized phantoms and a few se-

lect clinical cases: approaches include thresholding, ${ }^{29,30}$ image space filters, ${ }^{30,38}$ and projection space raw sinogram ${ }^{31,39}$ and virtual sinogram methods. ${ }^{29,30}$

In the prostate, uniformly-distributed calcifications have been shown to significantly decrease the target $\mathrm{D}_{90}$, by up to $37 \%$ in one study ${ }^{54}$ and by up to $10 \mathrm{~Gy}$ in another. ${ }^{78}$ Thus, accurate prostate brachytherapy dose calculations require the modelling of calcifications, however, both calcifications and metallic artifacts may correspond to very high CT numbers, thus challenging the mapping of CT number to tissue type and affecting computed doses. No study has investigated the impact on MC calculated doses from the interdependence of MAR method and tissue assignment modelling scheme, which is necessary before these methods can be applied to treatment sites possibly containing calcifications.

This Chapter investigates four computational MAR techniques to mitigate brachytherapy seed spot and streaking artifacts from post-implant CT images. Three image-based MAR methods (requiring only the reconstructed CT data) are consid- 
ered; a simple thresholding approach, a spatial 3D median filter and a virtual sinogram method, in addition to an approach using original scanner sinogram data. The MAR algorithms are first applied to a homogeneous brachytherapy phantom with implanted seeds where the underlying composition is known. This is to characterize the MAR methods by observing and comparing any characteristic missed and new artifacts. Then, the MAR methods are applied to eight clinical prostate brachytherapy cases to challenge them in more complex images where the true underlying tissue composition is unknown. Six different tissue assignment schemes of varying complexity are applied to the MAR-modified CT images to derive virtual patient models used in MC dose calculations. The MAR CT images, virtual patient models, and dose distributions are compared to explore the interrelation of metallic artifact mitigation and tissue assignment on local doses and clinical dose metrics for brachytherapy.

\subsection{Methods}

\subsubsection{Metallic Artifact Reduction}

Post-implant CT images of a generalized homogeneous brachytherapy phantom and eight clinical permanent implant prostate brachytherapy cases are considered. The phantom was constructed from six slabs of agarose gel and 70 implanted brachytherapy sources (decayed to background activity ${ }^{31}$ ); its CT image is 512 x 512 x 32 voxels of size $0.049 \mathrm{~cm} \mathrm{x} 0.049 \mathrm{~cm}$ x $0.25 \mathrm{~cm}$ (total image dimensions $25 \mathrm{~cm} \mathrm{x} 25 \mathrm{~cm} \mathrm{x}$ $8 \mathrm{~cm})$. The patient CT images are of the prostate and surrounding tissues, including

a clinically-defined CTV target contour prescribed 145 Gy using 45-74 implanted ${ }^{125} \mathrm{I}$ Amersham OncoSeed 6711 sources (Chapter 1 Figure 1.1) ; CT images are 512 x 512 x (75 to 81) voxels of size $0.033 \mathrm{~cm} \mathrm{x} 0.033 \mathrm{~cm} \mathrm{x} 0.25 \mathrm{~cm}$ (total image dimensions 
$16.9 \mathrm{~cm}$ x $16.9 \mathrm{~cm}$ x (18.8 to 20.3$) \mathrm{cm}$ ). CT images were previously acquired using a helical CT scanner (Somatom Emotion, Siemens Medical, Germany). The CT scans were acquired with an extended CT scale at $130 \mathrm{kV}$ and $150 \mathrm{~mA}$ with a $190 \mathrm{~mm}$ field of view and a helical pitch of $1.5 .^{31}$

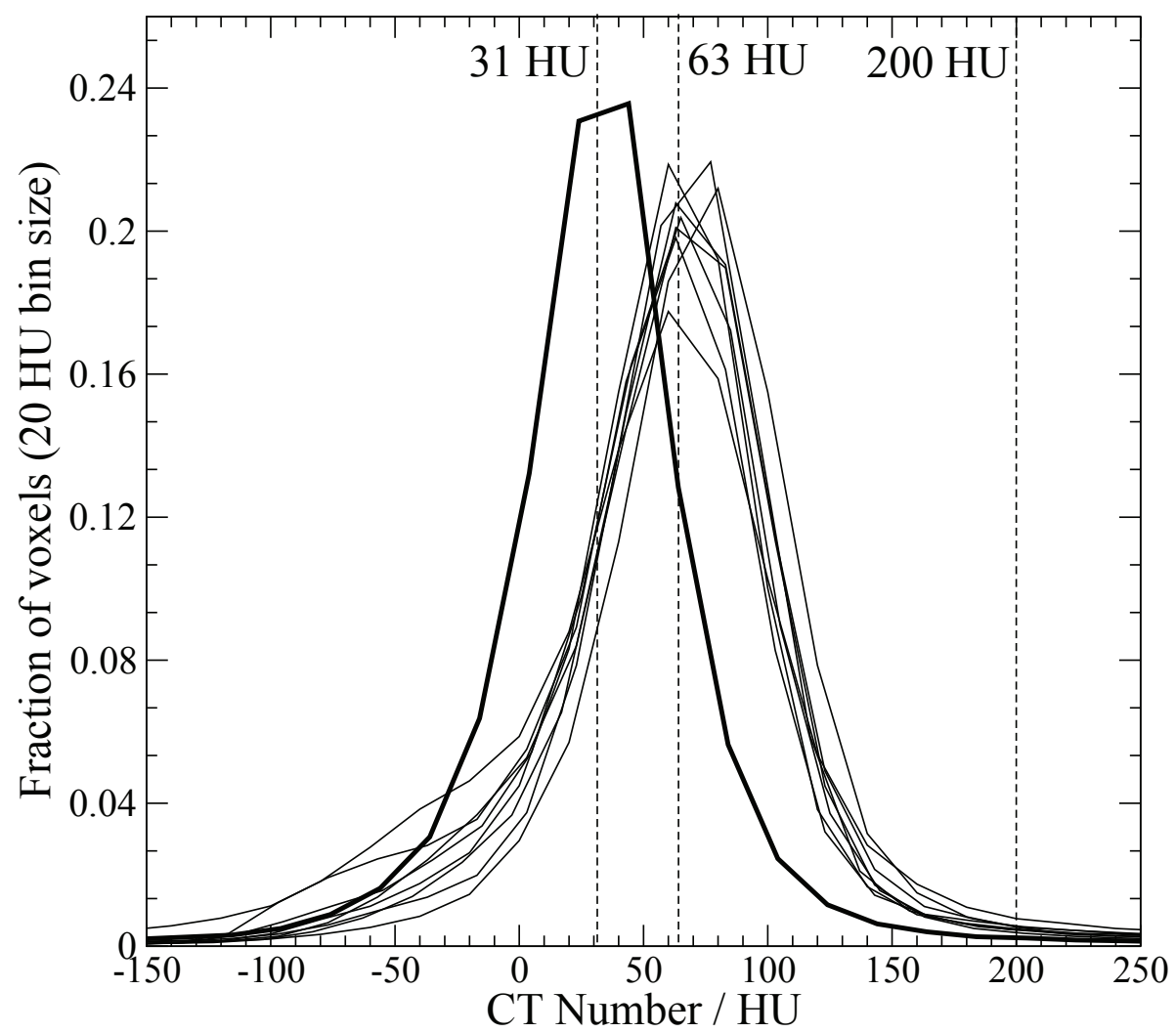

Figure 3.1: CT numbers of voxels within the homogeneous brachytherapy phantom (bold) and the CTV contour of the eight clinical prostate cases. Vertical dashed lines indicate values used for the MAR algorithms: mean CT number in the phantom (31 $\mathrm{HU})$, patient targets (63 HU), and the selected threshold value between soft tissue and artifact (200 HU).

Four metallic artifact reduction methods are compared: simple threshold replacement (STR), 3D median filter, fan beam virtual sinogram, and raw sinogram. The image-based methods (STR, median filter and virtual sinogram) require reconstructed post-implant $\mathrm{CT}$ images, unlike raw sinogram which requires the raw $\mathrm{CT}$ scanner sinogram data which is often not stored by institutions and may be in propri- 
etary file formats or inaccessible without vendor-granted permission. MAR methods are used to mitigate seed artifacts in addition to eliminating the high CT numbers corresponding to the seeds themselves from the images. Seed positions are defined in DICOM plan files and verified visually. All four MAR algorithms are written in MATLAB (The MathWorks, Inc., Natick, MA).

Simple threshold replacement (STR) uses thresholding to identify voxels corresponding to seeds and bright artifacts and replaces their CT numbers with a lower value. After parsing the seed centroid locations to the MAR algorithm, STR is constrained to voxels near seed positions to reduce the undesired elimination of high CT numbers representing bone or calcifications. Voxels are defined to be near seed positions if they are contained within cylindrical regions about the seed centres with a radius of $5 \mathrm{~mm}$ and a length of 5 slices (additional 2 slices in either direction from the slice containing the seed centre). The STR method uses a threshold of $200 \mathrm{HU}$ which is approximately the upper bound of the CT numbers corresponding to soft tissues (Figure 3.1). The replacement value is $31 \mathrm{HU}$ for the phantom and $63 \mathrm{HU}$ for prostate cases (the weighted mean of soft-tissue CT numbers in the 8 patient target contours) - see Figure 3.1.

The image-based 3D median filter is a two step method developed from a previous study ${ }^{38}$ and constrained to voxels near seed positions (as defined for STR) to avoid elimination of voxels corresponding to calcifications. In the first step, voxel values are compared with a threshold (200 HU): values above are replaced with a lower value (patients: $63 \mathrm{HU}$, phantom: $31 \mathrm{HU}$ ) whereas for values below, the median and standard deviation of CT numbers in a 3D local region about the voxel are evaluated (excluding values above the $200 \mathrm{HU}$ threshold). This 3D local region is defined as that within 5 voxels in a $\mathrm{CT}$ slice and within 3 adjacent slices above and below. The difference between the voxel value and the local median value is determined. If the 
difference is less than one quarter of the local standard deviation then the voxel value is unchanged, but if the difference exceeds this then the voxel value is replaced by the value of the voxel from an adjacent slice (above or below) which is closest to the calculated local median. The second filter smooths the image and differs from the first only in the last step where the voxel value is replaced with the local median value (and not the value from an adjacent slice) even if it is less than one quarter of the local standard deviation different than the median. The size of the local region in the second step was decreased (compared to the first step: 5 voxels to 3 voxels and 3 slices to 1 slice) to reduce over-smoothing which blurs tissue heterogenieties.

The fan beam virtual sinogram method builds on previous work ${ }^{47}$ but uses a fan beam transform instead of a parallel trace radon transform. The first step uses a threshold to delineate portions of the CT image which correspond to the seeds. Here, a high threshold of $1500 \mathrm{HU}$ was used to ensure the delineation includes only seeds and not the pelvic bones in the clinical CT images. Parts of the CT image below the threshold are assigned a value of zero, leaving a metal-only CT image, to which a fan beam transform is applied to generate a metal-only virtual sinogram. Next, the original, uncorrected CT image is applied a fan beam transform to construct a complete virtual sinogram. The metal-only sinogram is subtracted from the complete sinogram leaving a partial sinogram containing voids which are then filled with a cubic spline interpolation of the neighbouring pixels. Finally, an inverse fan beam transform is applied to the interpolated virtual sinogram to generate the artifact-mitigated CT image. The high threshold and cubic spline interpolation do not result in the complete removal of the artifacts immediately around the seed positions so a seed-position constrained STR with a threshold of $200 \mathrm{HU}$ is then applied. The MATLAB function fanbeam and ifanbeam are used with parameters FanRotationIncrement and FanSensorSpacing set to "0.1" and FanSensorGeometry set to "arc" and the fan beam vertex to centre of rotation parameter was set to 1000 
and 1400 for the phantom and clinical CT images, respectively. Where applicable, the fan beam parameter values were derived from the CT scanner geometry and then adjusted as necessary to minimise computational time while maintaining post-MAR CT image integrity.

The raw sinogram method has been previously shown to successfully mitigate metallic artifacts in the homogeneous phantom as well as one of the prostate

patient CT images used in this study. ${ }^{31}$ In this approach, components of the raw helical CT scanner sinogram data corresponding to the metallic sources are identified and replaced with an interpolation of surrounding data. The first step is to reconstruct the raw sinogram in a CT image using an inverse fan beam transform. Then, a multithresholding technique is used to identify the metal-only components of the image, which is then projected into a metal-only sinogram using a fan beam transform. The raw sinogram data is then applied a Steger detection algorithm to find the positions and boundaries of the seed traces. By combining the results of the raw sinogram Steger detection and the reconstructed CT image thresholding, the affected projections are identified, and replaced with an interpolation of unaffected neighbouring projections. Unlike the STR, 3D median filter and virtual sinogram MAR approaches, the raw sinogram method requires the original CT scanner sinogram data and scanner geometrical information for accurate reconstruction.

\subsubsection{Tissue Assignment}

A virtual patient model for dose calculations is derived from each CT image using a tissue assignment scheme which is a voxel-to-voxel mapping of CT number to tissue type and mass density. Six tissue assignment schemes are investigated (Table 3.1); all are contour-guided with different schemes for voxels inside and outside the target 
volume (physician-drawn CTV target contours retrieved from DICOM files). Table 2.1 in Chapter 2 gives elemental compositions for tissues.

The "TG-186" scheme follows baseline recommendations of TG-186: overwriting the density of all voxels with a uniform value, assigning tissue type based on voxel location with respect to image contours, and limiting the number of tissues to a few. ${ }^{26}$ Inside (outside) the target contour only prostate (mean male) tissue of the nominal mass density $1.04 \mathrm{~g} / \mathrm{cm}^{3}\left(1.03 \mathrm{~g} / \mathrm{cm}^{3}\right)$ is assigned. This approach produces patient models which are independent of MAR method and is used herein as a basis for comparison of dose distributions calculated using patient models produced with different MAR and tissue assignment schemes.

The "Simple-N" scheme is also motivated by the TG-186 approach of overwriting voxels affected by artifacts in the target, and assigns all target voxels to prostate of nominal (hence ' $\mathrm{N}$ ') density, $1.04 \mathrm{~g} / \mathrm{cm}^{3}$. However, outside the target, voxels are assigned to a mean male tissue or cortical bone with CT-derived density since bone is easily delineated from soft tissue and may improve the patient model by better accounting for realistic scattering conditions - this scheme for outside the target is used for all remaining tissue assignment schemes.

The remaining four schemes assign voxel mass densities within the target based on CT number (Table 3.2). The "Simple-CT" scheme assigns target voxels to prostate tissue. The "PC" scheme assigns prostate tissue and calcification in the target using the same $200 \mathrm{HU}$ threshold to differentiate the tissues as used in the MAR methods to identify high CT number artifacts. The "PC-20\%" scheme assigns prostate, calcification and homogeneous mixtures of prostate and calcification at $20 \%$ mass fraction increments (all with CT-derived mass density), allowing for modelling of lower density or diffuse micro-calcifications having CT numbers between those corresponding to the nominal values for prostate tissue and calcification. The CT 
Table 3.1: Six tissue assignment schemes ("TG-186", "Simple-N", "Simple-CT", "PC", "PC-20\%" and "PC-5\%") used to map voxel CT number to tissue type and mass density. Tissue assignment outside the target is the same for all schemes except "TG-186". Tissue compositions are defined in Chapter 2 Table 2.1; 'P' and 'C' stand for prostate tissue and calcification, respectively, and so, e.g., 95P5C refers to a homogeneous mixture of $95 \%$ prostate and $5 \%$ calcification by mass. Mass density is assigned either as a uniform literature nominal value, "Nominal", or derived from CT number, "CT", using the calibration curve in Table 3.2.

\begin{tabular}{|c|c|c|c|c|}
\hline Scheme name & Image region & Tissue & CT numbers & Density \\
\hline "TG-186" & $\begin{array}{l}\text { Outside Target } \\
\text { Inside Target }\end{array}$ & $\begin{array}{l}\text { Mean Male Tissue } \\
\text { Prostate }\end{array}$ & $\begin{array}{l}\text { All } \\
\text { All }\end{array}$ & $\begin{array}{l}\text { Nominal } \\
\text { Nominal }\end{array}$ \\
\hline All except "TG-186" & Outside Target & $\begin{array}{l}\text { Mean Male Tissue } \\
\text { Cortical Bone }\end{array}$ & $\begin{array}{l}\leq 200 \\
>200\end{array}$ & $\begin{array}{l}\mathrm{CT} \\
\mathrm{CT}\end{array}$ \\
\hline "Simple-N" & Inside Target & Prostate & All & Nominal \\
\hline "Simple-CT" & Inside Target & Prostate & All & $\mathrm{CT}$ \\
\hline "PC" & Inside Target & $\begin{array}{l}\text { Prostate } \\
\text { Calcification }\end{array}$ & $\begin{array}{l}\leq 200 \\
>200\end{array}$ & $\begin{array}{l}\mathrm{CT} \\
\mathrm{CT}\end{array}$ \\
\hline "PC-20\%" & Inside Target & $\begin{array}{l}\text { Prostate } \\
80 \mathrm{P} 20 \mathrm{C} \\
60 \mathrm{P} 40 \mathrm{C} \\
40 \mathrm{P} 60 \mathrm{C} \\
20 \mathrm{P} 80 \mathrm{C} \\
\text { Calcification }\end{array}$ & $\begin{array}{c}\leq 148 \\
149-495 \\
496-959 \\
960-1650 \\
1651-2792 \\
>2793\end{array}$ & $\begin{array}{l}\mathrm{CT} \\
\mathrm{CT} \\
\mathrm{CT} \\
\mathrm{CT} \\
\mathrm{CT} \\
\mathrm{CT}\end{array}$ \\
\hline "PC-5\%" & Inside Target & $\begin{array}{l}\text { Prostate } \\
\text { 95P5C } \\
\text { 90P10C } \\
\text { 85P15C } \\
\text { 80P20C } \\
75 \mathrm{P} 25 \mathrm{C} \\
\text { 70P30C } \\
65 \mathrm{P} 35 \mathrm{C} \\
60 \mathrm{P} 40 \mathrm{C} \\
\text { 55P45C } \\
\text { 50P50C } \\
\text { 45P55C } \\
\text { 40P60C } \\
\text { 35P65C } \\
\text { 30P70C } \\
\text { 25P75C } \\
\text { 20P80C } \\
\text { 15P85C } \\
\text { 10P90C } \\
\text { 5P95C } \\
\text { Calcification }\end{array}$ & $\begin{array}{c}\leq 45 \\
46-107 \\
108-190 \\
191-276 \\
277-358 \\
359-448 \\
449-545 \\
546-650 \\
651-765 \\
766-891 \\
892-1030 \\
1031-1184 \\
1185-1355 \\
1356-1546 \\
1547-1761 \\
1762-2006 \\
2007-2286 \\
2287-2610 \\
2611-2989 \\
2990-3437 \\
>3447 \\
\end{array}$ & $\begin{array}{l}\mathrm{CT} \\
\mathrm{CT} \\
\mathrm{CT} \\
\mathrm{CT} \\
\mathrm{CT} \\
\mathrm{CT} \\
\mathrm{CT} \\
\mathrm{CT} \\
\mathrm{CT} \\
\mathrm{CT} \\
\mathrm{CT} \\
\mathrm{CT} \\
\mathrm{CT} \\
\mathrm{CT} \\
\mathrm{CT} \\
\mathrm{CT} \\
\mathrm{CT} \\
\mathrm{CT} \\
\mathrm{CT} \\
\mathrm{CT} \\
\mathrm{CT}\end{array}$ \\
\hline
\end{tabular}


number boundary between each tissue is the midway point between the theoretical mass density of each homogeneous mixture based on the nominal densities of prostate and calcification $\left(1.04 \mathrm{~g} / \mathrm{cm}^{3}\right.$ and $3.06 \mathrm{~g} / \mathrm{cm}^{3}$, respectively). The "PC-5\%" model is based on the same idea as PC-20\%, however, it uses a finer grid of 5\% homogeneous mixed prostate and calcification increments.

For dose calculations within the agar phantom, the aforementioned (prostate) tissue assignment schemes are modified by replacing all prostate, mean male and cortical bone tissues with the composition of agarose gel, and the mixed prostate and calcification tissues with their equivalent mass fraction compositions of agarose gel mixed with calcification. The CT number boundaries between the mixed agar and calcification tissues are the same as in the "PC-5\%" and "PC-20\%" schemes (Table 3.1) since the observed mean CT number of the agar in the phantom (31 HU) matches the nominal density of prostate tissue $\left(1.04 \mathrm{~g} / \mathrm{cm}^{3}\right)$. A $6 \mathrm{~cm} \mathrm{x} 6 \mathrm{~cm} \mathrm{x} 4 \mathrm{~cm}$ box encompassing all the seeds is used as the CTV for tissue assignment and calculation of dose metrics.

Table 3.2: CT number to mass density calibration for the CT scanner used to acquire the images.

\begin{tabular}{cc}
\hline \hline CT number $(\mathrm{HU})$ & mass density $\left(\mathrm{g} / \mathrm{cm}^{3}\right)$ \\
\hline-832 & 0.217 \\
-522.8 & 0.508 \\
-74.2 & 0.967 \\
-34.7 & 0.99 \\
6.2 & 1.018 \\
47.8 & 1.061 \\
56.5 & 1.071 \\
244.2 & 1.159 \\
999 & 1.575 \\
\hline \hline
\end{tabular}




\subsubsection{Dose Calculations}

The EGSnrc user-code BrachyDose is used to calculate dose distributions in the voxelized virtual patient model with the same dose scoring grid as the CT image resolution. The number of histories (starting particles) for each simulation was $10^{10}$ resulting in an average statistical uncertainty to target voxels of less than $0.2 \%$. Dose to the local voxel medium is reported. Dose metrics presented are recommended by the American Brachytherapy Society: ${ }^{79} \mathrm{D}_{90}$ is the isodose surrounding $90 \%$ of the target volume and $\mathrm{V}_{100}\left(\mathrm{~V}_{150}\right)$ is the percentage of the target volume which receives $100 \%$ (150\%) of the 145 Gy prescription dose.

\subsection{Results}

\subsubsection{Metallic Artifact Reduction}

The phantom and patient post-implant CT images contain metallic artifacts appearing as bright spots surrounding the seeds and dark streaks emanating in several directions from each seed (examples: Figure 3.2 panels a and f). Profiles of CT number versus voxel position along the lines labelled 'A to B' and 'C to D' in Figure 3.2 (panels a, f) are provided in Figures 3.3 and 3.4 for the phantom and patient, respectively. Bright spot seed artifacts with peak CT numbers near $3100 \mathrm{HU}$ are several voxels (1-2 mm) in diameter. These artifacts may extend over several slices and artifacts on slices adjacent to the position of the seed centre may have lower CT numbers. The darkest portions of the streaks, found near the edges of the bright spots, are typically

not lower than $-300 \mathrm{HU}$ to $-400 \mathrm{HU}$ and gradually increase radially to the background CT number. Portions of the dark streaking artifacts which are not immediately next to the bright spots are usually between -50 and $50 \mathrm{HU}$, which is comparable to the 
noise range in the $\mathrm{CT}$ images.
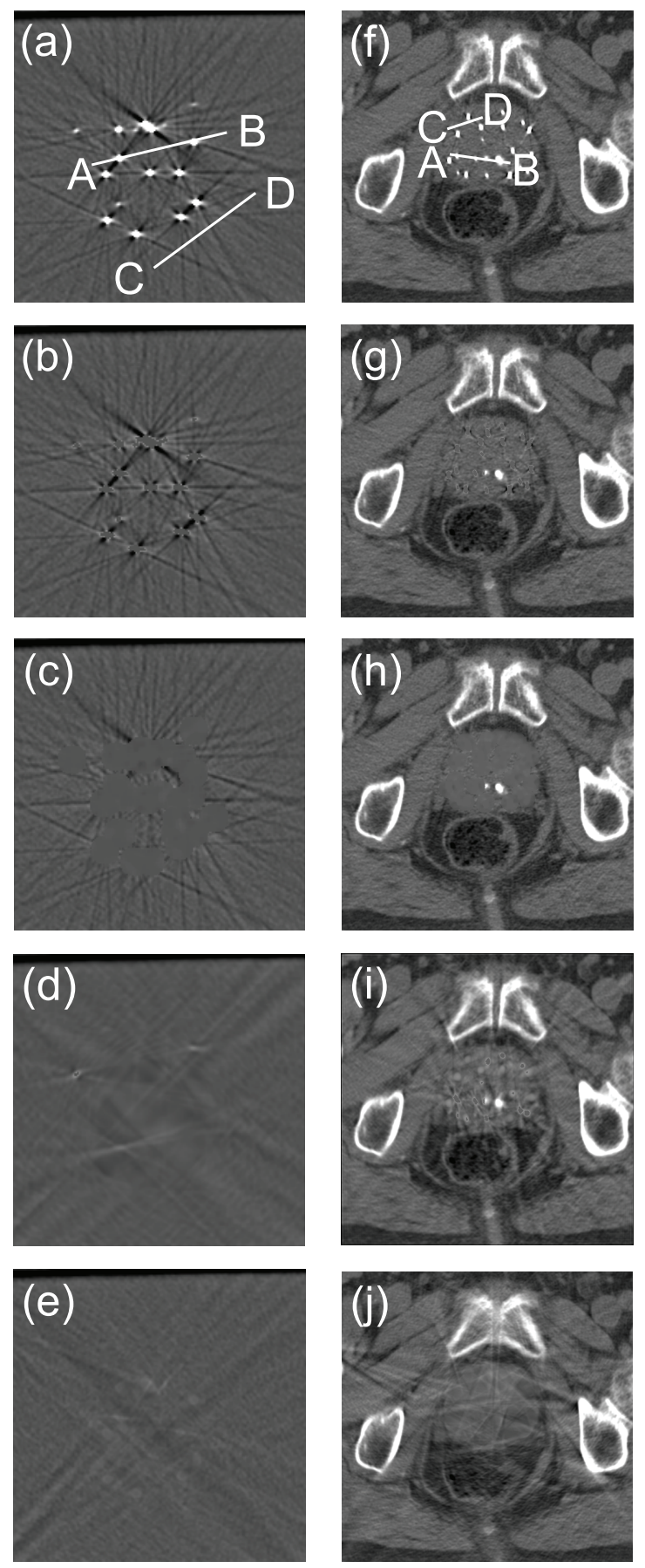

Figure 3.2: (a-e) Cropped slices of the homogeneous brachytherapy phantom and (f-j) axial slices of a prostate patient, illustrating the (a,f) uncorrected, (b,g) STR, (c,h) median filter, (d,i) virtual sinogram and $(e, j)$ raw sinogram CT images. Line profiles used in Figures 3.3, 3.4 and 3.5 are shown in panels (a) and (f). Images use a window level of $150 \mathrm{HU}$ and width of $700 \mathrm{HU}$ to emphasise artifacts and calcifications. 

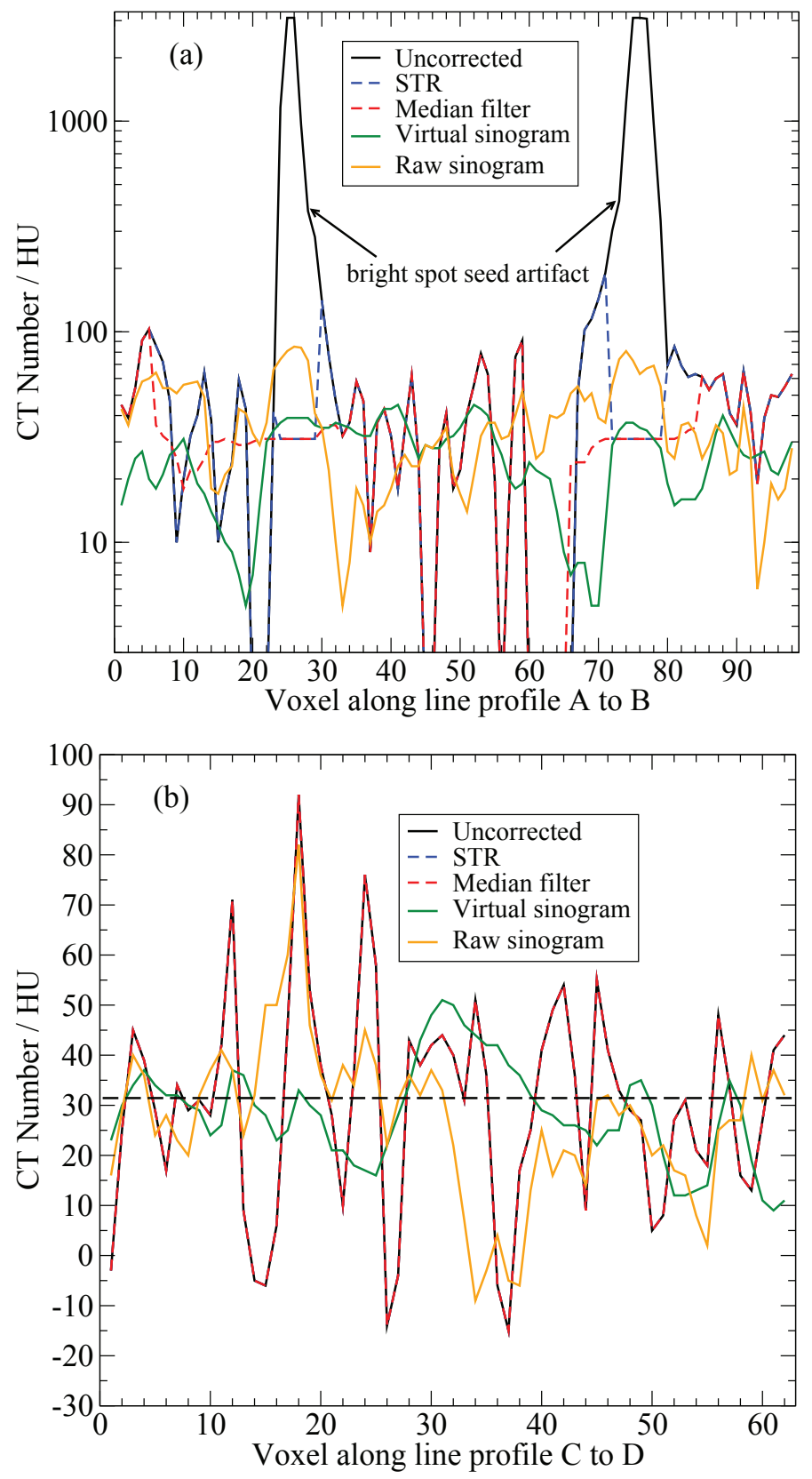

Figure 3.3: CT Numbers along lines (shown in Figure 3.2a), for a representative slice of the homogeneous brachytherapy phantom to compare the uncorrected, STR, median filter, virtual sinogram and raw sinogram images. (a) Bright seed spot artifacts along line profile A to B. (b) Streaking artifacts and image noise along line profile C to D. Uncorrected, STR and median filter are coincident lines. The horizontal dashed line represents the mean CT number (31 HU) in the phantom. 

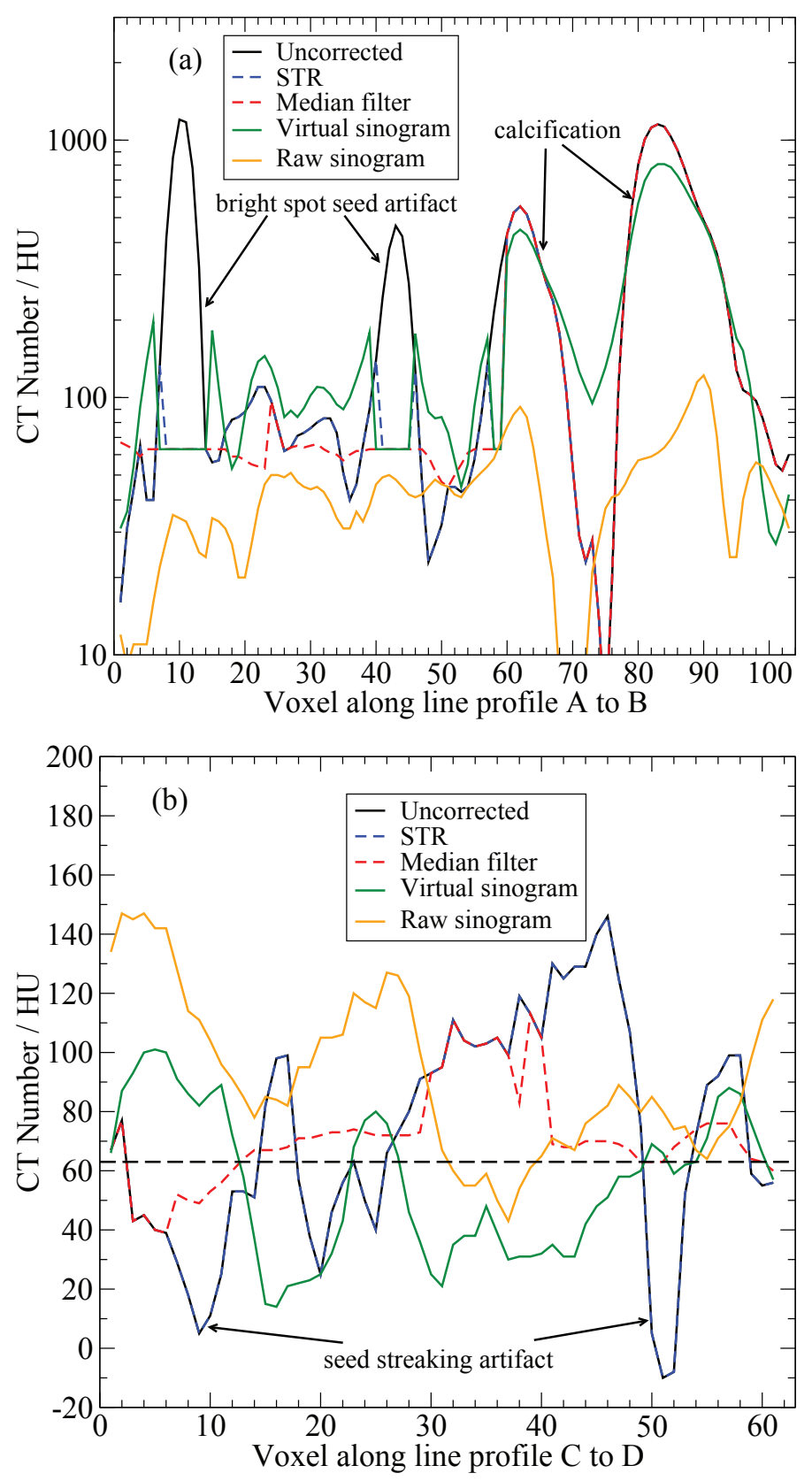

Figure 3.4: CT Numbers along lines (shown in Figure 3.2f), for a representative slice of one prostate patient to compare the uncorrected, STR, median filter, virtual sinogram and raw sinogram images. (a) Bright seed spot artifact and calcification along line profile A to B. (b) Streaking artifacts and image noise along line profile C to D. Uncorrected and STR are coincident lines. The horizontal dashed line represents the mean CT number (63 HU) in the target volumes of the prostate patients.

In the CT images which have STR applied, the bright spot artifacts around 
each seed are removed and replaced with a single CT value (figure 3.2, panels b and g). The STR method is unable to mitigate any dark streaking artifacts (by virtue of its design). The median filter shows a blurring effect, but successful mitigation of bright and dark artifacts in regions around the seeds where it was applied (Figure 3.2, panels $\mathrm{c}$ and $\mathrm{h}$ ). The virtual sinogram (Figure 3.2, panels $\mathrm{d}$ and i) and the raw sinogram (Figure 3.2, panels e and $\mathrm{j}$ ) mitigate the bright and dark seed artifacts; however, both methods introduce new streaks which are much less pronounced than the original streaks from the seeds and are generally comparable to the typical variations in background CT number. The new streaks are more apparent in the prostate cases than for the phantom and appear to originate from the edges of the bones. The extensiveness of the new streaks differs from patient to patient, and even slice to slice within the same patient, but the examples shown in Figure 3.2 are typical. The CT numbers of each post-MAR image along the line profiles defined in Figure 3.2 (panels $\mathrm{a}$ and f) can be seen in Figures 3.3 and 3.4.

None of the MAR methods are found to preserve all calcifications, and the calcifications which are eliminated are not consistent between MAR methods. For example, Figure 3.4a shows calcifications preserved with STR and median filter, completely eliminated by the raw sinogram, and partially eliminated by the virtual sinogram. All MAR methods are challenged when calcifications are close to seed positions.

CT number mean and standard deviation within square regions of interest (ROI) are investigated over the treatment volume where artifacts are abundant, "near ROI", and in distal regions of images where artifacts are sparse, "far ROI" - Table 3.3 provides some representative results (from the many cases considered). For both the phantom and patient cases, the mean and standard deviations of CT numbers in the "near ROI" are higher for the uncorrected images than for the post-MAR images 
because of the high CT number artifacts. Comparing post-MAR images, mean CT numbers in the "near-ROI" vary slightly with MAR method; standard deviations are higher for STR than for the other MAR methods due to missed dark artifacts while for median filter the deviations are slightly lower due to the blurring (averaging). For the "far ROI", STR and median filter have the same mean and standard deviation CT numbers as the uncorrected images because these MAR methods are constrained to seed positions and not applied in the distal regions of the CT image; the means and standard deviations for the two sinogram methods are comparable despite the introduction of new streaks.

Table 3.3: Example mean $(\bar{x})$ and standard deviation $(\sigma)$ of CT numbers (HU) within regions of interest in the phantom and two patients in uncorrected and MAR CT images. Regions of interest are defined as squares within one slice, near seeds ("near ROI") and away from seeds near the edge of the image ("far ROI").

\begin{tabular}{|c|c|c|c|c|c|c|c|c|c|c|c|c|}
\hline \multirow[b]{3}{*}{ MAR image } & \multicolumn{4}{|c|}{ Phantom } & \multicolumn{4}{|c|}{ Patient 1} & \multicolumn{4}{|c|}{ Patient 2} \\
\hline & \multicolumn{2}{|c|}{ near ROI } & \multicolumn{2}{|c|}{ far ROI } & \multicolumn{2}{|c|}{ near ROI } & \multicolumn{2}{|c|}{ far ROI } & \multicolumn{2}{|c|}{ near ROI } & \multicolumn{2}{|c|}{ far ROI } \\
\hline & $\bar{x}$ & $\sigma$ & $\bar{x}$ & $\sigma$ & $\bar{x}$ & $\sigma$ & $\bar{x}$ & $\sigma$ & $\bar{x}$ & $\sigma$ & $\bar{x}$ & $\sigma$ \\
\hline Uncorrected & 68 & 306 & 35 & 10 & 99 & 256 & 48 & 52 & 118 & 300 & 60 & 29 \\
\hline STR & 26 & 51 & 35 & 10 & 61 & 42 & 48 & 52 & 65 & 73 & 60 & 29 \\
\hline Median filter & 31 & 22 & 35 & 10 & 61 & 25 & 48 & 52 & 65 & 64 & 60 & 29 \\
\hline Virtual sinogram & 29 & 15 & 34 & 8 & 68 & 39 & 47 & 54 & 75 & 67 & 64 & 33 \\
\hline Raw sinogram & 32 & 16 & 34 & 9 & 65 & 35 & 50 & 52 & 55 & 35 & 66 & 30 \\
\hline
\end{tabular}

\subsubsection{Tissue Assignment}

The number of virtual patient model voxels assigned to each tissue type depends on the type of MAR due to differences in post-MAR CT numbers. Table 3.4 shows the number of CTV voxels assigned to each tissue after application of the "PC", "PC-20\%" and "PC-5\%" tissue assignment schemes (Table 3.1) in the homogeneous phantom and two example patient cases: a patient with no visible calcifications $(\# 1)$ and another with visible large and diffuse sub-voxel sized calcifications (\#2), but 
Table 3.4: The percentage of virtual patient model voxels assigned to each tissue using the "PC", "PC-20\%", and "PC-5\%" tissue assignment schemes with uncorrected (Unc) or MAR (STR, median filter (Med), virtual sinogram (VS) and raw sinogram $(\mathrm{RS})$ ) CT images for the brachytherapy phantom and two patient cases. "Patient 1" had no visible calcifications, while "Patient 2" had several visible calcifications and diffuse micro-calcified regions.

\begin{tabular}{|c|c|c|c|c|c|c|c|c|c|c|c|c|c|c|c|c|}
\hline \multirow[t]{2}{*}{$\overline{T A S}$} & \multirow[b]{2}{*}{ Tissue } & \multicolumn{5}{|c|}{ Phantom } & \multicolumn{5}{|c|}{ Patient 1} & \multicolumn{5}{|c|}{$\overline{\text { Patient } 2}$} \\
\hline & & Unc & STR & Med & VS & $\overline{\mathrm{RS}}$ & Unc & STR & Med & VS & $\overline{\mathrm{RS}}$ & Unc & STR & Med & $\overline{V S}$ & $\overline{\mathrm{RS}}$ \\
\hline \multirow[t]{2}{*}{$\mathrm{PC}$} & Prostate & 96.94 & 100 & 100 & 100 & 99.76 & 92.69 & 99.93 & 99.93 & 99.93 & 99.92 & 92.03 & 99.50 & 99.50 & 99.19 & 99.55 \\
\hline & Calcification & 3.06 & 0.00 & 0.00 & 0.00 & 0.24 & 7.31 & 0.07 & 0.07 & 0.07 & 0.08 & 7.97 & 0.50 & 0.50 & 0.81 & 0.45 \\
\hline \multirow[t]{6}{*}{ PC-20\% } & Prostate & 96.03 & 99.10 & 99.97 & 99.79 & 99.44 & 89.85 & 97.12 & 99.69 & 94.04 & 98.65 & 89.68 & 97.18 & 99.25 & 93.63 & 98.07 \\
\hline & $80 \%$ p $20 \%$ c & 2.26 & 0.90 & 0.03 & 0.21 & 0.52 & 6.44 & 2.88 & 0.31 & 5.96 & 1.35 & 6.03 & 2.67 & 0.60 & 6.26 & 1.93 \\
\hline & $60 \%$ p $40 \%$ c & 0.54 & 0.00 & 0.00 & 0.00 & 0.03 & 1.63 & 0.00 & 0.00 & 0.00 & 0.00 & 1.99 & 0.13 & 0.13 & 0.11 & 0.00 \\
\hline & $40 \%$ p $60 \%$ c & 0.39 & 0.00 & 0.00 & 0.00 & 0.01 & 0.92 & 0.00 & 0.00 & 0.00 & 0.00 & 1.09 & 0.02 & 0.02 & 0.00 & 0.00 \\
\hline & $20 \%$ p $80 \%$ c & 0.33 & 0.00 & 0.00 & 0.00 & 0.00 & 0.69 & 0.00 & 0.00 & 0.00 & 0.00 & 0.65 & 0.00 & 0.00 & 0.00 & 0.00 \\
\hline & Calcification & 0.45 & 0.00 & 0.00 & 0.00 & 0.00 & 0.46 & 0.00 & 0.00 & 0.00 & 0.00 & 0.56 & 0.00 & 0.00 & 0.00 & 0.00 \\
\hline \multirow[t]{21}{*}{ PC- $5 \%$} & Prostate & 63.89 & 66.96 & 82.38 & 74.42 & 72.28 & 24.20 & 24.20 & 10.16 & 19.38 & 24.11 & 22.63 & 22.63 & 6.19 & 19.15 & 21.42 \\
\hline & $95 \%$ p $5 \%$ c & 29.82 & 29.82 & 17.05 & 24.57 & 26.00 & 55.64 & 62.91 & 86.35 & 60.61 & 63.70 & 58.92 & 66.42 & 91.08 & 61.05 & 66.25 \\
\hline & $90 \%$ p $10 \%$ c & 3.10 & 3.10 & 0.57 & 0.99 & 1.43 & 12.52 & 12.52 & 3.41 & 19.16 & 12.07 & 10.16 & 10.16 & 2.20 & 18.31 & 11.77 \\
\hline & $85 \%$ p $15 \%$ c & 0.75 & 0.12 & 0.00 & 0.02 & 0.16 & 1.98 & 0.34 & 0.06 & 0.82 & 0.11 & 1.86 & 0.45 & 0.19 & 1.05 & 0.49 \\
\hline & $80 \%$ p $20 \%$ c & 0.37 & 0.00 & 0.00 & 0.00 & 0.05 & 1.01 & 0.02 & 0.02 & 0.02 & 0.00 & 1.02 & 0.09 & 0.09 & 0.18 & 0.06 \\
\hline & $75 \%$ p $25 \%$ c & 0.26 & 0.00 & 0.00 & 0.00 & 0.03 & 0.69 & 0.01 & 0.01 & 0.01 & 0.00 & 0.79 & 0.06 & 0.06 & 0.12 & 0.01 \\
\hline & $70 \%$ p $30 \%$ c & 0.18 & 0.00 & 0.00 & 0.00 & 0.02 & 0.55 & 0.00 & 0.00 & 0.00 & 0.00 & 0.63 & 0.06 & 0.06 & 0.07 & 0.00 \\
\hline & $65 \%$ p $35 \%$ c & 0.15 & 0.00 & 0.00 & 0.00 & 0.01 & 0.44 & 0.00 & 0.00 & 0.00 & 0.00 & 0.55 & 0.04 & 0.04 & 0.04 & 0.00 \\
\hline & $60 \%$ p $40 \%$ c & 0.13 & 0.00 & 0.00 & 0.00 & 0.00 & 0.41 & 0.00 & 0.00 & 0.00 & 0.00 & 0.48 & 0.03 & 0.03 & 0.03 & 0.00 \\
\hline & $55 \%$ p $45 \%$ c & 0.13 & 0.00 & 0.00 & 0.00 & 0.00 & 0.34 & 0.00 & 0.00 & 0.00 & 0.00 & 0.46 & 0.02 & 0.02 & 0.01 & 0.00 \\
\hline & $50 \%$ p $50 \%$ c & 0.12 & 0.00 & 0.00 & 0.00 & 0.01 & 0.28 & 0.00 & 0.00 & 0.00 & 0.00 & 0.38 & 0.02 & 0.02 & 0.00 & 0.00 \\
\hline & $45 \%$ p $55 \%$ c & 0.10 & 0.00 & 0.00 & 0.00 & 0.00 & 0.25 & 0.00 & 0.00 & 0.00 & 0.00 & 0.33 & 0.01 & 0.01 & 0.00 & 0.00 \\
\hline & $40 \%$ p $60 \%$ c & 0.10 & 0.00 & 0.00 & 0.00 & 0.00 & 0.23 & 0.00 & 0.00 & 0.00 & 0.00 & 0.28 & 0.00 & 0.00 & 0.00 & 0.00 \\
\hline & $35 \%$ p $65 \%$ c & 0.09 & 0.00 & 0.00 & 0.00 & 0.00 & 0.21 & 0.00 & 0.00 & 0.00 & 0.00 & 0.21 & 0.00 & 0.00 & 0.00 & 0.00 \\
\hline & $30 \%$ p $70 \%$ c & 0.08 & 0.00 & 0.00 & 0.00 & 0.00 & 0.19 & 0.00 & 0.00 & 0.00 & 0.00 & 0.18 & 0.00 & 0.00 & 0.00 & 0.00 \\
\hline & $25 \%$ p $75 \%$ c & 0.08 & 0.00 & 0.00 & 0.00 & 0.00 & 0.18 & 0.00 & 0.00 & 0.00 & 0.00 & 0.17 & 0.00 & 0.00 & 0.00 & 0.00 \\
\hline & $20 \%$ p $80 \%$ c & 0.08 & 0.00 & 0.00 & 0.00 & 0.00 & 0.17 & 0.00 & 0.00 & 0.00 & 0.00 & 0.18 & 0.00 & 0.00 & 0.00 & 0.00 \\
\hline & $15 \%$ p $85 \%$ c & 0.08 & 0.00 & 0.00 & 0.00 & 0.00 & 0.17 & 0.00 & 0.00 & 0.00 & 0.00 & 0.15 & 0.00 & 0.00 & 0.00 & 0.00 \\
\hline & $10 \%$ p $90 \%$ c & 0.08 & 0.00 & 0.00 & 0.00 & 0.00 & 0.16 & 0.00 & 0.00 & 0.00 & 0.00 & 0.16 & 0.00 & 0.00 & 0.00 & 0.00 \\
\hline & $5 \%$ p $95 \%$ c & 0.41 & 0.00 & 0.00 & 0.00 & 0.00 & 0.38 & 0.00 & 0.00 & 0.00 & 0.00 & 0.47 & 0.00 & 0.00 & 0.00 & 0.00 \\
\hline & Calcification & 0.00 & 0.00 & 0.00 & 0.00 & 0.00 & 0.00 & 0.00 & 0.00 & 0.00 & 0.00 & 0.00 & 0.00 & 0.00 & 0.00 & 0.00 \\
\hline
\end{tabular}


corresponding to less than $1 \%$ of the voxels in the CTV. In all cases, uncorrected CT images lead to a larger fraction of voxels assigned to calcification (or calcification mixture), compared to MAR images, due to the high CT number seed artifacts.

In the homogeneous phantom, the relative fraction of voxels assigned to each tissue type is the same using the PC scheme and the modified PC scheme (used for dose calculation in the homogeneous phantom) which replaces prostate tissue with agarose, since the CT number boundaries are the same. Applying the PC scheme to homogeneous phantom MAR images results in all CTV voxels assigned to prostate, except for raw sinogram which contained some high CT number streaks that were assigned to calcification. Applying the PC-20\% scheme to the phantom MAR images leads to $>99 \%$ of voxels assigned to prostate, with the other voxels corresponding to either bright streaking artifacts which are far enough from the seeds to be missed by the MAR or voxels with CT numbers below the threshold used in MAR (200 HU) and above the upper CT number boundary of prostate (148 HU) in this tissue assignment scheme. Alternate MAR images generated using a $148 \mathrm{HU}$ threshold were explored (results not reported) leading to no more than $0.03 \%$ of voxels assigned to mixed tissue for the PC-20\% scheme with STR, median filter, and virtual sinogram. Applying the "PC-5\%" scheme to the phantom MAR images results in most voxels assigned to prostate, but also a considerable fraction assigned to mixed tissue since the upper end of the distribution of CT numbers in the CTV falls above the upper CT number boundary for prostate (45 HU) in the tissue assignment scheme.

For the clinical cases, the "PC-5\%" scheme assigns more voxels to $95 \%$ prostate and 5\% calcification than pure prostate since the mean CT number in the target volumes (average for all patients) corresponds to a mass density of $1.07 \mathrm{~g} / \mathrm{cm}^{3}$ which falls above the upper CT number threshold for prostate. Generally, the CT number of calcifications is much lower (corresponding to a density of $1.75 \mathrm{~g} / \mathrm{cm}^{3}$ or 
lower) than that associated with the nominal literature density, $3.06 \mathrm{~g} / \mathrm{cm}^{3}$. As a result, even large calcifications are assigned to mixtures of prostate and calcification in the "PC-5\%" and "PC-20\%" schemes. The median filter draws more CT numbers towards the average value which, in the patient cases, results in more voxels assigned to prostate for the "PC-20\%" scheme and to $95 \%$ prostate/5\% calcification for the "PC-5\%" scheme compared to the other MAR images. The new streaks introduced by the virtual and raw sinogram MAR methods result in more voxels assigned to mixed tissue with higher calcification content compared to patient models derived using the other MAR images.

\subsubsection{Dose Distributions}

In the agar phantom, dose metrics for MC models with various MAR and tissue assignment schemes that assign density based on CT number are on average $2 \%$ lower (2.5\% standard deviation) than the basic "TG-186" scheme which assigns uniform nominal density tissue (Table 3.5). The spread in metrics between different MAR methods and tissue assignment schemes suggest a baseline level of uncertainty of several percent introduced by the differences in, and interdependence of, MAR methods and tissue assignment schemes.

Table 3.6 provides dose metrics for the patients computed with the "TG-186" scheme for which results are independent of MAR method, while Table 3.7 summarizes dose metrics for different MARs and tissue assignment schemes relative to "TG-186". In patients, dose metrics calculated using uncorrected CT images and the "SimpleN" scheme are up to $11 \%$ lower for $\mathrm{D}_{90}$ and $\mathrm{V}_{150}$ compared to those calculated using the "TG-186" scheme. While both the "TG-186" and "Simple-N" schemes assign uniform density prostate tissue within the CTV (over-writing artifacts), the "Simple- 
N" scheme maps seed artifacts outside the target to cortical bone causing high doses calculated near these seeds and lower doses to soft tissues due to the reduced fluence (Figure 3.5a). Thus, the MAR and tissue assignment approaches must take into account the fact that sources may be located outside the target volume.

Compared to "Simple-N", virtual patient models derived using "Simple-CT" applied to the uncorrected images have lower dose metrics by a few percent as a result of higher density prostate tissue being mapped to artifacts around the seeds in the target. Applying any of the schemes which model calcification in the prostate ("PC", "PC-20\%" and "PC-5\%") to the uncorrected images results in encapsulation of all seeds in bone (outside target) or calcification (inside target) resulting in low dose metrics. The reduction in metrics with "PC-20\%" and "PC-5\%" is less significant than with "PC" because higher CT number voxels are mapped to mixed prostate-calcification tissue rather than to pure calcification. Figure 3.6 illustrates the importance of reasonable artifact mitigation and tissue assignment by providing ratios of doses for uncorrected (a) and STR (b) patient models applied the "PC" tissue assignment scheme relative to the TG-186 scheme in a plane: there are considerable dose reductions due to seeds modelled as encased in calcification (Figure $3.6 a)$. 
Table 3.5: Summary of homogeneous agar phantom dose metrics normalized to "TG186". Dose distributions are calculated in patient models derived from uncorrected and MAR CT images using one of three tissue assignment schemes (Table 3.1) with the compositions of all non-calcification tissues replaced with that of agarose gel. The composition of mixed prostate and calcification tissues are replaced with equivalent by mass mixtures of agarose gel and calcification.

\begin{tabular}{llccc}
\hline \hline CT Image & Tissue assignment & $\mathrm{D}_{90}$ & $\mathrm{~V}_{100}$ & $\mathrm{~V}_{150}$ \\
\hline Uncorrected & PC & 0.550 & 0.615 & 0.415 \\
& PC-20\% & 0.656 & 0.732 & 0.623 \\
& PC-5\% & 0.678 & 0.782 & 0.563 \\
STR & & & & \\
& PC & 0.969 & 0.993 & 0.991 \\
& PC-20\% & 0.983 & 0.975 & 0.977 \\
& PC-5\% & 1.000 & 1.036 & 1.073 \\
Median filter & & & & \\
& PC & 0.979 & 0.991 & 0.989 \\
& PC-20\% & 0.980 & 0.992 & 0.989 \\
& PC-5\% & 1.045 & 1.030 & 1.057 \\
Virtual Sinogram & PC & & & \\
& PC-20\% & 0.979 & 0.991 & 0.989 \\
& PC-5\% & 0.990 & 0.986 & 0.991 \\
& & 1.004 & 1.007 & 1.033 \\
Raw Sinogram & PC & & & \\
& PC-20\% & 0.954 & 0.975 & 0.961 \\
& PC-5\% & 0.983 & 0.974 & 0.976 \\
& & 0.980 & 0.994 & 1.011 \\
\hline \hline
\end{tabular}

Table 3.6: Prostate patient data set characteristics in terms of number of seeds , target volume and dose metrics calculated using the "TG-186" scheme (defined in Table 3.1) for which results are independent of MAR (and results are used as the basis of comparison for dose calculations using different MAR methods and tissue assignment schemes).

\begin{tabular}{cccccc}
\hline \hline & & Target & \multicolumn{3}{c}{ "TG-186" } \\
\cline { 4 - 6 } Patient & Seeds & volume $\left(\mathrm{cm}^{3}\right)$ & $\mathrm{D}_{90}(\mathrm{~Gy})$ & $\mathrm{V}_{100}(\%)$ & $\mathrm{V}_{150}(\%)$ \\
\hline 1 & 74 & 41.4 & 123.6 & 84.9 & 68.6 \\
2 & 87 & 55.4 & 167.7 & 93.3 & 76.0 \\
3 & 70 & 34.9 & 145.1 & 90.0 & 67.5 \\
4 & 60 & 28.4 & 163.0 & 93.5 & 75.4 \\
5 & 52 & 30.8 & 149.9 & 91.4 & 64.2 \\
6 & 75 & 41.0 & 198.8 & 98.4 & 83.5 \\
7 & 45 & 24.0 & 167.6 & 96.4 & 62.7 \\
8 & 74 & 27.5 & 244.6 & 99.7 & 93.9 \\
\hline \hline
\end{tabular}


Table 3.7: Summary of patient dose metrics normalized to the "TG-186" metrics (Table 3.6): mean, standard deviation ("S.D."), minimum, and maximum of $\mathrm{D}_{90}, \mathrm{~V}_{100}$ and $\mathrm{V}_{150}$ for the eight patients. Dose distributions are calculated in patient models derived from uncorrected and MAR CT images using one of five tissue assignment schemes of varying complexity (Table 3.1).

\begin{tabular}{|c|c|c|c|c|c|c|c|c|c|c|c|c|c|}
\hline \multirow[b]{2}{*}{ CT Image } & \multirow{2}{*}{$\begin{array}{l}\text { Tissue } \\
\text { assignment }\end{array}$} & \multicolumn{4}{|c|}{$\mathrm{D}_{90}$} & \multicolumn{4}{|c|}{$\mathrm{V}_{100}$} & \multicolumn{4}{|c|}{$\mathrm{V}_{150}$} \\
\hline & & Mean & S.D. & Min & Max & Mean & S.D. & Min & $\operatorname{Max}$ & Mean & S.D. & Min & Max \\
\hline \multirow[t]{5}{*}{ Uncorrected } & Simple-N & 0.8942 & 0.0346 & 0.8312 & 0.9341 & 0.9645 & 0.0209 & 0.9326 & 0.9938 & 0.8857 & 0.0580 & 0.8011 & 0.9776 \\
\hline & Simple-CT & 0.8612 & 0.0333 & 0.8044 & 0.9017 & 0.9501 & 0.0261 & 0.9183 & 0.9900 & 0.8313 & 0.0782 & 0.7359 & 0.9639 \\
\hline & $\mathrm{PC}$ & 0.4898 & 0.0283 & 0.4578 & 0.5398 & 0.5000 & 0.1681 & 0.2608 & 0.8372 & 0.2671 & 0.1181 & 0.1540 & 0.5244 \\
\hline & PC- $20 \%$ & 0.6031 & 0.0290 & 0.5695 & 0.6414 & 0.7048 & 0.1288 & 0.4722 & 0.9184 & 0.4127 & 0.1663 & 0.2362 & 0.7601 \\
\hline & $\mathrm{PC}-5 \%$ & 0.6150 & 0.0284 & 0.5757 & 0.6471 & 0.7405 & 0.1045 & 0.5594 & 0.9250 & 0.4627 & 0.1618 & 0.2760 & 0.7907 \\
\hline \multirow[t]{5}{*}{ STR } & Simple-N & 0.9947 & 0.0025 & 0.9894 & 0.9973 & 0.9986 & 0.0003 & 0.9982 & 0.9990 & 0.9967 & 0.0012 & 0.9948 & 0.9985 \\
\hline & Simple-CT & 0.9843 & 0.0018 & 0.9829 & 0.9875 & 0.9955 & 0.0017 & 0.9930 & 0.9980 & 0.9869 & 0.0051 & 0.9807 & 0.9943 \\
\hline & $\mathrm{PC}$ & 0.9776 & 0.0108 & 0.9519 & 0.9854 & 0.9944 & 0.0027 & 0.9894 & 0.9980 & 0.9786 & 0.0139 & 0.9522 & 0.9945 \\
\hline & PC-20\% & 0.9607 & 0.0039 & 0.9550 & 0.9651 & 0.9889 & 0.0053 & 0.9811 & 0.9960 & 0.9592 & 0.0167 & 0.9334 & 0.9852 \\
\hline & $\mathrm{PC}-5 \%$ & 0.9478 & 0.0103 & 0.9329 & 0.9654 & 0.9850 & 0.0066 & 0.9753 & 0.9938 & 0.9666 & 0.0205 & 0.9344 & 0.9863 \\
\hline \multirow[t]{5}{*}{ Median filter } & Simple-N & 0.9944 & 0.0025 & 0.9894 & 0.9970 & 0.9985 & 0.0003 & 0.9980 & 0.9990 & 0.9965 & 0.0013 & 0.9943 & 0.9982 \\
\hline & Simple-CT & 0.9815 & 0.0022 & 0.9789 & 0.9852 & 0.9947 & 0.0021 & 0.9920 & 0.9977 & 0.9841 & 0.0057 & 0.9767 & 0.9928 \\
\hline & $\mathrm{PC}$ & 0.9749 & 0.0105 & 0.9500 & 0.9832 & 0.9936 & 0.0029 & 0.9884 & 0.9977 & 0.9758 & 0.0140 & 0.9496 & 0.9933 \\
\hline & PC- $20 \%$ & 0.9784 & 0.0029 & 0.9717 & 0.9818 & 0.9940 & 0.0250 & 0.9904 & 0.9977 & 0.9799 & 0.0091 & 0.9647 & 0.9931 \\
\hline & $\mathrm{PC}-5 \%$ & 0.9446 & 0.0153 & 0.9269 & 0.9702 & 0.9830 & 0.0077 & 0.9714 & 0.9933 & 0.9670 & 0.0231 & 0.9304 & 0.9862 \\
\hline \multirow[t]{5}{*}{ Virtual Sinogram } & Simple-N & 0.9939 & 0.0026 & 0.9887 & 0.9967 & 0.9984 & 0.0003 & 0.9977 & 0.9988 & 0.9961 & 0.0013 & 0.9938 & 0.9979 \\
\hline & Simple-CT & 0.9796 & 0.0027 & 0.9761 & 0.9843 & 0.9942 & 0.0023 & 0.9913 & 0.9976 & 0.9821 & 0.0062 & 0.9745 & 0.9919 \\
\hline & $\mathrm{PC}$ & 0.9713 & 0.0159 & 0.9328 & 0.9825 & 0.9931 & 0.0027 & 0.9888 & 0.9972 & 0.9739 & 0.0160 & 0.9386 & 0.9923 \\
\hline & $\mathrm{PC}-20 \%$ & 0.9150 & 0.0097 & 0.9017 & 0.9311 & 0.9734 & 0.0146 & 0.9462 & 0.9913 & 0.9015 & 0.0435 & 0.8221 & 0.9660 \\
\hline & $\mathrm{PC}-5 \%$ & 0.9158 & 0.0134 & 0.8980 & 0.9385 & 0.9738 & 0.0111 & 0.9589 & 0.9896 & 0.9258 & 0.0283 & 0.8888 & 0.9719 \\
\hline \multirow[t]{5}{*}{ Raw Sinogram } & Simple-N & 0.9912 & 0.0043 & 0.9835 & 0.9968 & 0.9977 & 0.0007 & 0.9964 & 0.9987 & 0.9940 & 0.0024 & 0.9903 & 0.9971 \\
\hline & Simple-CT & 0.9792 & 0.0030 & 0.9761 & 0.9848 & 0.9941 & 0.0021 & 0.9912 & 0.9969 & 0.9826 & 0.0057 & 0.9759 & 0.9920 \\
\hline & $\mathrm{PC}$ & 0.9709 & 0.0090 & 0.9551 & 0.9778 & 0.9923 & 0.0031 & 0.9875 & 0.9959 & 0.9732 & 0.0120 & 0.9587 & 0.9890 \\
\hline & $\mathrm{PC}-20 \%$ & 0.9615 & 0.0158 & 0.9253 & 0.9740 & 0.9885 & 0.0071 & 0.9731 & 0.9960 & 0.9553 & 0.0546 & 0.8221 & 0.9878 \\
\hline & $\mathrm{PC}-5 \%$ & 0.9432 & 0.0165 & 0.9158 & 0.9740 & 0.9823 & 0.0067 & 0.9768 & 0.9944 & 0.9613 & 0.0290 & 0.9180 & 0.9878 \\
\hline
\end{tabular}




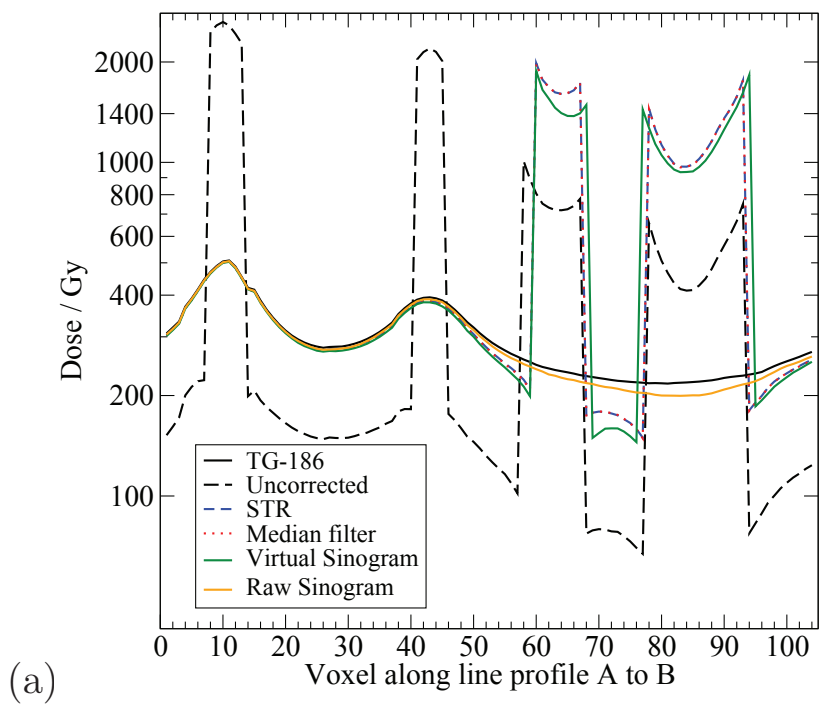

(a)

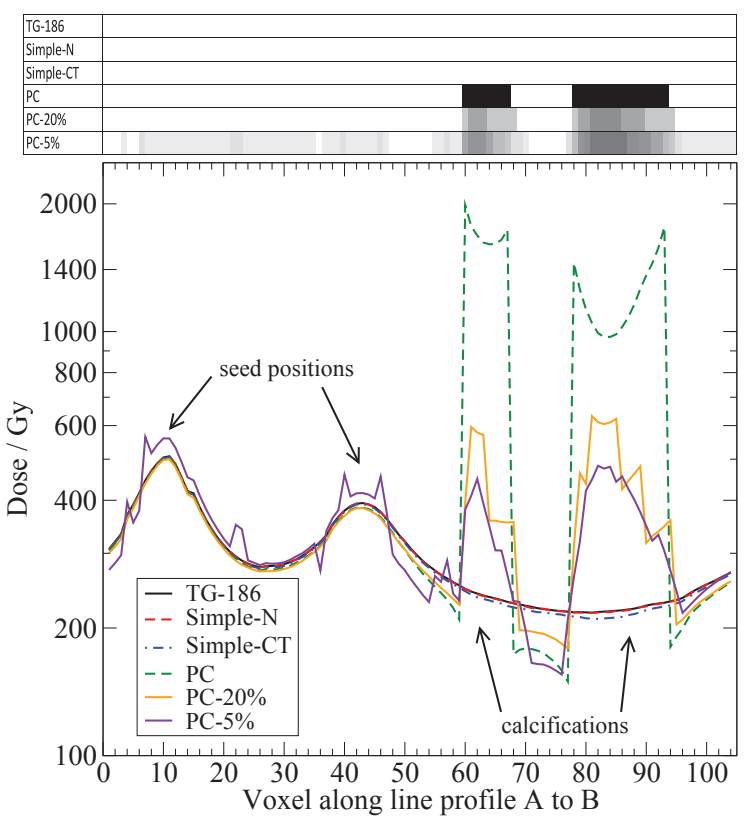

Figure 3.5: Doses calculated to voxels along the line profile A to B, from Figure 3.2(f); error bars representing statistical uncertainty on doses to each voxel are too small to display. (a) "TG-186" basic recommendation and Monte Carlo models derived with the "PC" tissue assignment scheme. (b) "TG-186" and Monte Carlo models derived using the STR CT image and each tissue assignment scheme. Labels indicate regions of the line profile corresponding to seeds and calcifications. Tissues assigned along the line profile in the virtual patient models used for the dose calculations are indicated with white corresponding to prostate, black to calcification and intermediate greyscale to varying fractions of prostate and calcification.

For the same tissue assignment scheme, the choice of MAR method does 
not generally have a large effect (beyond a few percent) on the resulting dose metrics (Table 3.7) and, locally, the differences in post-MAR CT image characteristics (STR: missed dark streaks, median filter: blurring, virtual and raw sinogram: new streaks) have small effect on calculated doses. For example, voxels 0 to 50 in Figure 3.4a show different CT numbers for the different MAR methods but doses calculated differ little, as shown in Figure 3.5a for dose. The exception to this is when tissue assignment schemes differentiate between tissues based on small differences in CT number, e.g., "PC-5\%", and then small differences in CT number lead to different tissue type assigned. Patient models derived using the virtual sinogram with the "PC-20\%" or "PC-5\%" schemes yield relatively lower metrics than other MAR methods since some of the new streaks are mapped to mixed tissues with higher fractions of calcification (Table 3.4). With the detailed "PC-20\%" and "PC-5\%" tissue assignment schemes that require a CT number boundary between prostate and mixed tissue, the value of the CT number threshold used in STR, median filter and virtual sinogram affects the number of voxels assigned to each tissue on the order of a few percent (Section 3.3.2); however, in most cases, the difference in resulting dose metrics is less than $1 \%$.

With tissue assignment schemes which include calcification, high doses are computed to the calcified voxels and there is a shadowing effect with lower doses deposited nearby (e.g., Figure 3.5a voxels 70-78); the magnitude of this effect depends on seed location relative to calcifications. Dose metrics for patient models using calcification schemes ("PC", "PC-20\%" and "PC-5\%") are generally a few percent lower than the no-calcification models. Doses deposited in voxels comprised of mixtures of prostate and calcification ("PC-20\%" and "PC-5\%") are lower than those in pure calcification ("PC") because of lower effective atomic numbers in prostate-calcification mixtures than in pure calcification (e.g., Figure 3.5b voxels 60-100 along the line profile). The very high doses to pure calcifications generally contribute to the tail of the DVH with modest effect on dose metrics. MAR techniques do not always preserve 
calcifications; when a calcification is removed, the dose in the corresponding voxel is underestimated (e.g., Figure 3.5a in the raw sinogram line in voxels $60-100$ along the line profile).

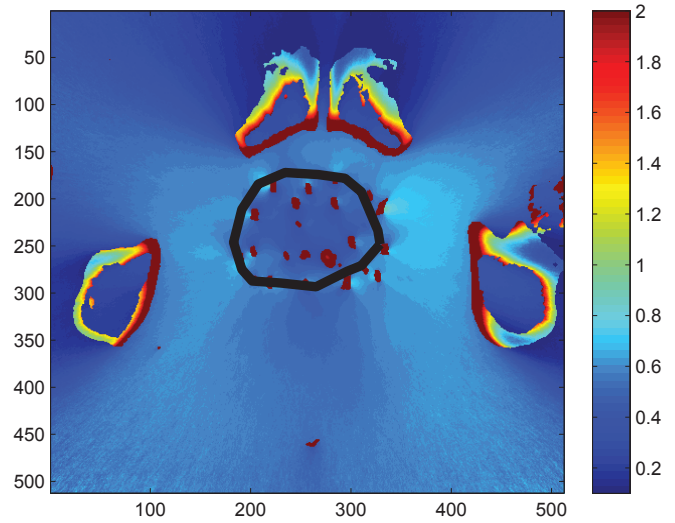

(a)

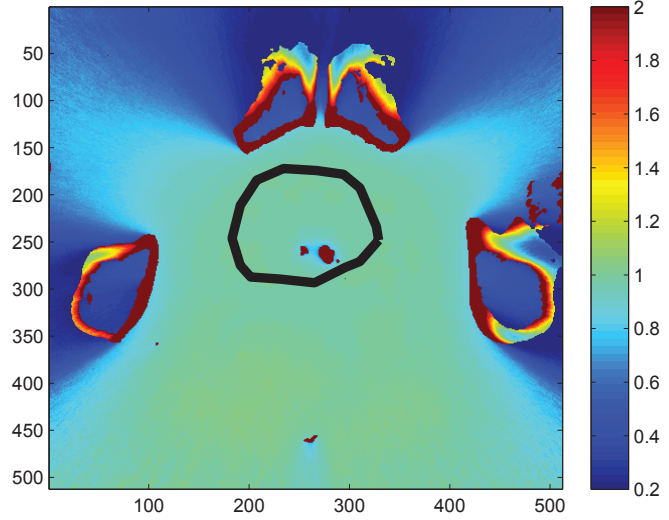

(b)

Figure 3.6: Ratio of doses to voxels in one slice of a prostate patient case from different Monte Carlo virtual patient models, corresponding to the slice shown in Figure 3.2. Axis values correspond to voxel numbers. The CTV contour is overlayed in black. (a) Dose for uncorrected CT image using "PC" over "TG-186" dose. The Uncorrected "PC" model has a much higher dose deposited locally around seeds due to calcification being assigned to seed artifacts in the CT image, resulting in a lower dose to remaining soft tissue. High doses on the edges of the pelvic bones result from the TG-186 scheme not modelling cortical bone outside the target volume. (b) Dose for STR with "PC" over "TG-186" dose. With STR, seed artifacts are removed resulting in a similar dose to "TG-186" for soft tissue in the target. The high dose spots in the CTV correspond to calcifications which are modelled as prostate tissue in the "TG-186" basic recommendation.

\subsection{Discussion}

CT image artifacts are bright spots about seeds and dispersing bright and dark streaks; ultimately, mitigation of the bright spots is most important for accurate dose calculations. Newer CT scanners may include commercial MAR approaches en- 
abling optimization of image acquisition settings to reduce seed artifacts, potentially benefitting prospective dose calculation studies. However, any images suffering from artifacts, e.g., historical CT data needed for retrospective studies (which are necessary to evaluate prescription doses and doses to organs-at-risk for the clinical adoption of $\mathrm{MBDCA}^{26}$ ), require the use of MAR methods such as those employed herein. All four MAR methods eliminate these bright spots in both phantom and prostate patient CT images. Although each MAR method has the potential to preserve calcifications, no technique preserves calcifications universally for the patient datasets considered with some calcifications eliminated near seed positions. When calcifications are included within a tissue assignment scheme, dose metrics are generally lower by a few percent compared to models with no calcifications. The challenge of differentiating prostatic calcifications from image artifacts in CT images, compounded with the dosimetric importance of modelling calcifications, suggests that physician drawn calcification contours should be considered for inclusion in the clinical dose calculation process.

STR is the simplest MAR approach considered; however, it is unable to eliminate dark artifacts near seeds or those streaking outwards. This limitation was not found to be important for the prostate context because there was no differentiation of tissues at low CT number, however it should be considered for e.g., lung or breast. ${ }^{47,48,80,81}$

The median filter eliminates dark streaking artifacts in the regions where it is applied near seeds but not those which are far from seeds (either in the remainder of the target volume or beyond). One could apply the median filter to a more general set of voxels, e.g., the whole treatment volume (as done in Ref. ${ }^{38}$ ), but this eliminates high CT numbers representing calcifications. The median filter smooths CT numbers, which was shown by slightly lower standard deviations of CT numbers in image ROIs (Table 3.3), and resulted in a higher fraction of voxels mapped towards the mean 
prostate CT number; however, there was little effect on dose metrics compared with other MAR methods. This smoothing effect could challenge preservation of soft tissue heterogeneities important for other treatment sites such as breast ${ }^{47,48}$ or lung. ${ }^{29,30}$ The 3D median filter is one of several spatial-domain filters which could be applied to mitigate artifacts from CT images. We have explored other similar methods, such as Gaussian filters, 2D and 3D filters, varied kernel and clip sizes, and have consistently observed comparative blurring effects. The 3D median filter is presented herein based on its superior capability to mitigate the small bright seed spot artifacts. ${ }^{38}$

Seed centroid information is used to guide the STR and median filter MAR methods to maintain integrity of the majority of the CT image voxels not affected by artifacts. One might imagine that the inclusion of seed rotational information could further constrain the MAR methods by reducing the size of the applied region in the radial axis of the seeds. However, the applied MAR region around seeds must be considerably larger than actual seed dimensions to account for the relatively larger size of the observed bright spot artifacts and any small errors in seed centroid position. Seed rotational information cannot be reliably extracted from historical CT images, and has been shown to not significantly affect Monte Carlo dose calculations in the prostate for ${ }^{125}$ I permanent implant brachytherapy. ${ }^{24}$

The virtual sinogram and raw sinogram MAR methods are able to mitigate bright and dark artifacts from the entire CT image, however, they each introduce new broad streaks which vary in prevalence between patients and are most pronounced from the edges of the bones in the prostate patient images. The virtual sinogram MAR resulted in more voxels assigned to higher fractions of calcification in mixed tissues using the "PC-5\%" and "PC-20\%" tissue assignment schemes compared to the other MAR methods, resulting in lower $\mathrm{D}_{90}$ values. The raw sinogram method requires original scanner data which is not often stored by institutions and may be 
in proprietary formats or restricted from the user; it does not appear to offer any advantage over the image-based MAR methods, supporting the use of these simpler techniques. The raw sinogram approach is highly dependent on CT scanner geometry factors, such as the relative positioning of the CT source and image isocentre, and specific geometry values are not generally made available by the vendor. On the other hand, the virtual sinogram approach is less sensitive to CT scanner geometry parameters, and can be adjusted by tens of percent before reconstructed image quality diminishes.

With more complex tissue assignment schemes, which consider calcifications or hybrid prostate and calcified tissues, the application of MAR is critical to prevent patient models in which seeds are encapsulated in calcifications or bone. The type of MAR applied affects the distribution of tissues assigned depending on the type of residual artifacts from the MAR and the level of CT number differentiation used in the tissue assignment scheme. For detailed tissue assignment schemes (e.g., "PC-5\%"), small variations in CT number for different MAR methods have a larger effect than with simpler tissue assignment schemes. The interdependence of MAR technique and tissue assignment scheme suggests that CT number thresholds for MAR and tissue boundaries should be considered together.

For tissue assignment schemes which include calcifications, dose metrics may be wrong by $50 \%$ if these schemes are applied to uncorrected CT images. Dose metrics for MAR-treated images applied a tissue assignment scheme which includes calcifications are generally a few percent lower than for models that do not include calcifications (e.g., "TG-186") which may be due to actual calcifications or from residual artifacts or new streaks (e.g., virtual sinogram) since this was also observed with the calcification-free homogeneous phantom. Previous studies have demonstrated the potential effect of calcifications to be much higher, ${ }^{47,54,78}$ however, only a small number 
of voxels in the target (at most 1\%) were assigned to pure calcification for the patients considered herein.

In this work, doses computed using virtual patient models derived using the "TG-186" scheme (independent of MAR) were used as a basis of comparison for calculations employing various MAR methods and other tissue assignment schemes. With the "TG-186" scheme, dose metrics are all higher than those calculated using CT-derived mass densities, with the exception of the "Simple-N" scheme which is very comparable to "TG-186" using MAR images. Some differences will be due to the higher mean density, $1.07 \mathrm{~g} / \mathrm{cm}^{3}$, for voxels in the prostate of the patient datasets over the nominal density of $1.04 \mathrm{~g} / \mathrm{cm}^{3}$. Alternate "TG-186" models were explored (results not shown) using $1.07 \mathrm{~g} / \mathrm{cm}^{3}$ density in the prostate and resulted in lower $\mathrm{D}_{90}$ dose metrics by up to $1.5 \%$ compared with the $1.04 \mathrm{~g} / \mathrm{cm}^{3}$ scheme. Observed differences between mass densities for the patient datasets considered herein and the nominal prostate density suggests that reference data are uncertain and require further investigation; these data are generally from aged studies based on a limited number of samples and various assumptions. Differences between observed and literature values, coupled with the limited published data, suggest that using CT-derived mass densities may result in more accurate dose estimates than using nominal values, e.g., the "TG-186" approach.

Given the large potential impact of calcification on computed doses, ${ }^{47,54,78}$ tissue assignment schemes should be able to account for diffuse or micro calcifications, and this was the motivation behind the "PC-20\%" and "PC-5\%" schemes. One challenge with applying these schemes is defining CT number boundaries between tissues. In the current work, CT number boundaries were derived using nominal densities from the published literature (for prostate and calcification); however, these values are uncertain (as noted above). With the "PC-5\%" scheme, more voxels were 
assigned to $95 \%$ prostate and $5 \%$ calcification mixture than to pure prostate tissue since the observed mean prostate CT number fell within the CT number bounds of the mixed tissue. This means that schemes such as "PC-5\%" may be too detailed to be used reliably given the uncertainties in nominal tissue mass densities. Recent work suggests large variation in prostatic calcification mass density ${ }^{82}$ and elemental composition, ${ }^{83}$ which will affect tissue assignment schemes and dose calculations; thus, there is a need for further research characterizing prostatic calcifications.

\subsection{Conclusions}

The TG-186 basic approach avoids artifacts by assigning few tissues with uniform literature nominal mass densities, however, it consistently yields clinical metrics several percent higher than detailed MAR-MC approaches, in addition to substantial local dose differences; with more highly calcified patients, differences would be even larger. For more sophisticated approaches allowing multiple tissues and CT-based mass density, the application of a MAR method in deriving a virtual patient model from a CT image is critical in achieving reasonable dose estimates; the choice of MAR technique does not generally have a major effect on computed doses provided bright spot artifacts about seeds are mitigated, as done by all four MAR methods considered. Each of the four MAR methods has limitations: STR does not mitigate low CT number artifacts, median filter blurs the CT image (limiting the preservation of tissue heterogeneities), and both sinogram approaches introduce new streaks throughout the image. The raw sinogram approach does not appear to offer any advantage over the simpler image-based methods, supporting the use of image-based methods in future studies. Limiting STR and median filter application to regions about seeds (rather than global or target volume application) enables preservation of calcifica- 
tions and other heterogeneities, in addition to mitigating artifacts around seeds with positions outside the target volume. There is an interdependence of MAR method and tissue assignment scheme: more complex tissue assignment models, e.g., with small variations in $\mathrm{CT}$ number mapping to different tissues (mixtures of prostate and calcification), are more sensitive to the MAR method applied. Future work, including the studies described in Chapter 4 and Chapter 5, will investigate the application of MAR methods and tissue assignment schemes, and their interdependence, to other brachytherapy treatment sites, and also compare MC and "TG-43" computed doses for treatment volumes and organs-at-risk. 


\section{Chapter 4}

\section{Monte Carlo Dose Calculations for Breast Brachytherapy}

This Chapter explores Monte Carlo dose calculations in permanent implant breast brachytherapy. Comparisons are made between TG-43 and full tissue dose calculations. A sensitivity analysis is performed to explore how dose calculations are affected by metallic artifact reduction, tissue assignment complexity and tissue elemental composition. 


\subsection{Introduction}

Permanent breast seed implants (PBSI) is a form of accelerated partial breast irradiation (APBI) utilizing permanent implant brachytherapy to treat a common form of breast cancer called Ductal Carcinoma In-Situ (DCIS). Some recent studies have begun to retrospectively explore permanent implant breast brachytherapy, generally reporting that the results based on calculations using the TG-43 formalism overestimate dose to the target $\left(\mathrm{D}_{90} \text { by } 10-40 \%\right)^{48,80,81}$ and underestimate dose to the skin $\left(\mathrm{D}_{10} \text { by up to } 30 \%\right)^{80}$ compared to $\mathrm{MC}$ dose calculations. Further, the differences between MC and TG-43 dose calculations are mostly due to tissue modelling effects rather than interseed effects which only reduce $\mathrm{D}_{90}$ by $2-4 \%$ in ${ }^{103} \mathrm{Pd} \mathrm{PBSI} .{ }^{48,80}$ Unfortunately, MC dose calculations are highly dependent on virtual patient models which are non-trivial to derive as they require several modelling assumptions, described in the following, and until now these have not been systematically explored in the published scientific literature for PBSI.

Patient-specific virtual models are typically derived from post-implant CT scans containing bright spot and streaking artifacts due the high atomic number, high-density brachytherapy seeds. Post-metallic artifact reduction (MAR) CT images differ (especially near seeds) depending on MAR technique, causing an interde-

pendence between MAR and subsequent tissue assignment modelling assumptions. ${ }^{84}$ MAR approaches in existing breast brachytherapy studies are unclear ${ }^{80,85}$ or employ a basic thresholding approach. ${ }^{48}$ This is the first study to explore the application of different MAR techniques and their effect on dose distributions for permanent implant breast brachytherapy.

Post-MAR CT images can be used to derive voxelized patient-specific virtual models by mapping CT number to tissue type and mass density. In the breast, 
soft tissue is mainly comprised of adipose or mammary gland but differentiation of these tissues in standard CT scans is challenging, especially near seeds due to metallic artifacts. A common modelling approach is with an artificial hybrid or mixed adipose and gland tissue, ${ }^{47,48,80,81,85}$ however, the fractional distribution of adipose and gland in breast tissue is known to be highly variable between patients $(\sim 30 \% \text { variations })^{86}$ suggesting that a universal hybrid tissue is not representative of all patients. Previous work has shown that the DVH shape differs between average mixed tissue and separately segmented breast models causing $\mathrm{D}_{90}$ to differ by 10$20 \%{ }^{47,48}$ Some existing studies have opted to separately model adipose and gland using a variety of approaches: age-dependent approximations, ${ }^{48}$ patient-specific mass density thresholds ${ }^{87}$ or fixed mass density thresholds. ${ }^{48}$ However, until now, there has been little consideration of sensitivity on computed doses due to the assumed modelling approach to differentiate between adipose and gland tissues in the virtual patient models.

With the aim of establishing a standard, TG-186 recommended tissue compositions relevant for brachytherapy treatment sites. However, several primary resources for tissue compositions exist in the literature, with multiple definitions for tissues existing in the same documents and differing between resources ${ }^{42,44-46,88}$ (Figure 4.1). Previous work ${ }^{81}$ reported a significant sensitivity to breast brachytherapy dose calculations (upwards of 10\% differences in local doses) depending on the selected tissue composition for adipose and gland used to derive mixed tissues. There are multiple elemental compositions and densities in the literature for skin, ${ }^{42,88}$ however, prior to this work, there have been no published studies exploring realistic tissue composition dosimetric effects for this organ-at-risk in breast brachytherapy treatments.

In this Chapter, post-implant CT images of permanent implant breast brachyther- 


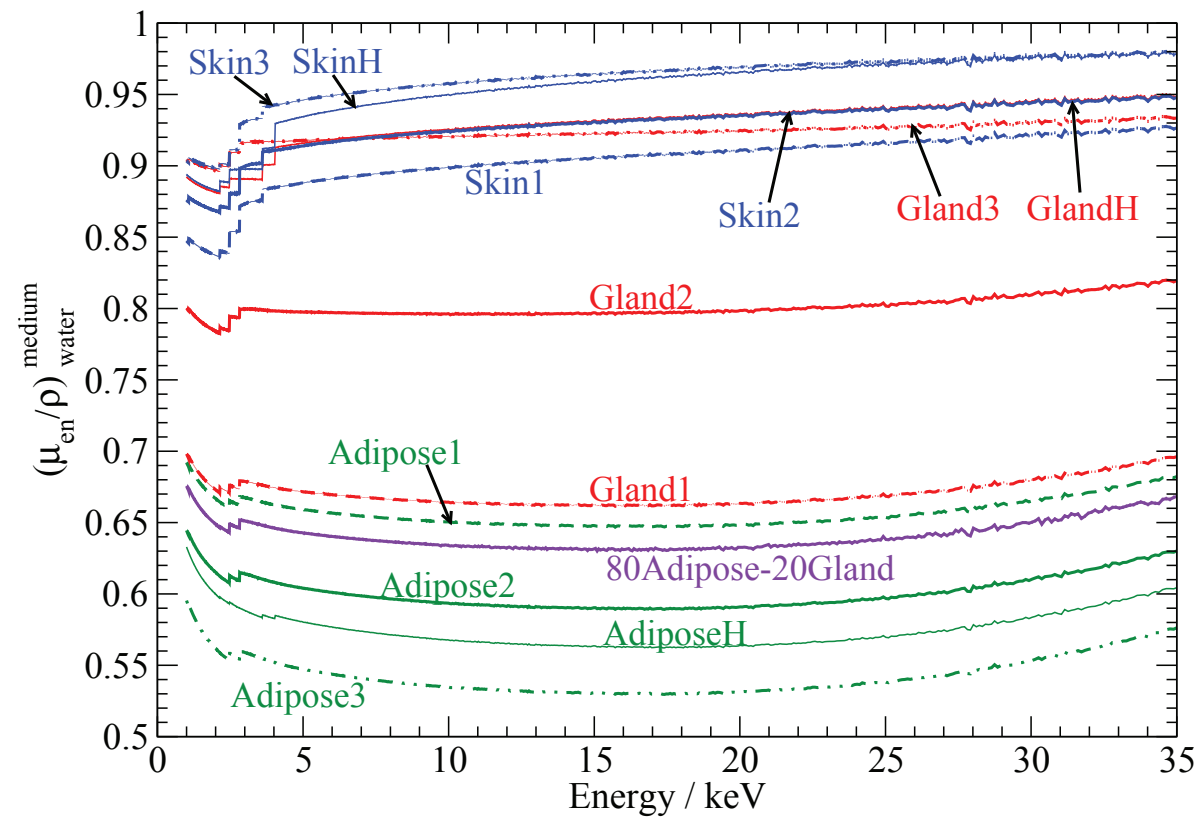

Figure 4.1: Mass energy absorption coefficients for different published compositions of adipose (green), mammary gland (red), skin (blue) and a homogeneous mixed tissue comprised of $80 \%$ adipose and $20 \%$ gland by mass (purple). The compositions labelled " $1,2,3$ " are from ${ }^{42}$ and "2" are the mean compositions recommended by TG186. The compositions labelled " $\mathrm{H}$ " are from ${ }^{88}$ (see Chapter 2 Table 2.2 for the elemental composition of each tissue).

apy patients are used to derive virtual patient-specific models for MC dose calculations. Three different MAR techniques (thresholding, spatial 3D median filter, and virtual sinogram) are applied to the breast for the first time to explore their potential for artifact mitigation in this context while maintaining the ability to differentiate between adipose and gland tissues. Virtual patient models are developed by application of tissue assignment schemes of varying complexity (from mixed tissues to complex segmented models) and employing alternate tissue elemental compositions to uncorrected and post-MAR CT images. We explore the impact of the three MC virtual patient modelling assumptions, namely MAR method, tissue assignment scheme, and tissue composition. Substantial differences between patient-specific MC dose distributions and TG-43 distributions are demonstrated through investigation of local doses, 
DVHs, and dose metrics.

\subsection{Methods}

\subsubsection{Patient Data}

The post-implant CT images are for four PBSI patients treated at The Ottawa Hospital Cancer Centre. Images are centred on the treated breast and contain artifacts due to brachytherapy seeds and fiducial markers on the surface of the breast. Patients were prescribed 90 Gy to the PTV (TG-43 formalism) and treated using 49-77

${ }^{103} \mathrm{Pd}$ Theragenics Model 200 seeds (Chapter 1 Figure 1.1) with air-kerma strengths of 2.50-2.72 U. Patients were pre-screened for treatment with ineligibility criteria including multicentric DCIS sites, target volumes too near the chest wall or skin surface, and indications of microcalcifications in post-lumpectomy imaging. Followup was performed two months after treatment, acquiring CT images of resolution $512 \times 512 \times(42-68)$ voxels corresponding to $(18.9-23.9) \times(18.9-23.9) \times(12.6-18.0) \mathrm{cm}$.

\subsubsection{Metallic artifact reduction}

Prior to deriving virtual patient models from the post-implant CT images, a metallic artifact reduction (MAR) technique is applied to remove the brachytherapy seeds, fiducial markers and the associated bright spot and streaking artifacts from the CT images. Three MAR methods are employed in this work: simple threshold replacement, 3D median filter, and fan beam virtual sinogram (based on the methods presented in Chapter 3).

Simple threshold replacement (STR $)^{29,30,84}$ replaces high CT number voxels 
(above $\left.1.10 \mathrm{~g} / \mathrm{cm}^{3}, 200 \mathrm{HU}\right)$ with a lower value $\left(0.913 \mathrm{~g} / \mathrm{cm}^{3},-72 \mathrm{HU}\right)$ within regions near seed positions (cylinders about each seed centre of radius $5 \mathrm{~mm}$ and extending 5 slices -2 above and 2 below the slice containing the seed). The replacement value is derived from the weighted mean of soft tissue CT numbers in the PTV contours of the four patient images.

The 3D median filter ${ }^{30,38,84}$ mitigates artifacts by replacing the CT number of a voxel if sufficiently different from the local median value with a value from an adjacent slice that is closest to the median. The image is then smoothed by replacing voxel values with local median values. In both steps, if a voxel value is above a threshold of $1.10 \mathrm{~g} / \mathrm{cm}^{3}$ (200 HU), then it is replaced by a lower value of $0.913 \mathrm{~g} / \mathrm{cm}^{3}$ (-72 HU). The median filter is constrained to the seed positions (using the same cylindrical regions as STR), to help preserve genuine steep CT number gradients (like those found at the boundary of soft tissue and bone, lung, or air) and differentiability between adipose and gland tissues. The size of the local regions around each voxel used to compute the local median is within 5 voxels in a CT slice and within 3 slices above or below, and for the second step, within 3 voxels in a CT slice and within 1 slice above or below. The reduction of the local area size in the second step is to minimize blurring of the image.

The fan beam virtual sinogram was derived from earlier studies $28,29,89$ and modified from a parallel trace to a fan beam approach to better model the CT source and detector geometry. ${ }^{84}$ A threshold of $1.94 \mathrm{~g} / \mathrm{cm}^{3}$ (1500 HU) is used to preserve only metal in the reconstructed CT image and then a fan beam transform is applied to generate a metal-only sinogram. The original CT image, containing seed artifacts, is also applied a fan beam transform to produce a virtual sinogram. The metalonly sinogram is subtracted from the virtual sinogram, leaving voids which are filled using cubic spline interpolation, before applying an inverse fan beam transform to 
reconstruct the artifact mitigated CT image. An STR is then applied to further remove any high CT numbers around the seed positions which may have resulted from the cubic spline interpolation.

\subsubsection{Tissue assignment}

Patient-specific virtual models are derived from post-MAR CT images using a tissue assignment scheme which maps the CT number of each image voxel to a tissue type and mass density. Tissue elemental compositions are presented in Chapter 2 Tables 2.1 and 2.2. The contour-guided tissue assignment schemes (Table 4.1) consider three regions: the PTV, the skin and the remaining non-contoured volume. Density to each voxel is assigned from CT number using a measured scanner-specific CT number to mass density calibration curve (Table 4.2) unless otherwise noted.

For consistency with TG-186 recommendations, bones and lungs are mod-

elled with the "skeleton-cortical bone" and "lung -blood-filled" compositions, respectively, from Ref. ${ }^{42}$ The air outside the patient is modelled with $40 \%$ humidity as defined in TG-43U1. ${ }^{20}$ Skin is considered separately from the other soft tissues outside the PTV contour, as advised by TG-186, since it is an organ-at-risk in PBSI and more detailed composition data are available. Skin contours were generated using an in-house code which defines the skin as a band beginning on the outer surface of the patient and extending $5 \mathrm{~mm}$ inward in each CT slice, which is consistent with the 2-5 mm depth assumed in other work. ${ }^{48,80}$ Calcifications are not considered as patients with breast calcifications were ineligible for treatment. Soft tissue is modelled using either a basic homogeneous mixture of $80 \%$ adipose and $20 \%$ mammary gland (by mass), as recommended by TG-186 when differentiation of these tissues on the CT is unreliable, or more realistic segmentation of adipose and gland. 
Table 4.1: Tissue assignment schemes (TAS) used to derive Monte Carlo virtual patient models from CT images. All schemes rely on PTV and skin image contours to guide tissue assignment. One scheme, TG-186 Basic, comes from baseline TG186 recommendations and produces models which are MAR-independent. The other four schemes range in complexity: Simple, Moderate, Detailed1, Detailed2. Tissue compositions are defined in Chapter 2 Tables 2.1 and 2.2. Except for TG-186 Basic, all schemes assign voxel densities from CT number using the calibration in Table 4.2.

\begin{tabular}{|c|c|c|c|c|}
\hline TAS & Contour & Tissue & HU Range & Density \\
\hline \multirow[t]{4}{*}{ TG-186 Basic } & PTV & 80Adipose-20Gland & All & Nominal \\
\hline & Skin & Skin2 & All & Nominal \\
\hline & Remainder & Air & $\leq-880$ & Nominal \\
\hline & & 80Adipose-20Gland & $>-880$ & Nominal \\
\hline \multirow[t]{4}{*}{ Simple } & PTV & 80Adipose-20Gland & All & $\mathrm{CT}$ \\
\hline & Skin & Skin2 & All & $\mathrm{CT}$ \\
\hline & Remainder & Air & $\leq-880$ & $\mathrm{CT}$ \\
\hline & & 80Adipose-20Gland & $>-880$ & $\mathrm{CT}$ \\
\hline \multirow[t]{6}{*}{ Moderate } & PTV & 80Adipose-20Gland & All & $\mathrm{CT}$ \\
\hline & Skin & Skin2 & All & $\mathrm{CT}$ \\
\hline & Remainder & Air & $\leq-880$ & $\mathrm{CT}$ \\
\hline & & Lung & -879 to -600 & $\mathrm{CT}$ \\
\hline & & 80Adipose-20Gland & -599 to 200 & $\mathrm{CT}$ \\
\hline & & Cortical Bone & $>200$ & $\mathrm{CT}$ \\
\hline \multirow[t]{8}{*}{ Detailed1 } & PTV & Adipose2 & $\leq-5$ & $\mathrm{CT}$ \\
\hline & & Gland2 & $>-5$ & $\mathrm{CT}$ \\
\hline & Skin & Skin2 & All & $\mathrm{CT}$ \\
\hline & Remainder & Air & $\leq-880$ & $\mathrm{CT}$ \\
\hline & & Lung & -879 to -600 & $\mathrm{CT}$ \\
\hline & & Adipose2 & -599 to -5 & $\mathrm{CT}$ \\
\hline & & Gland2 & -4 to 200 & $\mathrm{CT}$ \\
\hline & & Cortical Bone & $>200$ & $\mathrm{CT}$ \\
\hline \multirow[t]{8}{*}{ Detailed2 } & PTV & Adipose2 & $\leq-36$ & $\mathrm{CT}$ \\
\hline & & Gland2 & $>-36$ & $\mathrm{CT}$ \\
\hline & Skin & Skin2 & All & $\mathrm{CT}$ \\
\hline & Remainder & Air & $\leq-880$ & $\mathrm{CT}$ \\
\hline & & Lung & -879 to -600 & $\mathrm{CT}$ \\
\hline & & Adipose2 & -599 to -36 & $\mathrm{CT}$ \\
\hline & & Gland2 & -35 to 200 & $\mathrm{CT}$ \\
\hline & & Cortical Bone & $>200$ & $\mathrm{CT}$ \\
\hline
\end{tabular}


Table 4.2: CT number to mass density calibration (rounded to 3 significant figures).

\begin{tabular}{cc}
\hline \hline CT number $(\mathrm{HU})$ & mass density $\left(\mathrm{g} / \mathrm{cm}^{3}\right)$ \\
\hline-1024 & 0.00120 \\
-849 & 0.00120 \\
-706 & 0.155 \\
-535 & 0.296 \\
-99 & 0.884 \\
10 & 1.00 \\
208 & 1.11 \\
467 & 1.33 \\
1234 & 1.81 \\
3520 & 2.88 \\
5750 & 3.94 \\
8000 & 4.98 \\
\hline \hline
\end{tabular}

The mass density threshold for differentiation between adipose and gland tissue is uncertain; different boundary values will result in varying fractional assignments of adipose and gland in the virtual patient models. Average mass densities for adipose and gland tissue are recommended by TG-186 ("Adipose2" $\rho=0.95 \mathrm{~g} / \mathrm{cm}^{3}$ and "Gland2" $\rho=1.02 \mathrm{~g} / \mathrm{cm}^{3}$ ). Our "Detailed1" tissue assignment scheme uses the half way point between these nominal tissue densities $\left(0.985 \mathrm{~g} / \mathrm{cm}^{3},-5 \mathrm{HU}\right)$ to differentiate between adipose and gland. In recent work, an exploration of the healthy breast of ten breast cancer patients ${ }^{48}$ reported a mean adipose density of $0.919 \pm 0.001 \mathrm{~g} / \mathrm{cm}^{3}$ and a mean gland density of $0.992 \pm 0.01 \mathrm{~g} / \mathrm{cm}^{3}$. Our "Detailed2" scheme uses the midpoint of these recently-reported densities $\left(0.956 \mathrm{~g} / \mathrm{cm}^{3}\right)$ to motivate a boundary of $-36 \mathrm{HU}$ to differentiate between adipose and gland. A similar adipose and gland boundary has been used in other studies; a minimum of $0.94 \mathrm{~g} / \mathrm{cm}^{3}$ but varying with patient in Ref. ${ }^{87}$ and $0.949 \mathrm{~g} / \mathrm{cm}^{3}$ in Ref. ${ }^{48}$

The mean adipose and gland tissue elemental compositions recommended by TG-186 ("Adipose2" and "Gland2" in Chapter 2 Table 2.2) have been adopted by several brachytherapy studies ${ }^{47,85,87,90,91}$ - however, compositions for these tissues 
(and others) can differ considerably between literature sources with mass energy absorption coefficients differing by up to tens of percent at ${ }^{103} \mathrm{Pd}$ energies (Figure 4.1). To address this variability of literature tissue compositions we consider four compositions for each of adipose, gland and skin. For each tissue, three compositions are provided by Ref. ${ }^{42}$ (with the middle value recommended by TG-186 and used in our "Detailed1" and "Detailed2" tissue assignment schemes) and one is provided by Ref. ${ }^{88}$ We have defined three alternate versions of the "Detailed2" tissue assignment scheme which use the same mass density boundary to differentiate between adipose and gland, but assume alternate adipose, gland and skin compositions. "Alt1" assumes the Adipose1, Gland3 and Skin1 compositions (highest $\mu_{e n} / \rho$ for adipose and gland, lowest $\mu_{e n} / \rho$ for skin), "Alt2" uses the opposite, Adipose3, Gland1 and Skin3 (lowest $\mu_{e n} / \rho$ for adipose and gland, highest $\mu_{e n} / \rho$ for skin) and "AltH" uses all compositions from: ${ }^{88}$ AdiposeH, GlandH and SkinH.

Reported TG-43 results, "TG-43sim", are MC simulations of TG-43 conditions which model the patient as uniform density water $\left(\rho=0.998 \mathrm{~g} / \mathrm{cm}^{3}\right)$ and omit interseed effects. Alternate calculations are also performed, denoted "Water", which mimic the TG-43 water conditions but include interseed effects. 


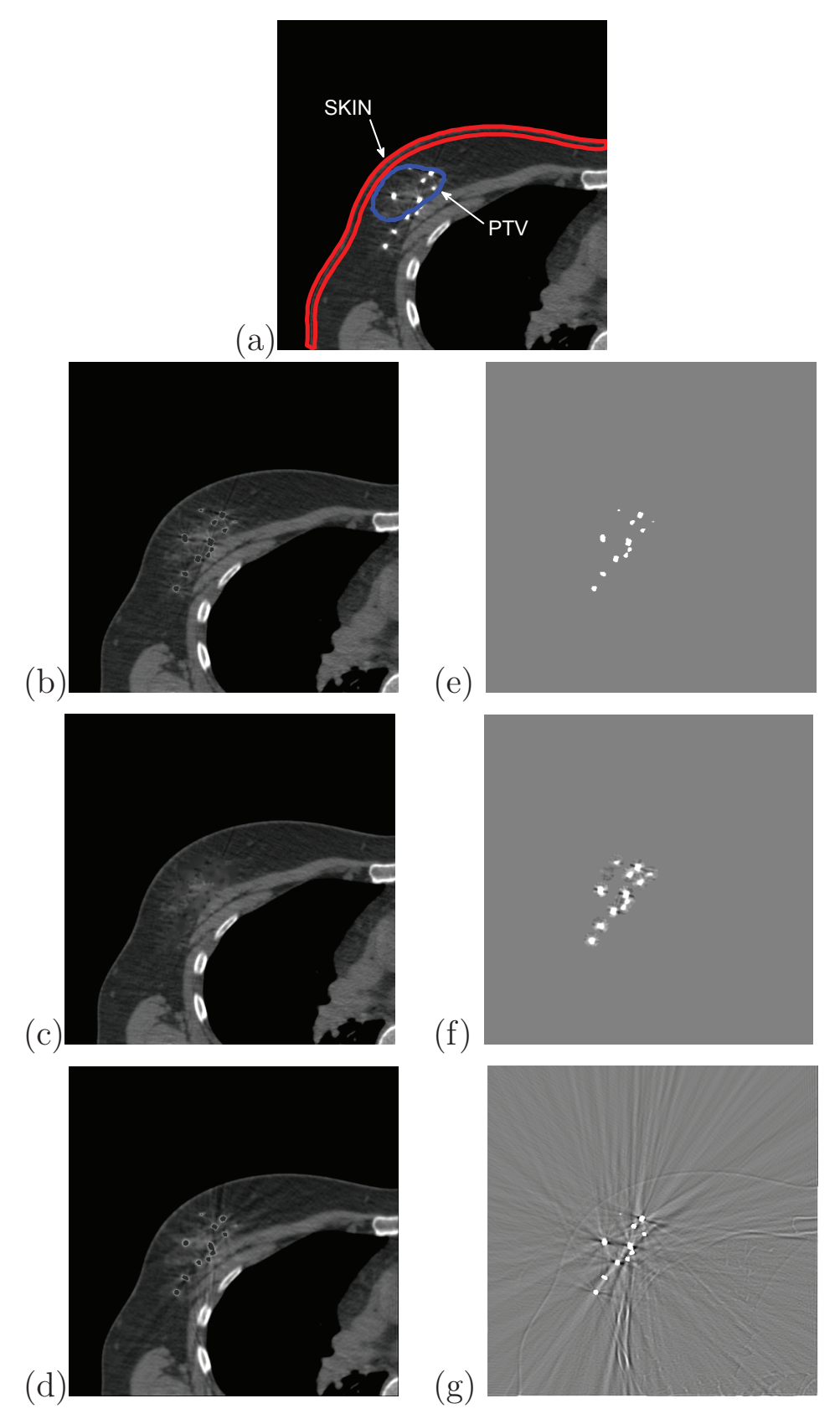

Figure 4.2: (a) Slice through one uncorrected breast patient CT image, showing seed artifacts and contours (PTV, skin) for tissue assignment and dose metrics. The left column is the same breast patient CT image after MAR application: (b) STR, (c) 3D Median Filter, (d) virtual sinogram. The right column gives the difference between the uncorrected and MAR CT image for (e) STR, (f) 3D Median filter and (g) virtual sinogram. Images (a,b,c,d) use a window level of $150 \mathrm{HU}$ and width of $700 \mathrm{HU}$ to emphasise original and residual artifacts. Images (e,f,g) use a window level of $0 \mathrm{HU}$ and width of $400 \mathrm{HU}$ to emphasise any residual or new artifacts. 


\subsubsection{Dose calculations}

The EGSnrc ${ }^{60,92}$ user-code BrachyDose $\mathrm{e}^{57,58}$ is employed to calculate dose distributions with dose to the local voxel medium scored $\left(\mathrm{D}_{m, m}\right)$. Each $\mathrm{MC}$ dose calculation uses $10^{10}$ histories resulting in an uncertainty to the PTV of less than $0.5 \%$ (which is the quadrature sum of uncertainties on voxels within the PTV receiving at least $25 \%$ of the prescription dose).

Dose metrics reported are those considered by the Ottawa Hospital Cancer Centre: $\mathrm{D}_{90}$ is the isodose surrounding $90 \%$ of the PTV, $\mathrm{V}_{100}\left(\mathrm{~V}_{150}, \mathrm{~V}_{200}\right)$ is the percentage of the PTV receiving $100 \%(150 \%, 200 \%)$ of the 90 Gy prescription dose, Dose Homogeniety Index (DHI) is given by $\mathrm{DHI}=1-\left(\mathrm{V}_{150} / \mathrm{V}_{100}\right)$, and $\mathrm{D}_{1 \mathrm{~cm}^{3}}$ is the isodose surrounding the hottest $1 \mathrm{~cm}^{3}$ of skin. An example of the PTV and skin contours used for tissue assignment and to define DVH regions can be seen overlaid onto the CT image in Figure 4.2.

\subsection{Results}

\subsubsection{Metallic artifact reduction}

The uncorrected CT images contain bright spot artifacts around the seed positions, occasional dark regions near the seeds, and less pronounced bright and dark streaks extending outwards. Figure 4.2a is a slice through one uncorrected patient CT image illustrating the metallic artifacts. The bright spots have a typical diameter of 6-10 voxels corresponding to about $2 \mathrm{~mm}$ (seeds are $0.826 \mathrm{~mm}$ in diameter). Figures $4.2 \mathrm{~b}-$ g show the same CT slice after application of the STR, median filter and virtual sinogram MAR techniques: although all MAR methods mitigate the bright spot 
artifacts around seeds, each method leaves characteristic missed or new artifacts. For STR (Fig 4.2b,e), there are residual bright ring artifacts within the area of application which arise from CT numbers below the threshold but higher than the background soft tissue. Further, STR is unable to mitigate low CT numbers which appear as dark spots near the seeds. The median filter (Fig 4.2c,f) can mitigate the dark spots and streaks near the seeds but blurs the CT numbers in the region to which it is applied. The virtual sinogram (Fig 4.2d,g) adds new streaks throughout the image and leaves residual bright ring artifacts around the seeds.

The different MAR methods can yield differences in CT number to individual voxels in excess of $100 \mathrm{HU}$, corresponding to roughly $0.1 \mathrm{~g} / \mathrm{cm}^{3}$, which may be significant for differentiation between adipose and gland. An example of differences in CT number arising with different MAR techniques is provided in Figure 4.3 which gives the CT number line profile through the skin (voxels 5-13 along profile) and through the treated region of the breast. In this figure, many voxels (25-42 and beyond 57 along the profile) are within the regions of application for STR and the median filter. Although STR can potentially affect all voxels near seeds, here the STR line is coincident with the uncorrected line since no voxels are above the 200 HU STR threshold. The median filter is also constrained to regions near the seeds, so its line is coincident with those for STR and uncorrected outside the seed regions; near seeds, the median filter line is different and smoother than the other MAR lines. The virtual sinogram can potentially affect all image voxels and here its line is seen to slightly differ from the uncorrected, STR and median lines in the regions near the seeds and beyond. 


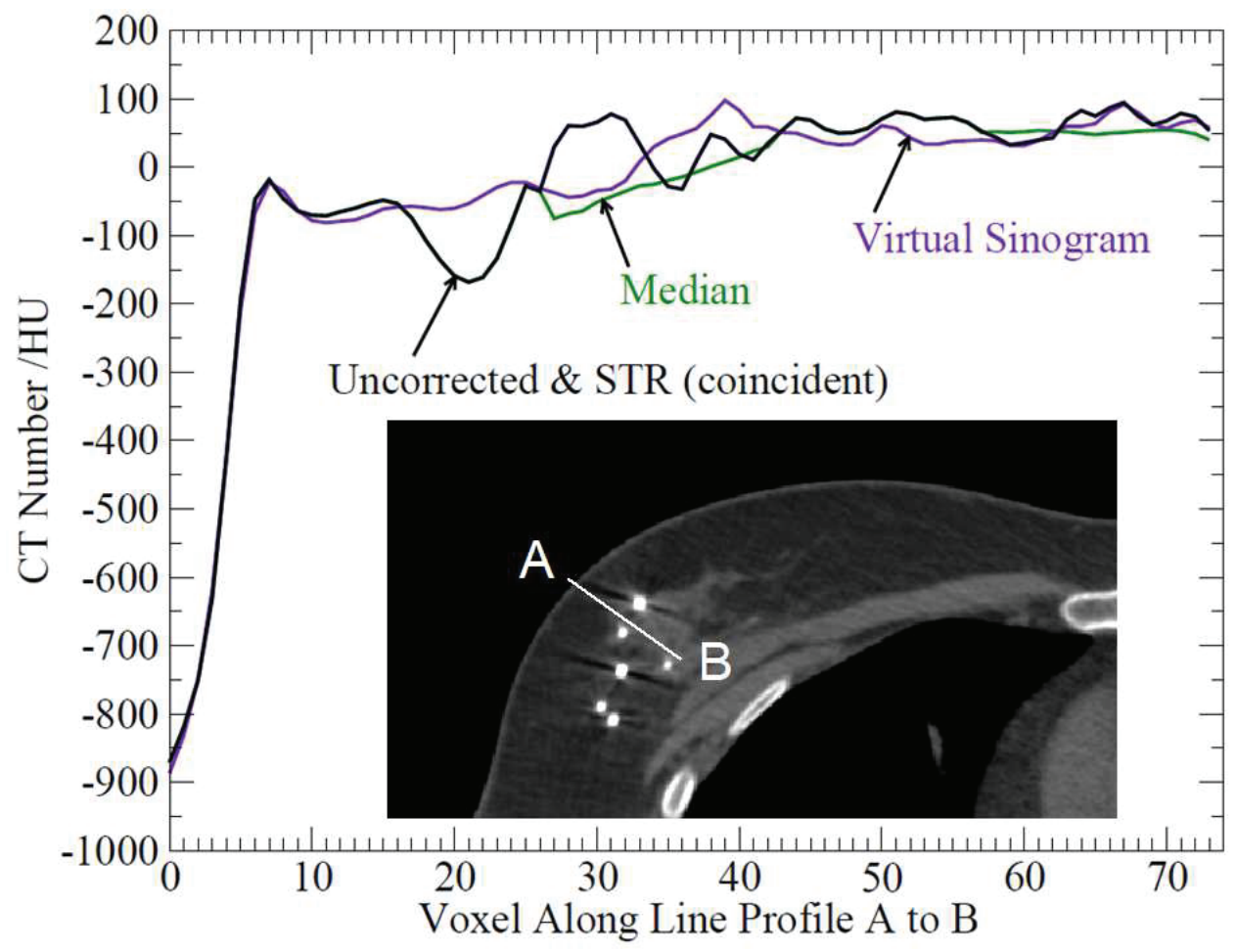

Figure 4.3: For one example patient, CT numbers along line profile A to B, displayed in overlaid CT slice, for uncorrected and MAR-corrected CT images. Line A to B is also used in Figure 4.5,4.6,4.7 and 4.8 dose profiles.

The uncorrected CT images, compared to the MAR images, have a higher mean and larger standard deviation of CT numbers in the treated region of the breast due to the high CT number bright spot artifacts (Figure 4.4). The MAR images have a higher mean and larger standard deviation of CT numbers in image regions near the sources compared to more distal regions of the treated breast, perhaps due to missed or new artifacts as well as real regional differences in breast structure and adipose content. The median MAR images have a lower mean and smaller standard deviation of CT numbers compared to STR and virtual sinogram methods, due to the median filter's blurring effect. The virtual sinogram method is the only MAR technique that may affect regions of the treated breast far from the sources (as STR and median filter are constrained by seed position); CT numbers are similar (with 
small differences due to new streaks) in distal image regions as the uncorrected, STR and median MAR methods.

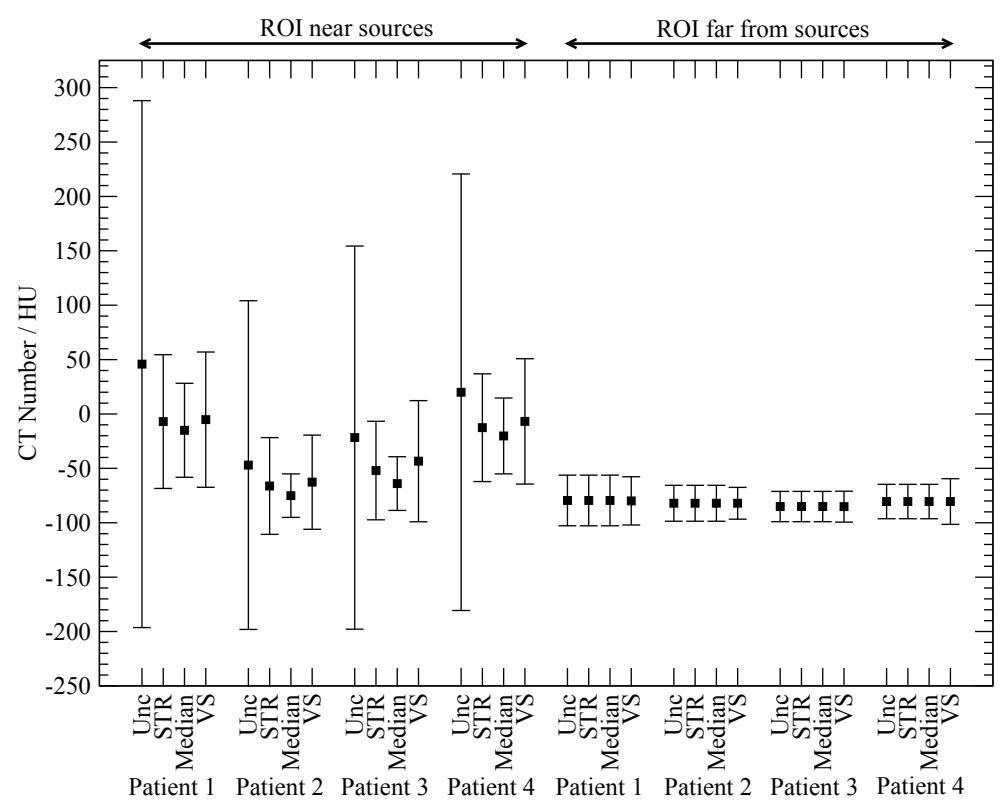

Figure 4.4: Mean data points and standard deviations (error bars) of CT numbers in two example regions of interest (ROI) for each patient CT image after application of different MAR methods. The two ROIs are either near the brachytherapy sources (left) or far from the sources (right) but still in the treated breast. ROIs are defined in one CT slice and range in size from 2144 - 4992 voxels.

\subsubsection{Virtual patient model tissue assignment}

Table 4.3 provides the fraction of voxels within the PTV assigned to adipose or gland for uncorrected and different MAR-corrected CT images using the "Detailed1" and "Detailed2" tissue assignment schemes. Varying the MAR method (with a fixed TAS) changes the fractional assignment of adipose and gland for any given patient by 1 17\%. Using uncorrected CT images generally causes a lower assignment of adipose (by up to 17\%) compared to adipose assigned using MAR-corrected CT images because high CT number seed artifacts (in uncorrected images) are mapped to gland. The 
median MAR virtual patient models have more adipose compared to STR and virtual sinogram since this MAR method comparatively lowers the mean CT number in regions of the image near the sources (Figure 4.4). Alternating between the detailed tissue assignment schemes (with a fixed MAR) can change the fractional adipose and gland assignment for individual patients by 15 - 23\% (about 15-20\% when averaging over all MAR images - bottom rows of Table 4.3). Comparing the relative adipose assignment between patients suggests that the inter-patient variability of adipose content in the PTV may be over $20 \%$.

Table 4.3: The percentage of voxels in virtual patient model PTV volumes assigned to either adipose or gland tissue when models are derived using the "Detailed1" and "Detailed2" tissue assignment schemes with uncorrected or MAR-corrected CT images. Both the "Detailed1" and "Detailed2" schemes separately assign adipose and gland to the virtual patient models but differ by the mass density threshold used for differentiation.

\begin{tabular}{lcccccc}
\hline \hline \multirow{2}{*}{ CT Image } & Tissue & \multicolumn{4}{c}{ \% Adipose/Gland } & Mean \\
\cline { 3 - 6 } & Assignment & P1 & P2 & P3 & P4 & \\
\hline Uncorrected & Detailed1 & $61.4 / 38.6$ & $71.7 / 28.3$ & $75.7 / 24.3$ & $54.3 / 45.7$ & $65.8 / 34.2$ \\
& Detailed2 & $43.1 / 56.9$ & $56.4 / 43.6$ & $60.1 / 39.9$ & $37.4 / 62.6$ & $49.2 / 50.8$ \\
\multirow{3}{*}{ STR } & & & & & \\
& Detailed1 & $64.2 / 35.8$ & $76.2 / 23.8$ & $78.4 / 21.6$ & $56.9 / 43.1$ & $68.9 / 31.1$ \\
\multirow{4}{*}{ Median } & Detailed2 & $45.9 / 54.1$ & $60.9 / 39.1$ & $62.8 / 37.2$ & $39.9 / 60.1$ & $52.4 / 47.6$ \\
& & & & & & \\
\multirow{2}{*}{ VS } & Detailed1 & $69.8 / 30.2$ & $87.7 / 12.3$ & $86.7 / 13.3$ & $60.5 / 39.5$ & $76.2 / 23.8$ \\
& Detailed2 & $47.3 / 52.7$ & $73.1 / 26.9$ & $69.4 / 30.6$ & $41.6 / 58.4$ & $57.9 / 42.1$ \\
& & & & & & \\
Mean(MAR only) & Detailed1 & $63.4 / 36.6$ & $70.8 / 29.2$ & $74.1 / 25.9$ & $56.1 / 43.9$ & $66.1 / 33.9$ \\
& Detailed2 & $44.5 / 55.5$ & $56.4 / 43.6$ & $58.3 / 41.7$ & $38.4 / 61.6$ & $49.4 / 50.6$ \\
& & & & & & \\
\hline \hline
\end{tabular}




\subsubsection{Dose calculations}

\section{Metallic Artifact Reduction}

There can be considerable differences in doses calculated using virtual patient models derived from uncorrected CT images compared to those derived from post-MAR CT images due to the unrealistic assignment of high density tissue to artifact-affected regions encompassing seeds (uncorrected images). Doses to individual voxels are generally much higher near the seeds and several Gray lower elsewhere in uncorrected models compared to post-MAR models when using the same tissue assignment scheme (Figure 4.5a,b). Comparing uncorrected relative to MAR models, the PTV DVH (Figure 4.5d) is underestimated and PTV dose metrics are affected: $\mathrm{D}_{90}$ is lower by up to $5 \%$ and $20 \%, \mathrm{~V}_{100}$ is lower by up to $4 \%$ and $19 \%, \mathrm{~V}_{200}$ is lower by up to $8 \%$ and $29 \%$ and DHI is higher by up to $2 \%$ and $7 \%$ (using "Simple" and "Detailed1" respectively) (Table 4.4). In the skin, comparing uncorrected relative to MAR models underestimates the DVH (Figure 4.5e) and lowers $\mathrm{D}_{1 \mathrm{~cm}^{3}}$ by up to $5 \%$ and $19 \%$ (using "Simple" or "Detailed1") (Table 4.5).

After applying a MAR method to mitigate artifacts, local doses to individual voxels are generally comparable between MAR methods, except when differences in CT number lead to differences in tissue assignment. An example of this can be seen in voxels 26-30 along the line profile in Figure 4.3 where median and virtual sinogram images have CT numbers below the $-35 \mathrm{HU}$ threshold so adipose tissue is assigned whereas STR and uncorrected images have gland assigned. In the corresponding voxels, the doses for median and virtual sinogram models are lower compared to STR and uncorrected models due to the difference in tissue assignment (Figure 4.5a). Variations in the assignment of adipose and gland resulting from minor differences in $\mathrm{CT}$ number in the different post-MAR images yields dose distributions which are 
similar except for isolated voxels which can differ by tens of percent (Figure 4.5c). Despite these local differences, which can be several Gray, the choice of MAR method has little effect on DVHs (Figure 4.5d,e) and moderate effect on dose metrics (Tables 4.4 and 4.5$)$. In the PTV, with a fixed TAS, $\mathrm{D}_{90}, \mathrm{~V}_{100}, \mathrm{~V}_{200}$ and DHI vary between MAR methods by up to $6 \%, 2 \%, 10 \%$ and $9 \%$ respectively. In the skin, with a fixed TAS, the largest difference between the $\mathrm{D}_{1 \mathrm{~cm}}{ }^{3}$ metric is $6 \%$. 

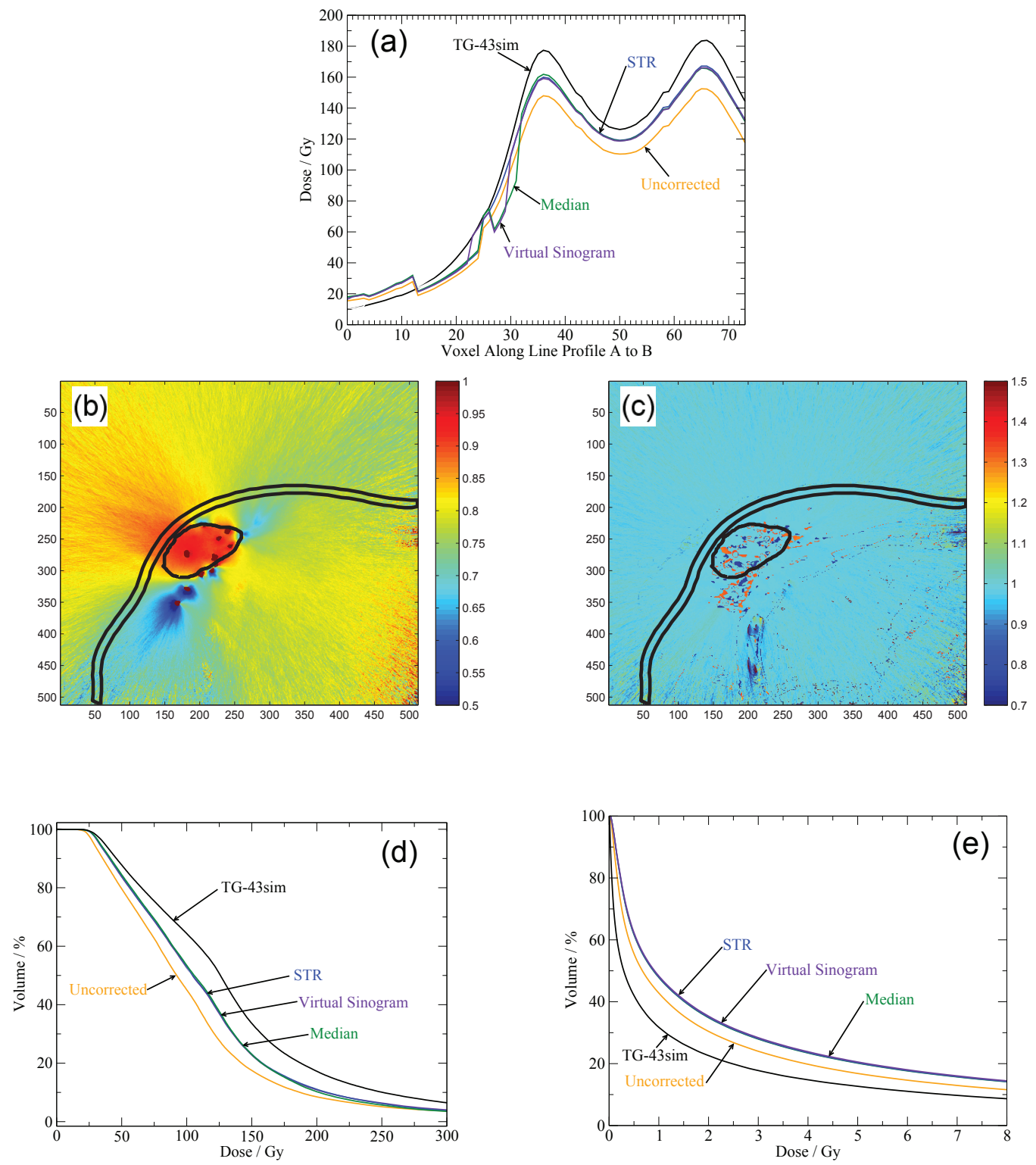

Figure 4.5: Doses calculated using virtual patient models derived using different MAR methods. "TG-43sim" results are included as a basis of comparison. (a) Dose along the line profile shown in Figure 4.3. (b) Dose ratio "Uncorrected+Detailed2" / "STR+Detailed2" showing the effect of deriving virtual patient models from uncorrected or MAR CT images (corresponding to the slice and patient shown in Figure 4.2, with the PTV and skin contours overlaid for reference). (c) Dose ratio "Virtual Sinogram+Detailed2" / "STR+Detailed2" showing the difference between two MAR methods. (d) PTV and (e) skin DVHs for one patient using the "Detailed2" tissue assignment scheme with varying MAR methods. 
Table 4.4: PTV dose metrics $\mathrm{D}_{90}, \mathrm{~V}_{100}, \mathrm{~V}_{200}$ and DHI relative to those for "TG-43sim" for four breast brachytherapy patients (P1-4) computed in virtual patient models derived from different MAR and TAS (TAS described in Table 4.1). Absolute "TG43sim" dose metrics are provided in the first row $\left(\mathrm{D}_{90}, \mathrm{~V}_{100}, \mathrm{~V}_{200}\right.$ and DHI in units of Gy, \%, \% and unitless, respectively). Alternate versions of "Detailed2" scheme, denoted "Alt1", "Alt2" and "AltH" employ alternate elemental compositions for adipose, gland and skin tissues (Chapter 2 Table 2.2).

\begin{tabular}{|c|c|c|c|c|c|c|c|c|c|c|c|c|c|c|c|c|c|}
\hline MAR & TAS & \multicolumn{4}{|c|}{$\mathrm{D}_{90}$} & \multicolumn{4}{|c|}{$V_{100}$} & \multicolumn{4}{|c|}{$V_{200}$} & \multicolumn{4}{|c|}{ DHI } \\
\hline & Water & 0.98 & 0.96 & 0.97 & 0.98 & 0.99 & 0.99 & 0.99 & 0.99 & 0.97 & 0.96 & 0.97 & 0.95 & 1.04 & 1.04 & 1.03 & 1.05 \\
\hline & TG-186 Basic & 0.92 & 0.89 & 1.04 & 0.95 & 0.88 & 0.94 & 0.94 & 0.90 & 0.49 & 0.70 & 0.72 & 0.43 & 1.62 & 1.46 & 1.39 & 1.71 \\
\hline \multirow{3}{*}{ Uncorrected } & Moderate & 0.81 & 0.76 & 0.93 & 0.83 & 0.75 & 0.87 & 0.87 & 0.75 & 0.37 & 0.55 & 0.56 & 0.31 & 1.76 & 1.93 & 1.58 & 2.12 \\
\hline & Detailed1 & 0.76 & 0.70 & 0.84 & 0.76 & 0.70 & 0.84 & 0.83 & 0.73 & 0.45 & 0.52 & 0.53 & 0.42 & 1.53 & 2.01 & 1.68 & 1.79 \\
\hline & Detailed2 & 0.77 & 0.70 & 0.83 & 0.78 & 0.75 & 0.85 & 0.84 & 0.77 & 0.50 & 0.57 & 0.60 & 0.44 & 1.49 & 1.92 & 1.59 & 1.76 \\
\hline \multirow{4}{*}{ STR } & Detailed2 & 0.89 & 0.84 & 0.94 & 0.89 & 0.86 & 0.94 & 0.91 & 0.91 & 0.66 & 0.72 & 0.71 & 0.63 & 1.30 & 1.50 & 1.30 & 1.41 \\
\hline & Alt1 & 0.91 & 0.85 & 0.93 & 0.89 & 0.90 & 0.95 & 0.92 & 0.94 & 0.81 & 0.79 & 0.77 & 0.81 & 1.10 & 1.33 & 1.20 & 1.22 \\
\hline & & 0.86 & 0.82 & 0.95 & 0.87 & 0.81 & 0.91 & 0.89 & 0.87 & 0.49 & 0.63 & 0.62 & 0.44 & 1.57 & 1.71 & 1.45 & 1.71 \\
\hline & AltH & 0.85 & 0.79 & 0.86 & 0.83 & 0.84 & 0.93 & 0.89 & 0.90 & 0.81 & 0.72 & 0.71 & 0.84 & 1.00 & 1.56 & 1.27 & 1.17 \\
\hline \multirow[t]{4}{*}{ Median } & Simple & 0.92 & 0.90 & 1.05 & 0.94 & 0.88 & 0.94 & 0.94 & 0.89 & 0.49 & 0.71 & 0.72 & 0.42 & 1.63 & 1.43 & 1.39 & 1.75 \\
\hline & Mode & 0.92 & 0.90 & 1.05 & 0.9 & 0.88 & 0.94 & 0.94 & 0.8 & 0.49 & 0.71 & 0.71 & 0.4 & 1.63 & 1.43 & 1.40 & 1.76 \\
\hline & Detailed1 & 0.89 & 0.87 & 0.99 & 0.88 & 0.86 & 0.94 & 0.91 & 0.89 & 0.54 & 0.69 & 0.63 & 0.55 & 1.44 & 1.48 & 1.46 & 1.51 \\
\hline & Detailed2 & 0.90 & 0.87 & 0.97 & 0.90 & 0.87 & 0.94 & 0.91 & 0.91 & 0.64 & 0.72 & 0.69 & 0.60 & 1.30 & 1.42 & 1.35 & 1.43 \\
\hline
\end{tabular}


Table 4.5: Skin $\mathrm{D}_{1 \mathrm{~cm}^{3}}$ dose metric relative to that for "TG-43sim" for four breast brachytherapy patients (P1-4) computed in virtual patient models derived from different MAR and TAS (TAS described in Table 4.1). Absolute "TG-43sim" $\mathrm{D}_{1 \mathrm{~cm}^{3}}$ metric (unit Gy) are provided in the first row. Alternate versions of "Detailed2" scheme, denoted "Alt1", "Alt2" and "AltH" employ alternate elemental compositions for adipose, gland and skin tissues (Chapter 2 Table 2.2).

\begin{tabular}{llcccc}
\hline \hline \multirow{3}{*}{ MAR } & & \multicolumn{4}{c}{ Skin $\mathrm{D}_{1 \mathrm{~cm}^{3}}$} \\
\cline { 3 - 6 } & TAS & P1 & P2 & P3 & P4 \\
\hline \multirow{5}{*}{ Uncorrected } & 30.6 & 75.4 & 51.6 & 28.2 \\
& Water & & & & \\
& TG-186 Basic & 1.34 & 0.96 & 0.97 & 0.97 \\
& Simple & 1.21 & 1.34 & 1.38 \\
& Moderate & 1.18 & 1.14 & 1.31 & 1.34 \\
& Detailed1 & 1.16 & 1.00 & 1.12 & 1.16 \\
& Detailed2 & 1.13 & 0.97 & 1.09 & 1.12 \\
STR & & & & & \\
& Simple & 1.35 & 1.20 & 1.35 & 1.38 \\
& Moderate & 1.35 & 1.20 & 1.35 & 1.38 \\
& Detailed1 & 1.35 & 1.15 & 1.34 & 1.32 \\
& Detailed2 & 1.32 & 1.12 & 1.30 & 1.28 \\
& Alt1 & 1.19 & 1.02 & 1.17 & 1.13 \\
& Alt2 & 1.48 & 1.26 & 1.48 & 1.47 \\
Median & AltH & 1.32 & 1.09 & 1.30 & 1.23 \\
& & & & & \\
& Simple & 1.36 & 1.21 & 1.36 & 1.39 \\
& Moderate & 1.36 & 1.21 & 1.36 & 1.39 \\
& Detailed1 & 1.39 & 1.19 & 1.38 & 1.35 \\
VS & Detailed2 & 1.35 & 1.15 & 1.34 & 1.29 \\
& & & & & \\
& Simple & 1.35 & 1.19 & 1.33 & 1.38 \\
& Moderate & 1.35 & 1.20 & 1.33 & 1.38 \\
& Detailed1 & 1.34 & 1.12 & 1.30 & 1.32 \\
& Detailed2 & 1.30 & 1.10 & 1.26 & 1.27 \\
\hline \hline
\end{tabular}




\section{Tissue Assignment Scheme}

For the basic schemes which assign mixed adipose and gland tissue ("TG-186 Basic", "Simple" and "Moderate"), local doses to individual voxels (Figure 4.6a), DVHs (Figure 4.6d,e) and metrics (Tables 4.4 and 4.5) are typically within a few percent of each other (excluding results derived from uncorrected CT images). The "TG-186" scheme (which assigns a uniform literature-derived mass density to each tissue) produces results which are comparable to the "Simple" scheme (which derives voxel mass

density from CT number) even though the nominal density of 80Adipose-20Gland $\left(0.96 \mathrm{~g} / \mathrm{cm}^{3}\right)$ is higher than the average PTV density observed for these patients $\left(0.913 \pm 0.018 \mathrm{~g} / \mathrm{cm}^{3}\right)$. Doses calculated with the "Moderate" scheme deviate from the two simpler schemes when uncorrected CT images are used to derive virtual patient models because the "Moderate" scheme causes seeds outside the PTV region to be modelled as encased in bone (due to bright spot seed artifacts), resulting in considerable attenuation of photons emitted from these sources.

Doses calculated using the two more realistic segmented "Detailed1" and "Detailed2" schemes which separately assign adipose and gland differ considerably from the simpler schemes both locally and in overall DVHs and metrics. The doses calculated to voxels which are assigned the hybrid 80Adipose-20Gland tissue (simpler schemes) are different by about 10-20\% from the voxels in the segmented models which separately assign adipose or gland (Figure 4.6b). 

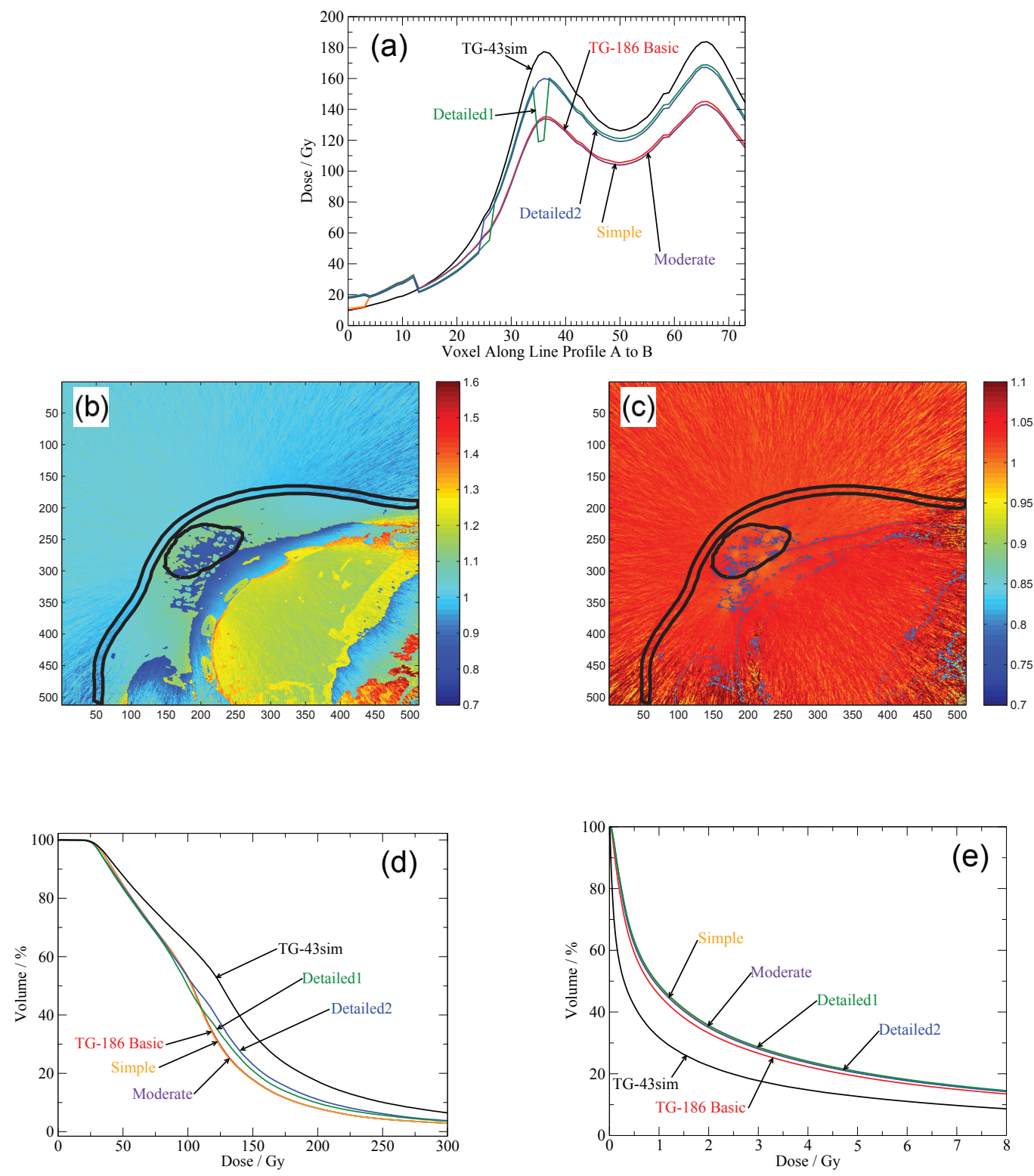

Figure 4.6: Doses calculated for one patient using the "STR" MAR method and various virtual patient models employing different tissue assignment schemes. "TG43sim" results are included as a basis of comparison. (a) Dose along the line profile shown in Figure 4.3. (b) "STR+Moderate" / "STR+Detailed2" showing the effect of modelling 80Adipose-20Gland mixed tissue instead of the separate assignment of adipose and gland. (c) "STR+Detailed1" / "STR+Detailed2" showing differences due to varying the adipose and gland segmentation threshold. (d) PTV and (e) skin DVHs from models using various tissue assignment schemes which differ by the assigned tissue types and density thresholds. 
Between the "Detailed1" and "Detailed2" schemes, which only differ by the mass density threshold used to delineate adipose and gland, large differences of up to tens of Gray in doses to voxels can be seen when virtual patient models differ in assignment of adipose and gland to these voxels (Figure 4.6a,c). Differences can also be seen in the PTV DVHs between these schemes (Figure 4.6d), but mostly in the higher dose regions. In the PTV, using the "Detailed2" scheme relative to "Detailed1" (with a fixed MAR), $\mathrm{D}_{90}, \mathrm{~V}_{100}, \mathrm{~V}_{200}$ and $\mathrm{DHI}$ vary by up to $3 \%, 3 \%, 19 \%$ and $10 \%$, respectively. Skin DVHs are very similar between the "Detailed1" and "Detailed2" schemes (Figure 4.6e). The "Detailed2" scheme, relative to "Detailed1", returns a higher $\mathrm{D}_{1 \mathrm{~cm}} \mathrm{c}^{3}$ metric by up to $4 \%$ (Table 4.5 ).

\section{Tissue Composition}

Dose variations due to different tissue compositions, expected based on behaviour of mass-energy absorption coefficients (Figure 4.1), are observed in virtual patient models which use the three alternate versions of the "Detailed2" scheme, "Alt1", "Alt2" and "AltH" which employ different literature-derived adipose, gland and skin compositions. These alternate schemes result in considerable variations in local doses, DVHs and dose metrics compared to the "Detailed2" scheme. Figure 4.7a illustrates how doses to individual voxels in the PTV and skin can vary by up to $28 \%$ between these alternate schemes. Doses to large segments of contoured regions (PTV and skin) can differ by tens of percent depending on the tissue compositions in the MC models (Figure 4.7b). PTV and skin DVHs (Figures 4.7c,d) differ between these alternate schemes. PTV dose metrics vary between alternate schemes by up to $11 \%$ for $\mathrm{D}_{90}$, up to $10 \%$ for $\mathrm{V}_{100}$, up to $90 \%$ for $\mathrm{V}_{200}$ and up to $57 \%$ for DHI. The skin $\mathrm{D}_{1 \mathrm{~cm}}{ }^{3}$ metric varies by $30 \%$ between alternate schemes (Tables 4.4 and 4.5 ). 

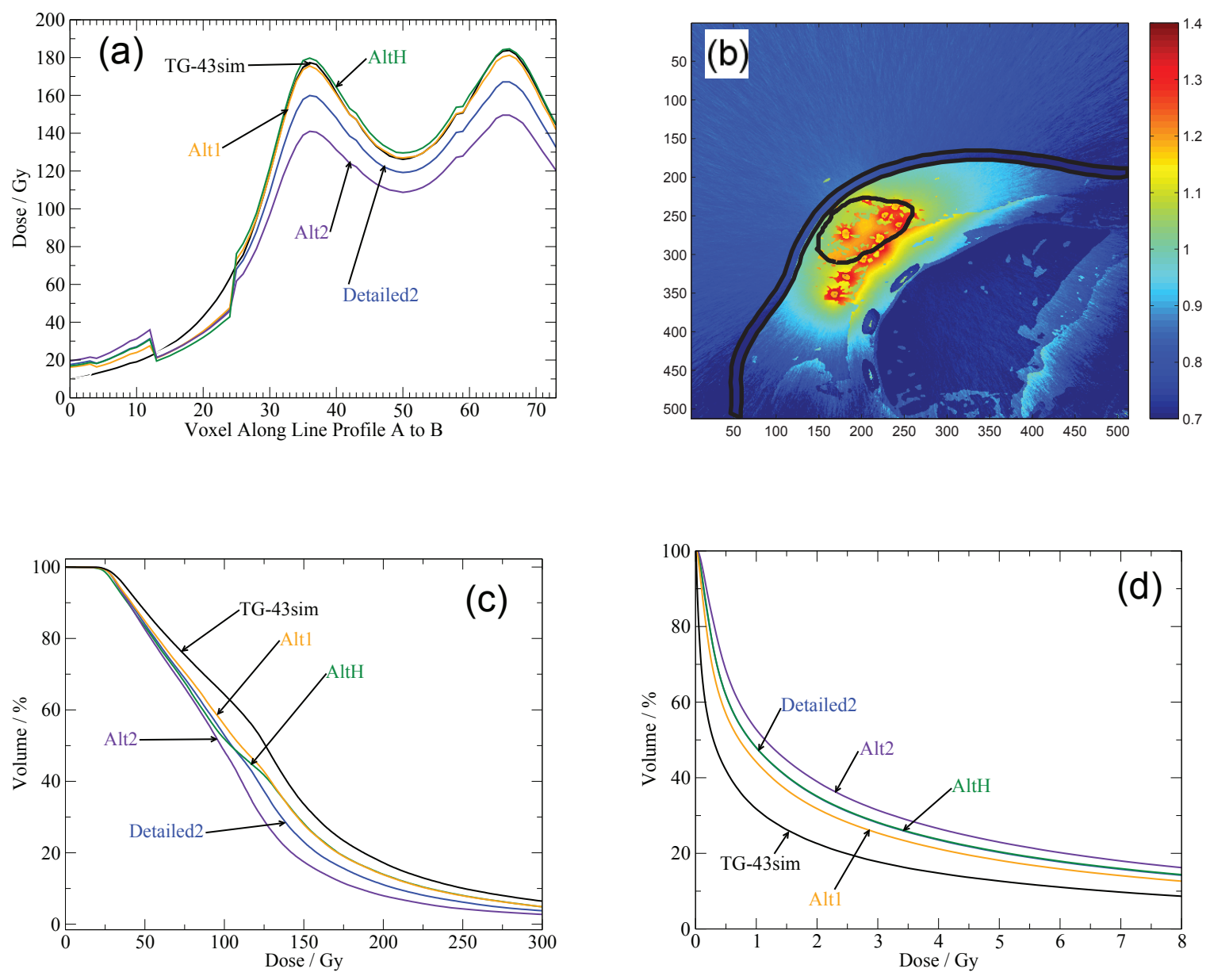

Figure 4.7: Doses calculated for one patient using the "STR" MAR method and various virtual patient models which use alternative adipose, gland and skin compositions. "TG-43sim" results are included as a basis of comparison. (a) Dose along the line profile shown in Figure 4.3. (b) Dose ratio "STR+Alt1" / "STR+Alt2" showing the effect of employing different tissue compositions. (c) PTV and (d) skin DVHs from models using various tissue assignment schemes which employ different tissue compositions. 


\section{TG-43 vs Monte Carlo}

Generally, "TG-43sim" doses to individual voxels in the PTV are higher by up to tens of Gray (tens of percent) compared to the Monte Carlo models which use MAR CT images and assign tissues (Figure 4.8a,b). In the PTV, "TG-43sim" overestimates the DVH (Figure 4.8d) and dose metrics. The "TG-43sim" $\mathrm{D}_{90}$ is higher by up to $12 \%$ and $22 \%, \mathrm{~V}_{100}$ is higher by up to $14 \%$ and $20 \%, \mathrm{~V}_{200}$ is higher by up to $132 \%$ and $87 \%$ and DHI is lower by up to $42 \%$ and $38 \%$ compared to the "TG-186 Basic" and either "Detailed" model respectively (Table 4.4 and Figure 4.9).

In the skin, local "TG-43sim" doses can be lower by several Gray (tens of percent) compared to the basic and detailed Monte Carlo models (Figure 4.8a,b) because the MC models cause a higher photon fluence to reach the skin which compensates for the reduced backscatter contribution and higher mass energy absorption coefficient of water compared to skin tissue. The $\mathrm{D}_{1 \mathrm{~cm}^{3}}$ metric is up to $38 \%$ and $39 \%$ higher calculated with the "TG-186 Basic" and either "Detailed" Monte Carlo model respectively, compared to "TG-43sim" (Table 4.5 and Figure 4.9).

DVHs (Figures 4.8d,e) and local doses to voxels (Figure 4.8a) consistently show minor differences between "TG-43sim" and "Water" models which include interseed effects, with the largest differences occurring near seeds. The inclusion of interseed effects in the "Water" model introduces a shadowing effect of a few percent due to the increased attenuation in the seeds (Figure 4.8c). "Water" PTV $\mathrm{D}_{90}, \mathrm{~V}_{100}, \mathrm{~V}_{200}$ and skin $\mathrm{D}_{1 \mathrm{~cm}^{3}}$ are each found to be up to $4 \%, 1 \%, 5 \%$ and $4 \%$ lower, respectively, than "TG-43sim" due to interseed effects. DHI is found to be up to $5 \%$ higher with "TG-43sim" compared to "Water" because of the additional shadowing effect heterogeneities introduced by modelling the interseed effects. 

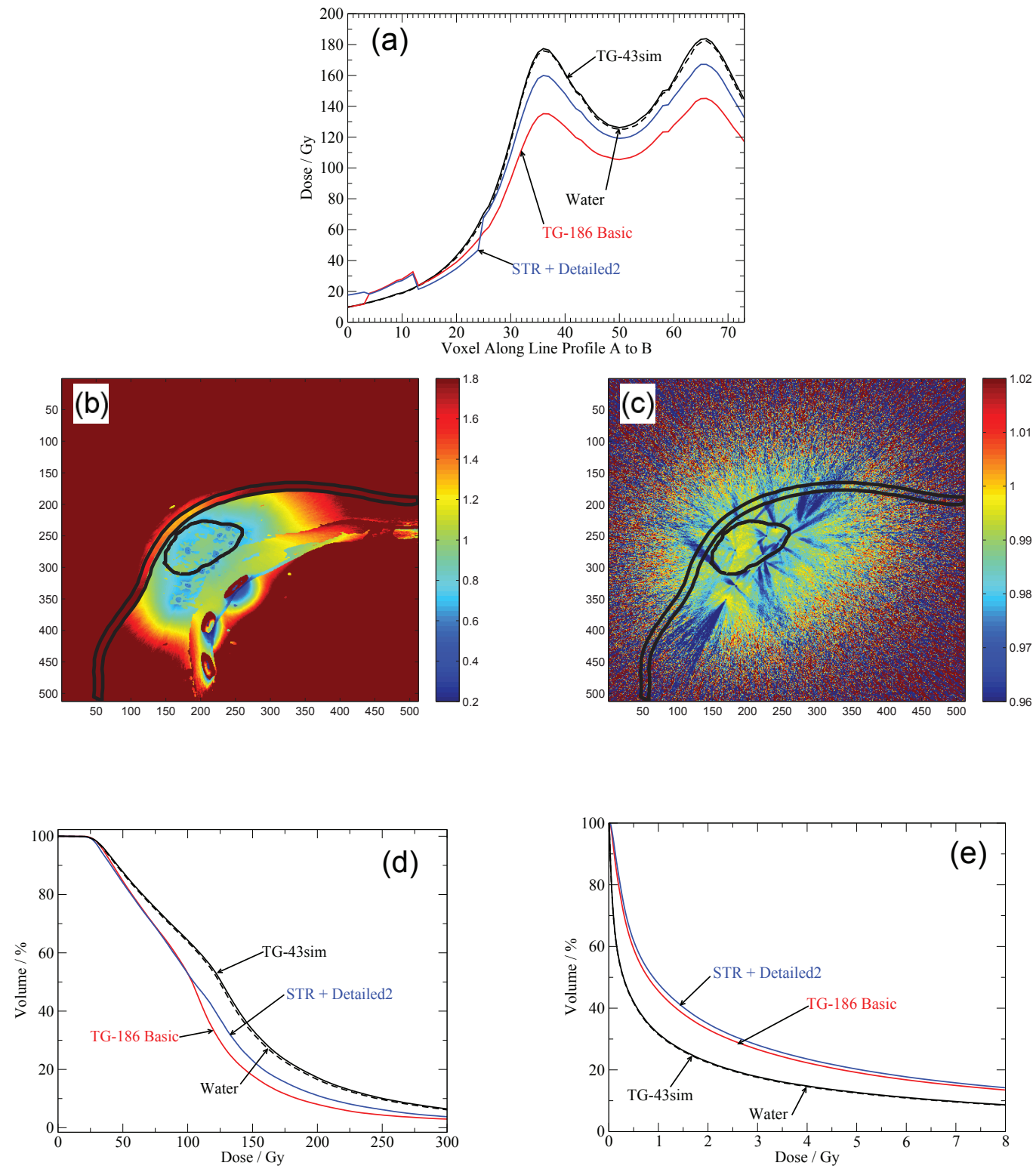

Figure 4.8: Calculated doses for one patient comparing "TG-43sim", "Water" (dashed line), "TG-186 Basic" and detailed Monte Carlo models. (a) Dose along the line profile shown in Figure 4.3. (b) "STR+Detailed2" / "TG-43sim" showing the overall difference between "TG-43sim" and one detailed Monte Carlo model. (c) "Water" / "TG-43sim" showing interseed effects. (d) PTV and (e) skin DVHs. 


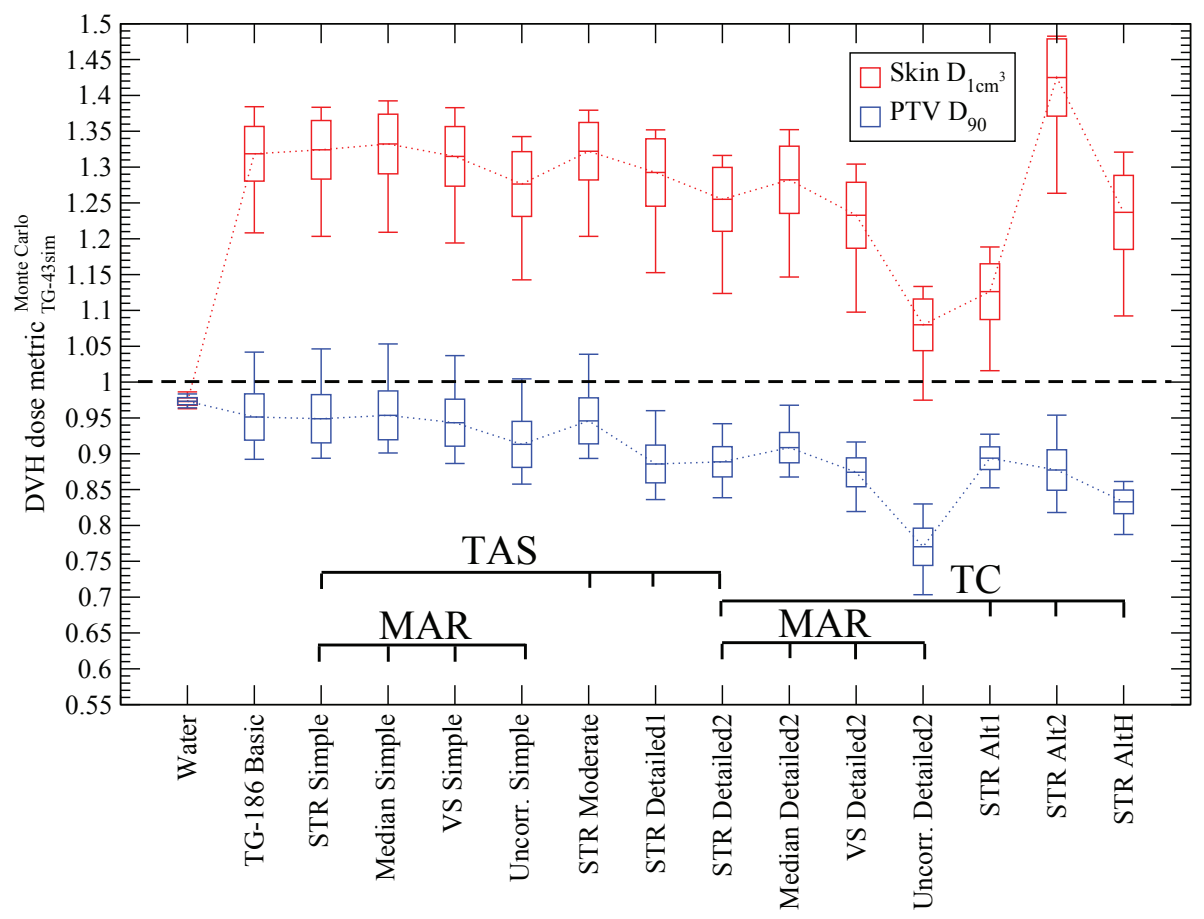

Figure 4.9: General trends in dose metrics (PTV D90 (blue) and skin $\mathrm{D}_{1 \mathrm{~cm}^{3}}$ (red)) calculated with various Monte Carlo models relative to "TG-43sim". Centre lines, box limits and whisker limits are mean, standard error on the mean and $\min / \max$ values of four patients respectively. The bold dashed line indicates $\mathrm{y}=1$, where Monte Carlo results would be equal to TG-43. "MAR", "TC" and "TAS" indicate Monte Carlo models which vary metallic artifact reduction method, tissue composition and tissue assignment scheme respectively.

\subsection{Discussion}

Monte Carlo dose calculations can produce a considerable range of results depending on the assumptions used to derive the virtual patient models. For breast permanent implant brachytherapy we have demonstrated varying levels of dependence on metallic artifact reduction, tissue assignment scheme and tissue elemental composition. Ultimately, these modelling assumptions introduce an inherent uncertainty in dose calculations. However, general trends between Monte Carlo and the standard 
TG-43 models are evident (Figure 4.9): "TG-43sim" overestimates dose to the target $\left(\mathrm{D}_{90}\right.$ on average by $10 \%$ and up to $\left.27 \%\right)$ and underestimates dose to the skin $\left(\mathrm{D}_{1 \mathrm{~cm}^{3}}\right.$ on average by $29 \%$ and up to $48 \%$ ) compared to post-MAR CT image derived virtual patient models. As clinics incorporate MBDCAs into the treatment planning process for breast permanent implant brachytherapy, the absolute dose prescribed to the PTV and dose threshold limits to the skin may increase to account for these general differences between doses computed with TG-43 and MC. The general trends between TG-43 and Monte Carlo models are consistent with other publications for various breast brachytherapy treatments. ${ }^{48,80,87}$

Specification of dose uniformity can further characterize dose distributions beyond the DVH metrics, and is considered useful even for highly heterogeneous interstitial implant distributions. ${ }^{93}$ The DHI computed for the "TG-43sim" models (0.17-0.37) are lower than those observed in a study of HDR breast brachytherapy (0.5-0.76), ${ }^{94}$ since the cases studied herein use ${ }^{103} \mathrm{Pd}$ sources which emit a photon energy spectrum about an order of magnitude less average energy than ${ }^{192}$ Ir resulting in much steeper dose gradients near the sources, yielding more heterogeneous PTV dose distributions. For the MC simulations of PBSI including non-water media considered herein, DHI values are always higher than those for "TG-43sim".

The differences between Monte Carlo and "TG-43sim" computed doses can be understood in terms of considerations of energy absorption and attenuation coefficients, and mass densities of the relevant tissues. In this work, the breast has been modelled as mainly adipose plus some gland tissue, both having $\mu_{e n} / \rho$ lower than water over the relevant energies (figure 4.1); mass densities assigned for adipose are less than unity. Compared with water, lower doses are computed in the sources' vicinity and there is a shallower dose gradient resulting in decreased total dose deposited inside the target volume which lowers $\mathrm{D}_{90}$ and the volume metrics (with MC compared 
to "TG-43sim"). Further, this causes a larger fractional reduction of the higher dose volume metrics (i.e., $\mathrm{V}_{200}$ compared to $\mathrm{V}_{100}$ ) which increases the Dose Homogeniety Index in the target volume in the MC models compared to "TG-43sim". The higher doses computed to the skin with MC compared to "TG-43sim" result from a compromise between the marginally lower $\mu_{e n} / \rho$ of skin compared to water, the reduced backscatter component in the MC models (due to modelling air outside the breast), and the relatively higher photon fluence reaching the skin in the MC models compared to "TG-43sim".

Post-implant CT images require metallic artifact mitigation since doses calculated using virtual patient models derived from uncorrected CT images can have large errors. Ideally, a MAR method should not affect artifact-free regions of the CT images, so, if a MAR method is constrained to image regions which contain artifacts, then seed position should be used to guide the MAR rather than target volume as sources may exist outside the contour (Figure 4.2a). This work demonstrates the mitigation of seed artifacts in the breast using various MAR methods, but each method has limitations which are general or specific to the breast. The STR, median filter and virtual sinogram MAR methods are each able to mitigate the bright spot artifacts around the seeds which are the most significant in terms of misassigned tissue in the virtual patient models leading to inaccuracies in dose calculations. The STR is unable to mitigate low CT number artifacts but it was not generally observed that a higher fraction of adipose (which is mapped to lower CT numbers than gland) was assigned compared to models derived using the median filter and virtual sinogram MAR methods (Table 4.3). The median filter smooths and blurs the image which has the potential to inhibit differentiation between adipose and gland: on average, a higher percentage of voxels were assigned to adipose for the median filter compared to STR and virtual sinogram (Table 4.3). The virtual sinogram method introduces new streaks but these streaks did not have a considerable effect on mean CT number 
(Figure 4.4) or relative assignment of adipose and gland in the virtual patient models (Table 4.3). Despite the differences in post-MAR CT images, the resulting DVHs and dose metrics are generally consistent $\left(\mathrm{D}_{90}\right.$ within $\left.6 \%\right)$ between the STR, median filter and virtual sinogram derived models (for the same tissue assignment scheme). Varying the MAR method causes considerable local dose differences when variations in CT number lead to differences in tissue assignment, which is more common with the "Detailed1" and "Detailed2" tissue assignment schemes. Overall, dose calculations are less sensitive to the MAR method than to tissue assignment scheme and tissue composition.

Various options are possible for assigning tissues in the virtual patient models, one of which is assuming the soft tissue in breast can be modelled as a homogeneous mixture of adipose and gland. The TG-186 recommended $80 \%$ adipose and $20 \%$ mammary gland, by mass, homogeneous mixed tissue (employed herein) is motivated by the work of Ref. ${ }^{86}$ who found the average, by volume, gland composition in the whole breast to be $13.7 \%$ - 25.6\% (mean 19.3\%). Other work ${ }^{48}$ reported a higher average mass fraction of gland in the breast PTV of $37.5 \%$, whereas we have modelled the breast tissue in the PTV to be $12-62 \%$ gland with the detailed segmented models (Table 4.1). Compared to the "Detailed2" models, the simpler mixed tissue models result in a $2 \%-13 \%$ higher PTV $D_{90}$ and a $1 \%-9 \%$ higher skin $\mathrm{D}_{1 \mathrm{~cm}^{3}}$.

The $80 \%$ adipose- $20 \%$ gland population-averaged tissue will not be representative of the breast composition for all patients due to the large inter-patient variability in relative adipose and gland content, so more realistic virtual patient models necessitate segmentation of these tissues. Differentiating between adipose and gland in post-implant $\mathrm{CT}$ images remains a challenge because the $\mathrm{CT}$ numbers of these tissues are similar, seed artifacts compromise the CT numbers near sources and the true relative fraction of these tissues for any given patient is unknown. In this work 
we used a consistent mass density threshold for all patients, based on the mid-way point between the theoretical nominal mass density of each tissue, and indeed this caused a significant variation in relative adipose and gland assignment between patients. We found a small adjustment of adipose/gland segmentation threshold (about $0.03 \mathrm{~g} / \mathrm{cm}^{3}$ ) between the "Detailed1" and "Detailed2" schemes caused variation in PTV and skin metrics of a few percent $\left(\mathrm{D}_{90}\right.$ up to $3 \%$ and $\mathrm{D}_{1 \mathrm{~cm}^{3}}$ up to $4 \%$ ) - suggesting any employed adipose and gland segmentation scheme should be clearly reported, carefully considered and justified.

There are a number of primary references for the elemental compositions of adipose, gland and skin, which vary significantly in mass energy absorption coefficients at low photon energies (Figure 4.1). This work shows that the choice of tissue composition can alter the target and skin DVHs and significantly change the target and skin dose metrics by up to tens of percent. This is consistent with previous breast modelling studies which also indicate a strong dependence on tissue composition, ${ }^{47,81}$ with results highly dependent on the tissues, geometries and dose scoring metrics.

In addition to the tissue modelling effects, Monte Carlo dose calculations are more accurate than those obtained under TG-43 conditions because they can consider realistic interseed scattering and attenuation. It has been previously reported that interseed effects in PBSI only contribute a few percent to the dose differences for ${ }^{103} \mathrm{Pd}^{80}$ and ${ }^{125} \mathrm{I}^{85}$ sources. This study is in agreement with the previous reports, suggesting that interseed effects influence dose calculations less than tissue modelling effects, and most dose variations are observed near seeds. The PTV $\mathrm{D}_{90}$ and skin $\mathrm{D}_{1 \mathrm{~cm}^{3}}$ were each lowered by up to $4 \%$ when including interseed effects in "Water" calculations.

In the current work, seeds are modelled as oriented along the CT axis ( $\mathrm{Su}-$ perior - Inferior), which does not include considerations for patient-dependent needle 
orientation during planning, post-implant swelling and edema as well as any patient misalignment with respect to the plan. A previous dose calculation study of ${ }^{125} \mathrm{I}$ prostate brachytherapy ${ }^{24}$ compared idealized and realistic seed orientations and reported no significant changes to target dose metrics, however, it is unclear whether this finding is applicable to more heterogeneous ${ }^{103} \mathrm{Pd}$ PBSI models. Since true seed orientation is not known for the patients studied herein, dose calculations were repeated with the "TG-43sim" and "STR+Detailed2" models but with seeds rotated either 90 degrees (most extreme rotation) or randomly rotated between zero and 45 degrees (more realistic seed orientation). In the PTV and the skin, dose metrics either increased or decreased depending on the patient, and the scale of dose differences was comparable between the "TG-43sim" and "STR+Detailed2" cases (i.e., seed orientation differences are not highly dependent on tissues or interseed effects). In the target, the largest difference was in $\mathrm{D}_{90}$ which differed by up to $4 \%$ and up to $20 \%$ with the random and 90 degree rotations respectively, compared to the axial seed alignment. In the skin, $\mathrm{D}_{1 \mathrm{~cm}^{3}}$ varied by up to $6 \%$ and up to $20 \%$, with the random and 90 degree rotations respectively, compared to the axial seed alignment. Despite this, Monte Carlo relative to "TG-43sim" results (with consistent seed orientations) showed the axial, random and 90 degree rotation models differed from each other by up to $2 \%\left(\mathrm{D}_{90}\right), 0 \%\left(\mathrm{~V}_{100}\right), 1 \%\left(\mathrm{~V}_{150}\right), 0 \%\left(\mathrm{~V}_{200}\right)$ and $4 \%\left(\mathrm{D}_{1 \mathrm{~cm}^{3}}\right)$. Although future work is needed to study seed orientation in PBSI models more rigorously, these results suggest that absolute dose calculations are sensitive, but dose ratios (MC relative to TG-43) are fairly insensitive to seed orientation modelling assumptions.

While the water-based TG-43 approach is inaccurate for breast permanent implant brachytherapy, it clearly offers consistency across clinical practice which risks being lost with the adoption of MBDCA-based approaches that rely on CT-derived virtual patient models. The results presented herein may be used to both motivate choices for a consensus approach and assess the uncertainties or variations due to 
different modelling assumptions, as well as inform future studies. Thus, in the following we present some considerations towards the development of a consensus approach which reinforce and build upon those presented in TG-186.

Voxelized patient-specific virtual models should be derived from post-implant CT scans and retain the voxel resolution of the CT to avoid CT number interpolation and averaging effects. CT images should be accompanied by physician-drawn contours which include (as a minimum) the target volume of clinical interest and the main organ-at-risk for PBSI, the skin; contouring guidelines should be consistent, e.g. the margin added around the implant bed and the thickness of the skin. Voxel mass density should be derived from CT number using, wherever possible, a scanner-specific CT number to mass density calibration since there can be considerable patient-topatient variation in breast density. Within images, bright spot artifacts due to seeds should be mitigated while maintaining the integrity of artifact-free regions of the CT images. The seed-guided threshold (STR) approach is favoured due to its simplicity in terms of implementation and its preservation of CT numbers near the artifacts, hopefully enabling differentiation of gland and adipose tissues. We demonstrated up to $6 \%$ variation in $\mathrm{PTV}_{90}$ and skin $\mathrm{D}_{1 \mathrm{~cm}^{3}}$ with MAR approach. Soft tissues within the breast (target volume) should be assigned separately as adipose or gland instead of an artificial mixed tissue derived from population-averaged compositions; we found PTV $D_{90}$ and skin $\mathrm{D}_{1 \mathrm{~cm}^{3}}$ to differ by up to $13 \%$ and $9 \%$, respectively, when modelling adipose and gland separately compared to a mixed-tissue model. The approach to differentiate between adipose and gland should be well-described and motivated: in the current work, a consistent CT number threshold was used for all patients based on the midway point between average adipose and gland densities reported by ${ }^{48}$ comparing these results ("Detailed2") to those with a different threshold ("Detailed1") based on adipose and gland density from ${ }^{42}$ changes $\mathrm{D}_{90}$ up to $3 \%$ and $\mathrm{D}_{1 \mathrm{~cm}^{3}}$ up to $4 \%$. Outside the target volume, the virtual patient model should be as accurate as 
possible, e.g. we additionally segmented lung, bone and the air (from soft tissue) for accuracy in scatter conditions (although sensitivity to these choices has not been explicitly studied herein). Until a confident approach of determining patient-specific tissue compositions is established, we suggest the use of consistent assumed elemental compositions for adipose, gland and skin (and other tissues), such as those listed by TG-186; assumed tissue composition affects PTV $\mathrm{D}_{90}$ and skin $\mathrm{D}_{1 \mathrm{~cm}^{3}}$ by up to $10 \%$ and $27 \%$ respectively.

Calcification modelling remains an outstanding issue for model-based dose calculations $^{47,84}$ and this challenge was not addressed in this study because patients were considered ineligible for treatment if calcifications were observed on post-surgical imaging. For these cases with no large calcifications in the breast tissue, it is possible that undetected microcalcifications are present which may be much smaller than the CT image voxel size resulting in slightly elevated CT numbers compared to soft tissue. A robust approach to model large calcifications and diffuse microcalcifications remains unclear and future work which systematically compares various modelling choices in carefully selected calcified and non-calcified cases should be performed.

\subsection{Conclusions}

Monte Carlo dose calculations have the potential to be more accurate than TG-43, but they rely on the derivation of accurate virtual patient models which require several necessary modelling steps and assumptions related to different possible metallic artifact reduction approaches, tissue assignment schemes of varying complexity and choice of tissue elemental compositions. Metallic artifact reduction should be employed for retrospective dose calculations which derive patient models from post-implant CT images, even if simple tissue assignment schemes are employed. Local doses are fairly 
insensitive to the type of MAR applied except where differences in CT number lead to differences in tissue assignment. The STR approach offers artifact mitigation comparable to more sophisticated approaches while still maintaining the possibility of differentiating between adipose and gland near the seeds. Virtual patient models which employ artificial mixed adipose and gland tissues with relative abundance based on population-averaged data may be inaccurate due to large inter-patient variability in breast composition. More detailed tissue assignment models which separately assign adipose and gland may be able to better account for this inter-patient variability but will be sensitive to the method of differentiating these tissues. Virtual patient models with segmented adipose/gland tissues can yield large differences in computed dose when alternate published tissue elemental compositions are employed. Despite the significant range of calculated doses originating from the assumptions used to derive the virtual patient models, TG-43 generally overestimates dose to the target and underestimates dose to the skin compared to Monte Carlo, and interseed effects play a minor role in these differences compared to tissue modelling effects. For consistency in clinical practice with the adoption of advanced model-based dose calculations, welldefined and consistent artifact mitigation approaches and tissue assignment protocols should be employed using justified and clearly-defined tissues (types and elemental compositions) assigned within each image structure and well-motivated mass density boundaries between tissues. 


\section{Chapter 5}

\section{Monte Carlo Dose Calculations for Prostate Brachytherapy}

This Chapter explores Monte Carlo dose calculations in a large cohort of permanent implant prostate brachytherapy patients. Comparisons are made between TG-43 and full tissue dose calculations for the target volume and several organs-at-risk. A sensitivity analysis is performed to explore how dose calculations are affected by method to model calcifications and the assumed elemental composition of calcification and organs-at-risk tissues. 


\subsection{Introduction}

Several Monte Carlo (MC) studies have explored how interseed scatter and attenuation effects (ISA) and modelling realistic tissues can influence permanent implant prostate brachytherapy (PIPB) dose calculations, ${ }^{54,78,81,91,95-98}$ and prostatic calcifications have been shown to significantly affect PIPB doses ${ }^{54,82,99,100}$ with dependence

on size, structure and location. ${ }^{101-103}$ However, existing publications are limited by studying small patient cohorts and are difficult to directly compare due to inconsistent simulation assumptions.

Towards the goal of clinically adopting MC dose calculations for brachytherapy, this Chapter presents a large scale dosimetry study. Using data from 613 patients treated at Centre Hospitalier Universitaire de Québec between 2003 and 2012, patient-specific MC and TG-43 simulations are carried out to enable a comprehensive dosimetric analysis. As dose calculations are sensitive to patient modelling assumptions, a sensitivity analysis with respect to assumed tissue elemental composition and calcification modelling approach is also presented.

\subsection{Methods and Materials}

\subsubsection{Patient Data}

Post-implant data from $613^{125} \mathrm{I}$ (Nucletron SelectSeed, ProstaSeed 125SL, Draximage LS-1 and OncoSeed 6711) (Chapter 1 Figure 1.1) PIPB patients are retrospectively studied (Table 5.1). Patient data include physician drawn target, urethra, rectum and bladder contours and additional calcification contours $\left(0.2-3.6 \mathrm{~cm}^{3}\right)$ were generated for a subset of patients. Post-implant CT images have a $(384-512) \times(384-512) \times$ 
$(31-81)$ voxel resolution corresponding to $(13.5-19.6) \times(14.2-19.6) \times(7.8-20.3) \mathrm{cm}$.

Table 5.1: Patient data characteristics: full cohort $(n=613)$ and the calcified $(n=25)$ and non-calcified $(\mathrm{n}=25)$ groups used for sensitivity analysis.

\begin{tabular}{|c|c|c|c|c|c|c|}
\hline $\begin{array}{l}\text { Patient } \\
\text { Group }\end{array}$ & $\begin{array}{c}\text { Number } \\
\text { of } \\
\text { Sources }\end{array}$ & $\begin{array}{c}\text { Air-Kerma } \\
\text { Strength } \\
{[\mathrm{U}]}\end{array}$ & $\begin{array}{c}\text { Target vol. } \\
\text { min,avg,max } \\
{\left[\mathrm{cm}^{3}\right]}\end{array}$ & $\begin{array}{l}\text { Urethra vol. } \\
\text { min,avg,max } \\
{\left[\mathrm{cm}^{3}\right]}\end{array}$ & $\begin{array}{l}\text { Rectum vol. } \\
\text { min,avg,max } \\
{\left[\mathrm{cm}^{3}\right]}\end{array}$ & $\begin{array}{c}\text { Bladder vol. } \\
\text { min,avg,max } \\
{\left[\mathrm{cm}^{3}\right]}\end{array}$ \\
\hline Full Cohort & $23-88$ & $0.43-0.85$ & $12,36,84$ & $0.2,0.7,2.0$ & $11,41,167$ & $2,33,104$ \\
\hline Calcified & $36-69$ & $0.74-0.84$ & $24,40,71$ & $0.3,0.7,1.8$ & $17,40,101$ & $15,33,50$ \\
\hline Non-Calcified & $34-76$ & $0.74-0.85$ & $21,38,65$ & $0.3,0.7,1.7$ & $20,41,79$ & $3,32,76$ \\
\hline
\end{tabular}

\subsubsection{Metallic Artifact Reduction}

CT artifacts are mitigated using a threshold approach: ${ }^{84}$ for voxels within $5 \mathrm{~mm}$ of seed centres, densities above $1.1 \mathrm{~g} / \mathrm{cm}^{3}$ are assigned to $1.04 \mathrm{~g} / \mathrm{cm}^{3}$. Voxels contained inside a calcification contour bypass the metallic artifact mitigation. The choice of artifact mitigation method may affect the target $\mathrm{D}_{90}$ by up to $6 \%$ (Chapter 3 ). ${ }^{84}$

\subsubsection{Virtual Patient Model}

Voxelized virtual patient models are derived from post-MAR CT images by mapping CT number to tissue elemental composition and mass density (Table 5.2). For all patients, two models are derived: a simulated TG-43 model which assigns all voxels to water with mass density $0.998 \mathrm{~g} / \mathrm{cm}^{3}$ and omits inter-seed effects (TG43sim) and a patient-specific model which assigns density and tissue-type by CT number and voxel location relative to contours (MCref). CT number to density calibration is the same as Chapter 3 Table 3.2, simulated tissue compositions are in Chapter 2 Tables 2.1 and 2.2, and corresponding mass energy absorption coefficients are in 
Figure 5.1. In addition to prostate and calcification, an artificial homogeneous mixed $50 \%$ prostate and $50 \%$ calcification tissue $e^{82,84}$ is modelled in the target to account for voxels which may encompass a boundary between calcification and prostate or contain micro-calcifications embedded in prostatic tissue.

\subsubsection{Sensitivity Analysis}

To explore the sensitivity of the MC dose calculations, simulations are repeated for 50 patients (25 with and 25 without visible prostatic calcifications) using alternate tissue compositions and calcification modelling approaches (described in Table 5.2).

Sensitivity to OAR tissue composition is explored by substituting MCref compositions with alternatives from the literature: alternate MC models, MCalt, for urethra, rectum and bladder are labelled $\mathbf{U} 1, \mathrm{U} 2, \mathbf{R} 1, \mathbf{R} 2, \mathbf{B} 1$ and $\mathbf{B} 2$, respectively. Sensitivity to calcification modelling approach is studied with modified versions of MCref. $\mathbf{P}$ assigns target voxels to Prostate, $\mathbf{P C}$ assigns voxels to Prostate or Calcification(Breast) and Mix assigns voxels to Prostate, Calcification(Breast) or one of four by-mass homogeneous prostate/calcification mixtures at $20 \%$ mass-fraction increments. Additionally, a similar version of PC, labelled PCC, assigns all target voxels as Prostate except those within calcification contours as Calcification(Breast) (i.e., calcifications are delineated by contour rather than CT number). Sensitivity to calcification tissue composition is explored in the 25 patients with calcifications. Four alternate versions of the $\mathbf{P C}$ scheme, PC-1,2,3,4, assign target voxels as Prostate or one of four recently published prostatic calcification compositions Calcification$1,2,3,4 \cdot{ }^{103}$ 

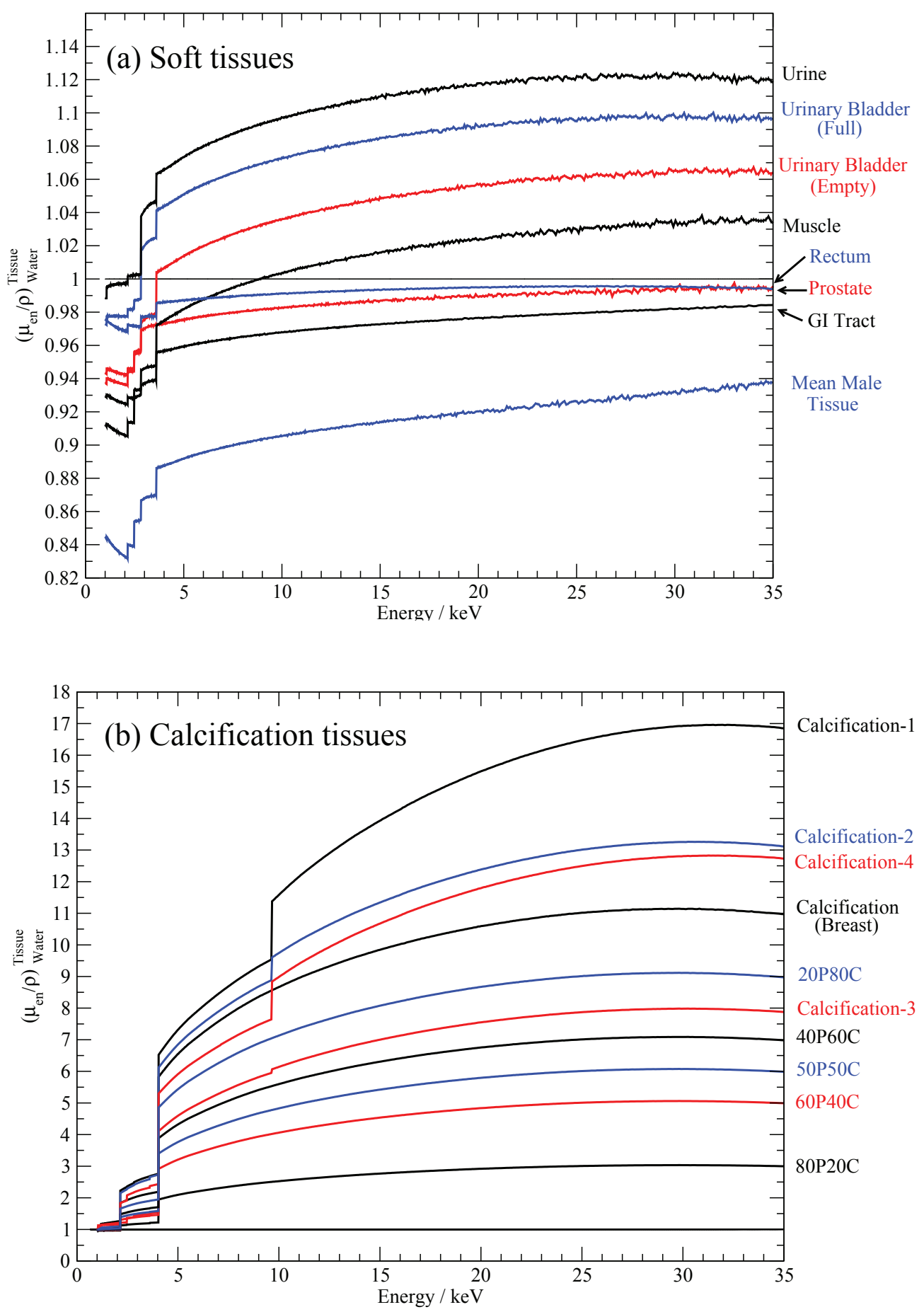

Figure 5.1: Mass energy absorption coefficients, normalized to water, for the Monte Carlo simulated tissues with compositions described in Tables 2.1 and 2.2. The major photon emission peaks for ${ }^{125} \mathrm{I}$ are $27.2,27.5,31.0,31.7$ and $35.5 \mathrm{keV} .{ }^{20}$ 
Table 5.2: Tissue assignment schemes for the reference MC model, MCref, and the alternate MC models, MCalt, for the sensitivity analysis. Elemental compositions and effective atomic number for simulated tissues are in Tables 2.1 and 2.2.

\begin{tabular}{|c|c|c|}
\hline \multicolumn{3}{|c|}{ Reference MC Model "MCref" } \\
\hline Region & Tissues & Mass Density Range \\
\hline \multirow[t]{3}{*}{ Target } & Prostate $^{42}$ & $\leq 1.14 \mathrm{~g} / \mathrm{cm}^{3}$ \\
\hline & $50 \mathrm{P} 50 \mathrm{C}$ & $1.14-1.27 \mathrm{~g} / \mathrm{cm}^{3}$ \\
\hline & Calcification(Breast) ${ }^{45}$ & $>1.27 \mathrm{~g} / \mathrm{cm}^{3}$ \\
\hline Urethra & Prostate & All \\
\hline Rectum & Rectum $^{44}$ & All \\
\hline Bladder & Urinary Bladder(Empty) $)^{42}$ & All \\
\hline \multirow[t]{2}{*}{ Remainder } & Mean Male Soft Tissue ${ }^{45}$ & $\leq 1.14 \mathrm{~g} / \mathrm{cm}^{3}$ \\
\hline & Cortical Bone ${ }^{42}$ & $>1.14 \mathrm{~g} / \mathrm{cm}^{3}$ \\
\hline
\end{tabular}

Sensitivity Analysis: Alternate Organ-at-Risk Tissues

\begin{tabular}{ll}
\hline MCalt & Difference from MCref model \\
\hline U1 & All urethra voxels as Muscle \\
U2 & All urethra voxels as Mean Male Soft Tissue \\
R1 & All rectum voxels as Muscle \\
R2 & All rectum voxels as GI Tract ${ }^{42,45}$ \\
B1 & All bladder voxels as Urinary Bladder $(\text { Full })^{42}$ \\
B2 & All bladder voxels as Urine \\
\hline
\end{tabular}

Sensitivity Analysis: Alternate Calcification Modelling Approach

\begin{tabular}{ll}
\hline MCalt & Difference from MCref model \\
\hline $\mathbf{P}$ & All target voxels as Prostate \\
$\mathbf{P C}$ & All target voxels as Prostate or Calcification(Breast) \\
& Delineated by $\left[1.14 \mathrm{~g} / \mathrm{cm}^{3}\right]$ \\
PCC & All target voxels as Prostate, except those in calcification \\
& contour as Calcification(Breast) \\
Mix & All target voxels as Prostate, mixed $20 \%$ increment $\mathrm{P} / \mathrm{C}$ tissue \\
& or Calcification(Breast), delineated by $\left[1.10,1.23,1.41,1.65,1.99 \mathrm{~g} / \mathrm{cm}^{3}\right]$ \\
\hline & \\
Sensitivity & Analysis: Alternate Calcification Composition \\
\hline MCalt & Difference from PC model \\
\hline PC 1-4 & All target voxels as Prostate or Calcification 1-4 103 \\
& Delineated by $\left[1.14 \mathrm{~g} / \mathrm{cm}^{3}\right]$ \\
\hline \hline
\end{tabular}




\subsubsection{Monte Carlo Dose Calculations}

EGSnrc $^{60,92}$ user-code BrachyDose ${ }^{57,58}$ is employed for dose calculations in voxelized virtual patient models using the same dose scoring grid as the CT images. Dose to the local voxel medium $\left(\mathrm{D}_{m, m}\right)$ is reported. Monte Carlo simulation parameters are consistent with those described in Ref. ${ }^{84}$ and Chapter 2. Statistical uncertainties to volumes are evaluated as the quadrature sum of the voxel uncertainties within each contour considering only voxels which receive at least $25 \%$ of the prescription dose. ${ }^{26}$ The simulation for each patient initializes $10^{9}$ particles resulting in mean (maximum) statistical uncertainty to the target, rectum, bladder and urethra contoured volumes of $0.18(0.25), 0.34(0.48), 0.35(0.44), 0.20(0.29) \%$ respectively. Achieving these statistical uncertainties required 9-15 CPU core hours per calculation, but obtaining target uncertainties of $\sim 2 \%$ would require $\sim 1$ min per patient on a multi-core processor (6-9 core minutes total).

The percent difference, $\% \Delta$, between two MC models, $M C_{1}, M C_{2}$, for a particular DVH dose metric is reported as,

$$
\% \Delta\left(M C_{1}, M C_{2}\right)=\frac{M C_{1}-M C_{2}}{M C_{1}} \times 100 \% .
$$

For example, $\mathrm{D}_{90} \% \Delta$ (MCref,TG43sim) is the percent difference between the $\mathrm{D}_{90}$

evaluated with MCref and TG43sim. Although ${ }^{125} \mathrm{I}$ brachytherapy dose distributions are characteristically heterogenous, the target volume dose homogeneity index (DHI) may be used to describe the quality of an implant: ${ }^{93}$

$$
D H I=1-\frac{V_{150}}{V_{100}} .
$$




\subsection{Results}

\subsubsection{Large Patient Cohort}

Across the 613 patient cohort, MCref and TG43sim average DVHs are similar to each other for each of the target, urethra, rectum and bladder, with MCref curves being a few percent lower. Large inter-patient variation in DVHs are calculated using both MCref and TG43sim (MCref ranges in Figure 5.2).
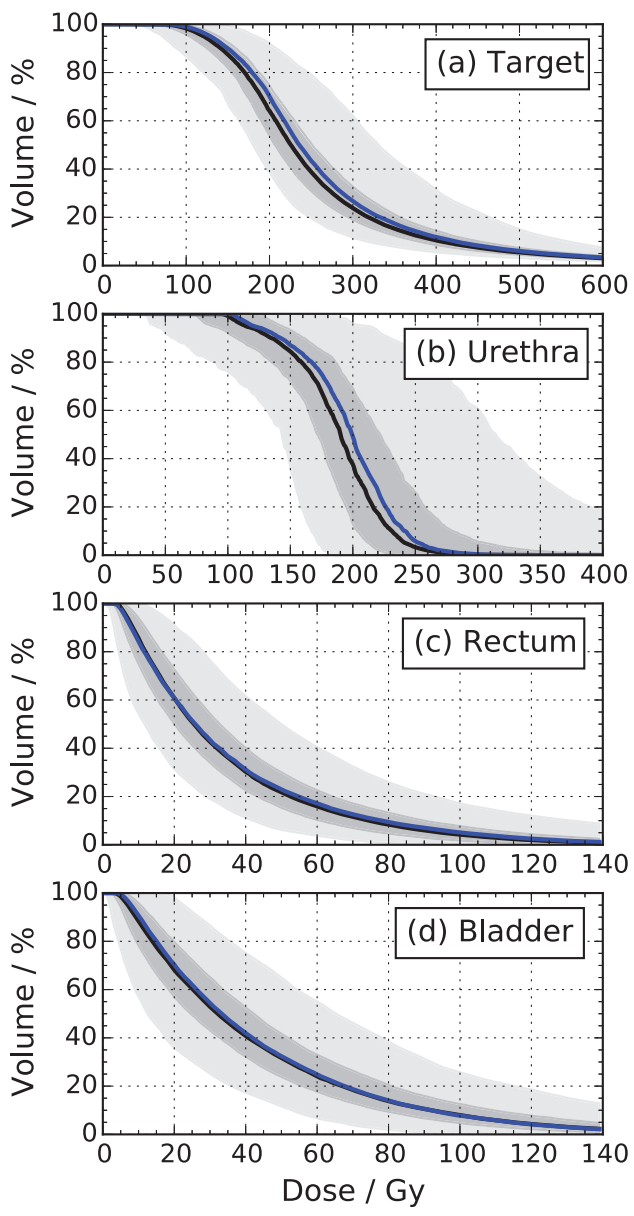

Figure 5.2: (a) Target (b) Urethra (c) Rectum and (d) Bladder average DVHs for 613 patients calculated using MCref (black) and TG43sim (blue). 50\% (90\%) of patients have MCref DVHs in the dark (light) grey-shaded area. 
In each contoured volume, the average $\% \Delta$ (MCref,TG43sim) across all patients $\left(\% \Delta_{a v}\right)$ for a given DVH metric is several percent, has a standard deviation of a few percent $\left(\% \Delta_{s t d}\right)$ and varies between metrics (Table 5.3). In the target, $\% \Delta_{a v}($ MCref,TG43sim) can be in excess of $-10 \%$. In the OARs, MCref and TG43sim metrics are generally within a few percent of each other, on average.

Figure 5.3 illustrates local dose differences in three cases: a patient with a large calcification (example 1), an intermediate sized calcification (example 2) and no visible calcification (example 3). Local dose differences due to inter-seed effects in the target and beyond are often more than 15\% (lower doses in MCref). In patients with calcifications, MCref yields relatively higher doses to calcifications and lower doses (occasionally by over $50 \%$ ) to regions surrounding the calcifications which may correspond to a clinically-relevant under-dosed volume. Abrupt changes in voxel $\% \Delta$ exist at the boundary of each contoured volume (target, rectum, bladder) and tissue heterogeniety (calcification, bone) due to the change in mass energy absorption coefficients between the simulated tissues. The differences in MCref and TG43sim dose metrics for these example cases are in Table 5.3.

Out of the 613 PIPB patients, $10.6 \%$ have visually identified prostatic calcifications. Calcifications decrease the target $\mathrm{D}_{90}$ and $\mathrm{V}_{100}$ by several percent, with higher target calcification fractions having the largest differences (Figure 5.4a). Generally, target $\mathrm{D}_{90}$ calculated with MCref and TG43sim are well correlated, however, outliers exist and are generally patients with larger calcifications (Figure 5.4b).

Dose homogeneity varies considerably across the 613 patients (MCref: 0.13 0.58, TG43sim: $0.11-0.54$ in $95 \%$ of patients). DHI is on average $12 \%$ higher with MCref than TG43sim, indicating TG-43 overestimates heterogeneity despite modelling the entire volume as uniform density water. Highly heterogeneous target dose distributions (low DHI) correlate with higher dose metrics in the target and urethra 
Table 5.3: Dose metrics evaluated with MCref and TG43sim for 613 patients and three example cases (Figure 5.3). $\% \Delta$, $\% \Delta_{a v}$ and $\% \Delta_{s t d}$ are individual patient, population average and standard deviation of percent differences between MCref and TG43sim metrics. IQR is the interquartile range of MCref doses.

\begin{tabular}{|c|c|c|c|c|c|c|c|c|c|c|c|c|c|}
\hline & \multicolumn{4}{|c|}{ Target } & \multicolumn{3}{|c|}{ Urethra } & \multicolumn{3}{|c|}{ Rectum } & \multicolumn{3}{|c|}{ Bladder } \\
\hline & $\begin{array}{l}\mathrm{D}_{90} \\
{[\mathrm{~Gy}]} \\
\end{array}$ & $\begin{array}{c}\mathrm{D}_{99} \\
{[\mathrm{~Gy}]} \\
\end{array}$ & $\begin{array}{l}\mathrm{V}_{100} \\
{[\%]}\end{array}$ & $\begin{array}{l}\mathrm{V}_{200} \\
{[\%]} \\
\end{array}$ & $\begin{array}{c}\mathrm{D}_{5} \\
{[\mathrm{~Gy}]} \\
\end{array}$ & $\begin{array}{c}\mathrm{D}_{30} \\
{[\mathrm{~Gy}]} \\
\end{array}$ & $\begin{array}{l}\mathrm{V}_{100} \\
{[\%]} \\
\end{array}$ & $\begin{array}{c}\mathrm{D}_{0.1 \mathrm{~cm}{ }^{3}} \\
{[\mathrm{~Gy}]}\end{array}$ & $\begin{array}{c}\mathrm{D}_{2 \mathrm{~cm}}{ }^{3} \\
{[\mathrm{~Gy}]} \\
\end{array}$ & $\begin{array}{l}\mathrm{D}_{30} \\
{[\mathrm{~Gy}]} \\
\end{array}$ & $\begin{array}{c}\mathrm{D}_{0.1 \mathrm{~cm} \mathrm{~m}^{3}} \\
{[\mathrm{~Gy}]}\end{array}$ & $\begin{array}{c}\mathrm{D}_{5} \\
{[\mathrm{~Gy}]} \\
\end{array}$ & $\begin{array}{l}\mathrm{D}_{30} \\
{[\mathrm{~Gy}]} \\
\end{array}$ \\
\hline \multicolumn{14}{|c|}{ Overall results from 613 patients } \\
\hline MCref & 144.1 & 94.6 & 88.2 & 30.0 & 271.4 & 222.2 & 83.4 & 176.3 & 97.5 & 42.8 & 221.8 & 120.1 & 54.9 \\
\hline TG43sim & 152.6 & 101.3 & 90.4 & 33.4 & 283.4 & 232.8 & 86.0 & 185.6 & 102.8 & 44.2 & 219.2 & 119.7 & 56.0 \\
\hline$\% \Delta_{a v}$ & -5.9 & -7.2 & -2.6 & -11.5 & -4.4 & -4.7 & -5.7 & -5.2 & -5.4 & -3.2 & 1.3 & 0.4 & -2.1 \\
\hline$\% \Delta_{s t d}$ & 1.6 & 2.5 & 1.7 & 3.2 & 1.8 & 1.9 & 6.5 & 1.8 & 1.7 & 5.3 & 1.8 & 1.5 & 2.0 \\
\hline IQR & 34.9 & 32.2 & 9.8 & 14.7 & 93.2 & 56.6 & 19.8 & 73.6 & 34.8 & 16.7 & 99.8 & 38.6 & 22.0 \\
\hline \multicolumn{14}{|c|}{ Example Case 1: $1.52 \mathrm{~cm}^{3}$ calcification } \\
\hline MCref & 110.4 & 72.4 & 73.3 & 19.7 & 230.8 & 182.6 & 79.1 & 121.4 & 64.6 & 25.1 & 224.6 & 97.9 & 34.9 \\
\hline TG43sim & 137.4 & 98.4 & 87.6 & 22.7 & 267.3 & 223.8 & 93.2 & 152.5 & 81.8 & 31.8 & 229.2 & 99.7 & 38.7 \\
\hline$\% \Delta$ & -24.4 & -35.9 & -19.4 & -15.5 & -15.8 & -22.6 & -17.8 & -25.6 & -26.6 & -26.6 & -2.1 & -1.8 & -10.9 \\
\hline \multicolumn{14}{|c|}{ Example Case $2: 0.32 \mathrm{~cm}^{3}$ calcification } \\
\hline MCref & 84.9 & 54.5 & 61.7 & 13.6 & 190.3 & 168.7 & 59.8 & 86.9 & 54.4 & 21.7 & 86.2 & 45.2 & 19.3 \\
\hline TG43sim & 92.9 & 60.5 & 68.4 & 15.2 & 203.7 & 183.3 & 68.4 & 94.1 & 58.6 & 23.5 & 87.0 & 44.4 & 20.6 \\
\hline$\% \Delta$ & -9.5 & -11.0 & -10.8 & -12.1 & -7.1 & -8.6 & -14.4 & -8.3 & -7.6 & -8.5 & -0.9 & 1.9 & -7.0 \\
\hline \multicolumn{14}{|c|}{ Example Case 3: No visible calcification } \\
\hline MCref & 114.2 & 73.5 & 79.1 & 20.5 & 193.6 & 162.0 & 59.0 & 99.4 & 62.4 & 27.2 & 247.0 & 141.1 & 70.4 \\
\hline TG43sim & 120.9 & 78.4 & 82.0 & 22.6 & 201.2 & 167.3 & 62.0 & 105.0 & 66.7 & 29.5 & 254.6 & 141.3 & 71.9 \\
\hline$\% \Delta$ & -5.9 & -6.8 & -3.6 & -10.1 & -4.0 & -3.3 & -5.2 & -5.6 & -6.9 & -8.4 & -3.1 & -0.1 & -2.1 \\
\hline
\end{tabular}


volumes (Figure 5.4c).

\subsubsection{Sensitivity Analysis}

Sensitivity of MC dose calculations to simulated tissue composition and calcification modelling approach assumptions are presented in Figure 5.5 for select DVH dose metrics. Modelling alternate urethra, rectum and bladder tissues (Figure 5.5a-d) results in negligible differences for target doses. However, substituting alternate elemental compositions within the organs-at-risk changes doses proportionally to mass energy absorption coefficients of the substituted tissues.

For non-calcified patients, the calcification modelling approach has marginal effect on doses (Figure 5.5e-h), except with Mix since a lower density threshold differentiates prostate tissue from mixed tissues causing some voxels which are assigned as Prostate with MCref to be assigned to 80P20C.

Considerable differences to target DVH metrics occur in calcified patients when modifying the calcification modelling approach (Figure 5.5e-h). Decreasing the amount of modelled calcification increases dose metrics: $\mathbf{P}$ increases the $\mathrm{D}_{90}$ by $4.2 \%$ on average, and Mix increases $\mathrm{D}_{90}$ by $1.3 \%$ on average since a higher fraction of bulk calcification is modelled as a mixed tissue. Simplifying the model by assigning only pure prostate or calcification tissue, $\mathbf{P C}$, decreases the $\mathrm{D}_{90}$ by $1.2 \%$ on average since the voxels modelled as 50P50C in MCref are modelled as Calcification(Breast) with PC. Similar doses are calculated when differentiating between Calcification(Breast) and Prostate using mass density (PC) or calcification contours (PCC). Modifying PC with alternate calcification compositions (PC-1,2,3,4 employing Calcification$1,2,3,4)$ can vary the target $\mathrm{D}_{90}$ by a few percent in calcified patients (Figure 5.5i-1). 

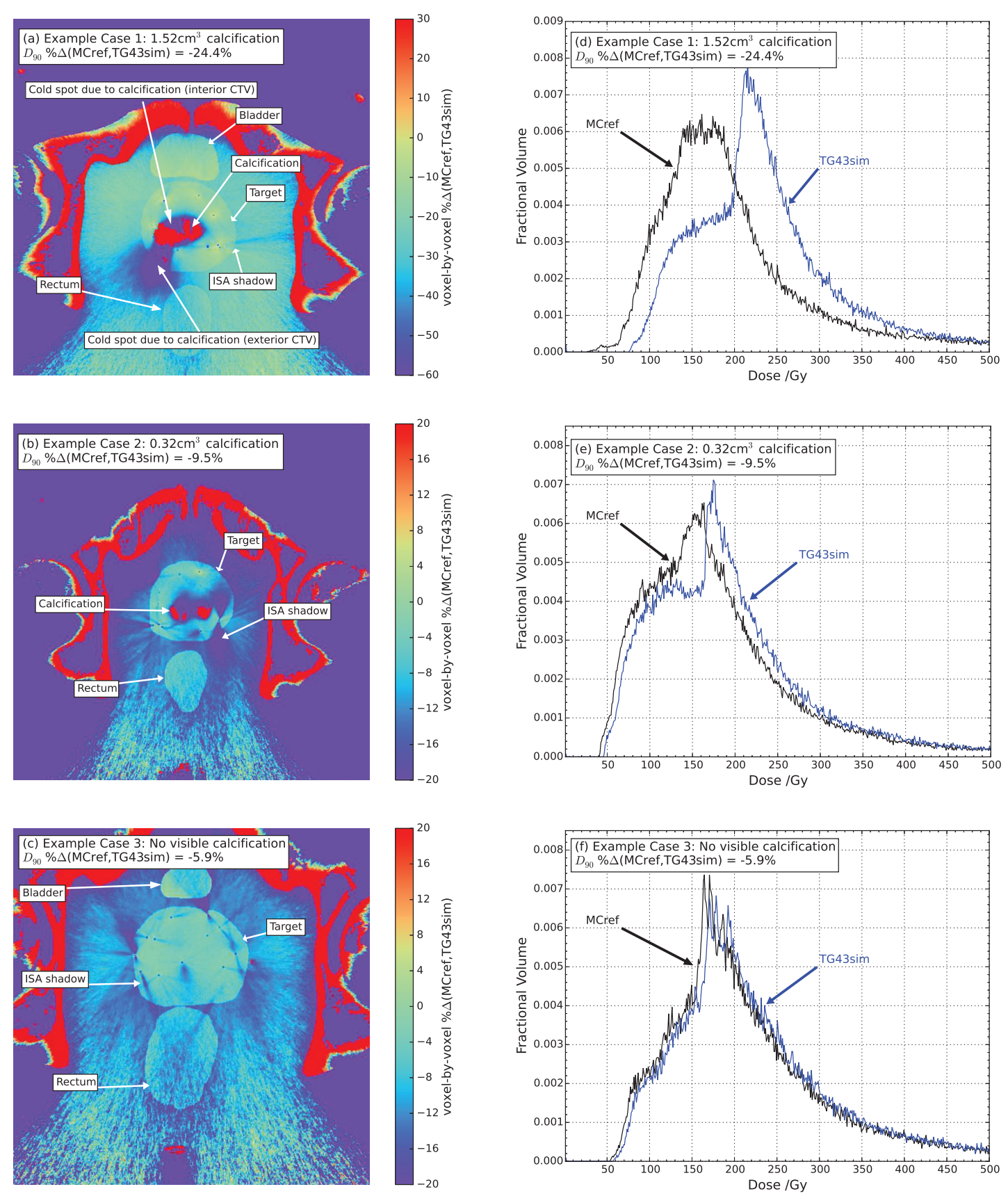

Figure 5.3: Three example patients with (a,d) large-sized, (b,e) intermediate-sized and $(c, f)$ no visible calcifications. (a-c) Voxel-by-voxel percent difference of dose with positive (negative) values indicating that doses are higher (lower) with MCref compared to TG43sim. (d-f) Corresponding differential DVHs for MCref and TG43sim. 

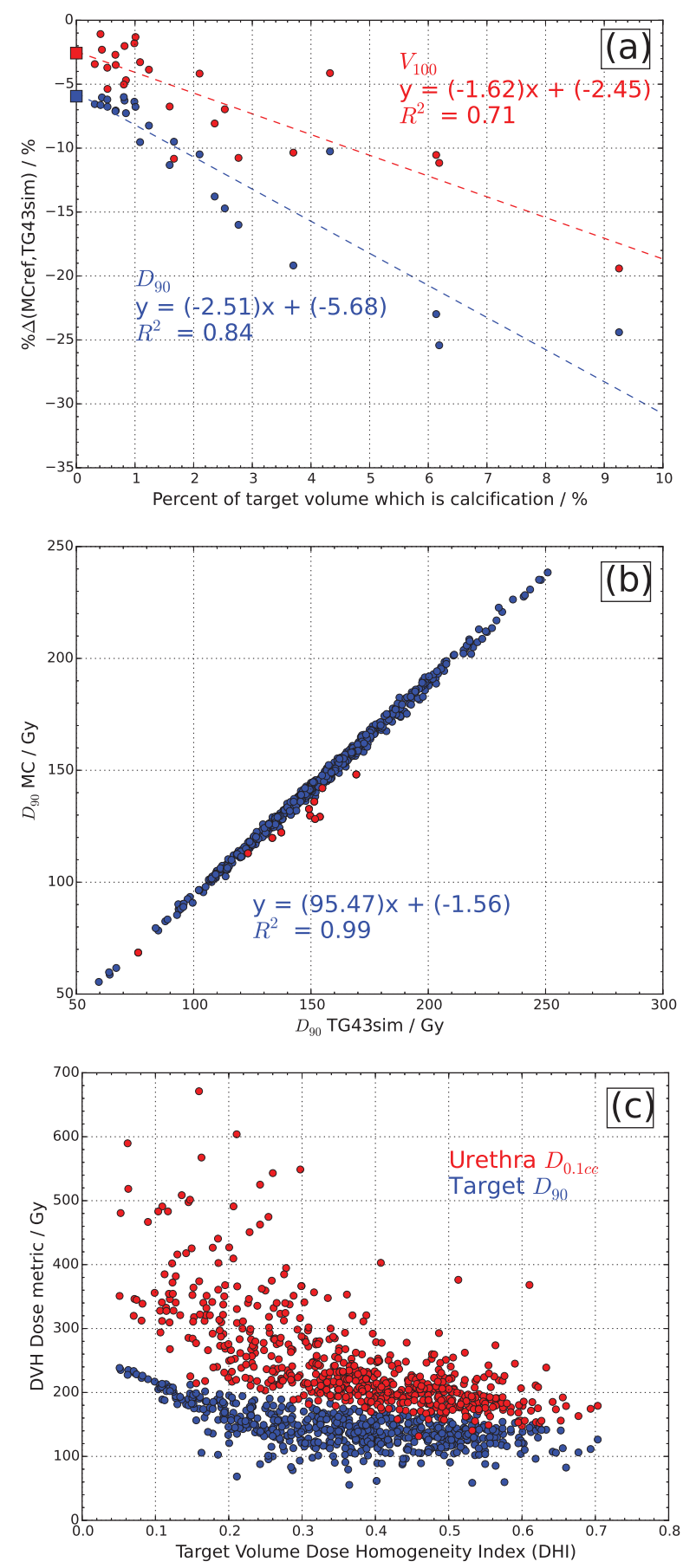

Figure 5.4: (a) Relationship between percentage of the target volume containing calcification (for the 25 calcified patients) and $\% \Delta$ (MCref,TG43sim) for $\mathrm{D}_{90}$ (blue) and $\mathrm{V}_{100}$ (red). Square points are $\% \Delta$ (MCref,TG43sim) for all 613 patients which closely match the fitted y-intercepts. (b) 613 patient TG43sim vs MCref $\mathrm{D}_{90}$ (blue) and outliers $(\% \Delta \geq 9 \%$ ) (red) which are patients with visible prostatic calcifications. (c) 613 patient target volume DHI (Equation 5.2) related to target $\mathrm{D}_{90}$ (blue) and urethra $D_{0.1 c c}$ (red) showing an increase in metrics for heterogeneous dose distributions. 

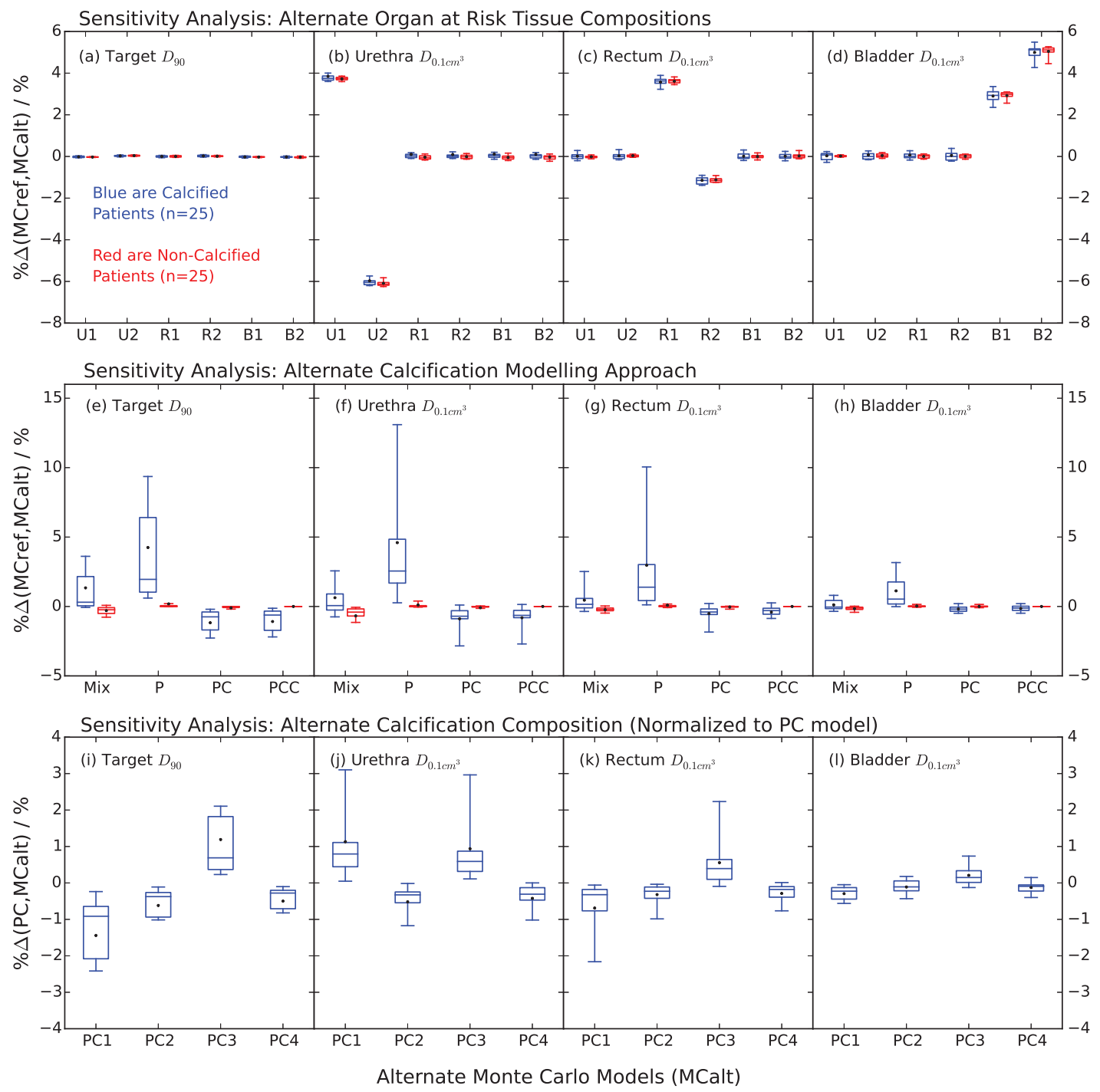

Figure 5.5: Sensitivity analysis of MC dose calculations to modelling assumptions. Box plots (mean point, median line, 50th percentile box, 95th percentile whiskers) of the percent difference, $\% \Delta$, between the alternate MC models (listed on $\mathrm{x}$-axis) and (a-h) MCref or (i-l) PC for select target, urethra, rectum and bladder dose metrics. 


\subsection{Discussion}

This work motivates the adoption of MC dose calculations for PIPB by demonstrating an average difference between MCref and TG43sim target and OAR dose metrics of several percent across a large patient cohort $\left(\% \Delta_{a v}\right.$ in Table 5.3). These average differences cannot be applied as correction factors to an individual patient as there is a considerable variation in $\% \Delta$ between patients $\left(\% \Delta_{s t d}\right.$ in Table 5.3$)$, e.g. target $\mathrm{D}_{90}$ $\left(\mathrm{V}_{100}\right) \% \Delta_{s t d} / \% \Delta_{a v}$ is $27 \%(65 \%)$. AAPM TG-43U1 cannot account for decreases in relative dose metrics due to calcifications (Figure 5.4a,b and Example cases 1,2 in Table 5.3) and lower local doses due to calcifications and inter-seed effects (Figure $5.3)$.

The results of the large scale dosimetry study between TG43sim and MCref are generally consistent with other work, but direct comparison is limited by differences in $\mathrm{MC}$ modelling approaches between studies. The target $\mathrm{D}_{90}$ and $\mathrm{V}_{100}$ $\% \Delta_{a v}$ (MCref,TG43sim) of $-5.9 \%$ and $-2.6 \%$ are comparable to reported -3 to $-13 \%$ for $\mathrm{D}_{90}$ and -2 to $-5 \%$ for $\mathrm{V}_{100} \cdot{ }^{78,82,96,99}$ Urethra $D_{5} \% \Delta_{a v}$ (MCref,TG43sim) of $-4.4 \%$ is similar to other publications (-3 to $-5 \%$ for $D_{10}$ and $-2 \%$ for $\left.D_{5}{ }^{78,82,99}\right)$. However, the rectum $D_{2 \mathrm{~cm}^{3}} \% \Delta_{a v}$ (MCref,TG43sim) of $-5.4 \%$ is smaller than published values of -11 to $-12 \% .^{99}$ Future work to correlate treatment outcomes with these dosimetric findings will build upon previous outcome reports ${ }^{66,79,104}$ and may motivate revision of PIPB prescription dose and OAR thresholds.

The sensitivity analysis indicates variability in MCalt doses are of similar order as patient-cohort average dose differences between TG43sim and MCref. This motivates the development of a well justified consensus PIPB modelling approach such as MCref. Sensitivity to prostate tissue composition was not performed but two alternate compositions are worth noting: ICRP89 prostate composition ${ }^{46}$ 
is equal to Mean Male Soft Tissue, and ICRP23 prostate composition ${ }^{44}$ has been shown to be dosimetrically equivalent to Prostate. ${ }^{81}$ Other studies have modelled homogeneous mixed prostate and calcification tissue $(\mathrm{s})^{82,84}$ but have relied on the uncertain nominal breast calcification mass density of $3.06 \mathrm{~g} / \mathrm{cm}^{3}$ to differentiate tissues, often resulting in few, if any, voxels assigned to pure calcification. On the other hand, MCref differentiates mixed tissues using the median of imaged calcification CT numbers which are much lower than the nominal density (mean (max) calcification density of $\left.1.3(2.4) \mathrm{g} / \mathrm{cm}^{3}\right)$. Contouring calcifications (PCC in Figure 5.5e-h) rather than using density to differentiate tissues (PC) has little effect on dose, however, contours can be useful to improve metallic artifact reduction by identifying voxels to skip which better preserves calcifications near sources. Modelling calcification as Calcification(Breast) yields "middle-of-the-pack" dose calculations compared to models assuming Calcification1-4 (Figure 5.5i-1). This suggests that Calcification(Breast) may continue to be a useful surrogate composition until a more definitive consensus prostate calcification composition is known. The four single-sample prostatic calcification compositions ${ }^{103}$ assume all light elements are oxygen, but modified Calcification1-4 compositions (results not shown) which replace the assumed oxygen content with $\mathrm{H}, \mathrm{N}, \mathrm{C}, \mathrm{O}$ in the same proportions as Calcification(Breast) generally do not affect $Z_{e f f}$, mass energy absorption coefficients or dose calculations. The ICRP $89^{46}$ urethra composition (same as Mean Male Soft Tissue) may unrealistically underestimate dose (Figure 5.5b U2) as a result of its lower mass energy absorption coefficient due to its $18 \%$ adipose content. ${ }^{43}$

Target DVH included all voxels within the target contour except those within the urethra contour. For the 50 patients included in the sensitivity analysis, target DVHs were re-derived using alternate assumptions: additionally including urethra voxels caused the target $\mathrm{D}_{90}$ and $\mathrm{V}_{100}$ to differ by less than $0.1 \%$ on average, and excluding doses to calcification voxels in patients with calcifications decreased $\mathrm{D}_{90}$ 
and $\mathrm{V}_{100}$ by $0.3 \%$ on average.

This study considers urethra, bladder and rectum as OARs, but future work exploring doses to penile bulb, neurovascular bundles and bladder neck may be clinically relevant. ${ }^{6,105} \mathrm{MC}$ dose calculations may be improved with dynamic modelling to account for prostate edema, ${ }^{106,107}$ which may affect target $\mathrm{D}_{90}$ by up to 15 Gy. ${ }^{108}$ Tissue differentiation remains a challenge for developing patient models since CT images provide limited soft tissue contrast, but dual energy x-ray or MR imaging may offer improvements. ${ }^{5,109-112}$ Future work is needed to apply MC dose calculations to PIPB radiobiological models to explore how they are influenced by ISA and dose perturbations from calcifications.

\subsection{Conclusions}

PIPB dose calculations in the target and OAR are on average lower in MC compared to TG-43 models by several percent with dose differences varying considerably between patients. Largest dose differences are found in patients with prostatic calcifications. MC dose calculations have a several percent dependence on modelling assumptions; OAR doses depend on OAR tissue composition and target doses in calcified patients depend on the calcification modelling approach. To assure consistency of practice, a consensus PIPB MC modelling approach such as MCref described herein, should be adopted in future studies. 


\section{Chapter 6}

\section{Radiobiological Modelling for Prostate Brachytherapy}

This Chapter couples the Monte Carlo dose calculations for the large cohort of PIPB patients, presented in the previous Chapter, to several existing PIPB radiobiological models. New challenges are identified, and general solutions proposed, related to enabling radiobiological models to account for dose heterogeneities which may result from tissue heterogeneities in the virtual patient model. Comparisons are made between biological dose and TCP estimates resulting from adopting different radiobiological models and incorporating water-based or full tissue model dose calculations. 


\subsection{Introduction}

The physical dose distribution remains the primary metric of treatment quality, but

radiobiological indices are of increasing clinical interest. ${ }^{25,66}$ Although radiobiological modelling may provide insight by accounting for biological response to radiotherapy, these models are limited by their requirement to assume particular mechanistic forms and rely on several largely uncertain biological and patient-specific parameters. In an attempt to establish a consensus approach, AAPM Task group report $137^{66}$ presented a detailed summary of numerous PIPB radiobiological models and recommended parameter values.

The purpose of this Chapter is to retrospectively compare, for a large group of PIPB patients, the biological doses and tumour control probabilities evaluated using Monte Carlo dose calculations representing TG-43 conditions or realistic tissue and interseed effects. Since radiobiological models vary considerably across the literature, several published models of varied complexity are considered using a consistent set of biological parameters. Additionally, new issues are identified related to incorporating dose calculation models which consider prostatic calcifications and suggest extensions to enable existing models to better account for dose perturbations resulting from tissue heterogeneities.

\subsection{Methods}

\subsubsection{Radiobiological models}

The biological dose attempts to model tissue-specific biological response to radiation damage over protracted radiotherapy treatments. Two methods (and their exten- 
sions) to compute biological doses for PIPB patients are compared: the biologically effective dose (BED) and the isoeffective dose (IED). Additionally, a comparison is made between two methods to evaluate tumour control probability (TCP), which is the clinical endpoint of interest and describes the likelihood of a treatment to be curative. These radiobiological models depend on biological parameters which are generally unknown for a given patient, so reference values are assumed (Table 6.1) unless noted otherwise.

Table 6.1: Reference radiobiological model parameters assumed in this study, generally obtained from TG-137. ${ }^{66} b$ and $d$ are from from Zaider and Minerbo. ${ }^{113}$

\begin{tabular}{lc}
\hline \hline Parameter & Reference Value \\
\hline Single-hit radiosensitivity: $\alpha$ & $0.15 \mathrm{~Gy}^{-1}$ \\
Double-hit radiosensitivity: $\beta$ & $0.05 \mathrm{~Gy}^{-2}$ \\
I-125 half life: $t_{1 / 2}$ & 59.4 days \\
I-125 decay constant: $\lambda$ & 0.01167 days \\
Tumour potential doubling time: $T_{\text {pot }}$ & 42 days \\
Effective tumour repopulation rate: $\gamma$ & 0.0165 days \\
Repair half-life: $T_{R 1 / 2}$ & 0.01125 days \\
Sub lethal repair constant: $\mu$ & 61.61 days $^{-1}$ \\
Initial number of cancer cells: $N_{0}$ & $10^{6}$ \\
Cell birth rate: $b$ & 0.066 days $^{-1}$ \\
Cell death rate: $d$ & 0.033 days $^{-1}$ \\
\hline
\end{tabular}

A well adopted ${ }^{66,114-117}$ PIPB BED formalism was presented by Dale ${ }^{67,68}$ and is a product of the physical dose and its relative effectiveness and then corrected by a repopulation factor which assumes continuous, uniform, exponential tumour growth during the treatment time. BED is often evaluated at a particular time, $T_{\text {eff }}$, which is when the rate of cell repopulation surpasses the exponentially decreasing 
rate of cell kill as the radioisotope decays:

$$
B E D\left(T_{e f f}\right)=D\left(T_{e f f}\right) R E\left(T_{e f f}\right)-\ln 2 \frac{T_{e f f}}{\alpha T_{p o t}},
$$

where,

$$
\begin{gathered}
T_{e f f}=\frac{-1}{\lambda} \ln \left(\frac{\gamma}{\alpha R_{0}}\right), \\
D\left(T_{e f f}\right)=\frac{R_{0}}{\lambda}\left(1-e^{-\lambda T_{e f f}}\right), \\
R E\left(T_{e f f}\right)=1+\frac{\beta}{\alpha} \frac{R_{0}}{(\mu-\lambda)} \frac{1}{\left(1-e^{-\lambda T_{e f f}}\right)}\left(1-e^{-2 \lambda T_{e f f}}-\frac{2 \lambda}{(\mu+\lambda)}\left(1-e^{-(\mu+\lambda) T_{e f f}}\right)\right),
\end{gathered}
$$

and $R_{0}$ is the initial dose rate (other parameters defined in Table 6.1). Implementations of BED vary across the literature. For example, Stock et al. ${ }^{114}$ did not consider the repopulation term in eq 6.1 and assumed a uniform delivery of the patient-specific $D_{90}$, where as Ling et al. ${ }^{118}$ assumed a uniform delivery of $D_{99}$.

Eq 6.3 assumes dose rate $\left(R_{0}\right)$ is constant throughout the target volume, which is untrue for PIPB dose distributions. Accounting for dose rate is important when modelling biological response since cells require time to repair damage and repopulate. In fact, with no cell proliferation and instantaneous cell repair, BED simplifies to the total physical dose. $\operatorname{Ling}^{118}$ showed that accounting for dose rate may change the biological dose by up to $70 \%$ and proposed a method to account for non-uniform dose distributions by evaluating BED separately for each isodose volume, which may be determined from the bins of a differential dose volume histogram $(\mathrm{dDVH})$. The equivalent uniform BED (EUBED) is defined as

$$
E U B E D\left(T_{e f f}\right)=\frac{-1}{\alpha} \ln \left(\sum_{i} \nu_{i} e^{-\alpha B E D_{i}}\right)
$$

where $\nu_{i}$ is the fraction of the target volume which receives the isodose, and the sum 
of all $\nu_{i}$ is 1 . EUBED has been supported by TG-13766 and implemented by several investigators. ${ }^{118-122}$

BED (and EUBED) are often used to calculate TCP using binomial (specifically, Poisson) statistics. If $n$ cells are exposed to a dose $D$, then a fraction $S(D)$ will survive. For the next $n$ cells exposed to a dose $D$, the possible range of surviving cells is anywhere between 0 and $n$, but on average there will be $n S(D)$ survivors,

$$
T C P_{P}(D)=(1-S(D))^{n} \cong e^{-n S(D)}
$$

The Poisson TCP formalism, ${ }^{66} T C P_{P}$, can be evaluated at the effective treatment time, $T_{\text {eff }}$, by assuming $N_{0}$ uniformly distributed initial cancerous cells that are subjected to a biological dose (e.g. BED),

$$
T C P_{P}\left(T_{e f f}\right)=e^{-N_{0}\left(e^{-\alpha B E D}\right)}
$$

It has been noted that the mechanistic form of $T C P_{P}$ is unrealistically steep, and Webb and Nahum ${ }^{69}$ proposed to sample $\alpha$ from a normal distribution (using $10^{5}$ samples) which effectively broadens the $T C P_{P}$ curve into what will be referred to herein as the broad TCP $\left(T C P_{B}\right)$,

$$
T C P_{B}\left(T_{e f f}\right)=\frac{1}{10^{5}} \sum_{k=1}^{10^{5}} e^{-N_{0}\left(e^{-\alpha_{k} B E D}\right)} .
$$

The uncertainty on $\alpha$, which corresponds to the standard deviation of $\alpha$ used for sampling, is not well known. The $\alpha$ broadening factor has been suggested to be 0.04 $\mathrm{Gy}^{-1}$ (Ref. ${ }^{123}$ ) or $0.08 \mathrm{~Gy}^{-1}$ (Ref. ${ }^{124}$ ), but we have assumed a lower value of 0.025 $\mathrm{Gy}^{-1}$ to mitigate over-flattening of the $T C P_{B}$ curve. Haworth et al. ${ }^{120}$ investigated the use of low $\alpha$ cut-offs and log-normal (rather than Gaussian) $\alpha$ distributions, neither of which are used in this work. 
A second approach to evaluate biological dose and TCP is described by Zaider et al. ${ }^{113,125}$ which utilizes the linear quadratic dose response (LQ) and considers natural cell proliferation and death rates explicitly. Cell surviving fraction may be described by the linear quadratic (LQ) function if one accounts for the effects of dose rate by introducing a term $q(t)$ in front of $\beta$. The term $\beta$ describes the yield of cell kill caused by two separate tracks of radiation, and is influenced by dose rate since a higher dose rate would increase the probability of the second track occurring before the first is repaired. The LQ surviving fraction and PIPB $q(t)$ are,

$$
\begin{gathered}
S(D)=e^{-\alpha D-\beta q(t) D^{2}} \\
q(t)=\frac{2(\lambda t)^{2}}{(\mu t)^{2}\left(1-\frac{\lambda^{2}}{\mu^{2}}\right)\left(1-e^{-\lambda t}\right)^{2}}\left[e^{-(\lambda+\mu) t}+\mu t\left(\frac{1-e^{-2 \lambda t}}{2 \lambda t}\right)-\frac{1+e^{-2 \lambda t}}{2}\right] .
\end{gathered}
$$

Zaider and Hanin ${ }^{125}$ extended this to calculate an isoeffective dose (IED), ${ }^{125}$ which is conceptually similar to BED,

$$
I E D(t)=\frac{-1}{\alpha} \ln \left(\frac{S(t) e^{(b-d) t}}{1+b S(t) e^{(b-d) t} \int_{0}^{t} \frac{d t^{\prime}}{S\left(t^{\prime}\right) e^{(b-d) t^{\prime}}}}\right) .
$$

Zaider and Hanin ${ }^{125}$ showed evaluating IED at $T_{\text {eff }}$ may underestimate TCP, and suggest IED be evaluated at infinite time, which, can be practically estimated as ten I-125 half lives $\left(\mathrm{t}=10 \mathrm{t}_{1 / 2}=594\right.$ days $)$.

Zaider and Minerbo ${ }^{113}$ presented a generalized TCP formalism which may be applied to permanent implant brachytherapy, which will be denoted, $T C P_{Z}$,

$$
T C P_{Z}(t)=\left(1-\frac{S(t) e^{(b-d) t}}{1+b S(t) e^{(b-d) t} \int_{0}^{t} \frac{d t^{\prime}}{S\left(t^{\prime}\right) e^{(b-d) t^{\prime}}}}\right)^{n}
$$


This form is very similar to eq 6.6, except $\mathrm{S}(\mathrm{D})$ is re-parametrized in terms of time and is modified by an additional factor which accounts for the rate of cell birth (b) and death (d).

\subsubsection{Coupling MBDCAs with Radiobiological Models}

Calculations of EUBED are highly sensitive to low doses in the $\mathrm{dDVH}^{69,126,127}$ which can reduce EUBED enough to yield a near zero TCP. To circumvent this, previous studies using water-based TG-43 dose distributions have first removed low doses (below 110 Gy by King et $a l^{122}$ and below $D_{99}$ by Ling et $a l^{118}$ ) from the dDVH before re-normalizing and calculating EUBED. This was justified by the observation that low doses typically exist near the periphery of the treatment volume and the subjective drawing of contours on post-implant images should not significantly affect the EUBED and TCP calculations. This assumption is no longer true with MC dose calculations in heterogeneous tissue models which may identify low doses in the prostate interior due to calcification shielding.

We evaluate EUBED (eq 6.5), $T C P_{P}$ (eq 6.7) and $T C P_{B}$ (eq 6.8) in two example patients with large calcifications using two MC dose calculation sets: TG43sim, simulating AAPM TG-43 conditions, and, MCDmm, which models tissues, calcifications and interseed effects with dose scored to the simulated tissues (Figure 6.1). For these two example patients, and then the whole 613 patient cohort, we compare

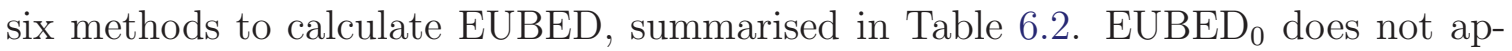
ply any low-dose cut-off, and is used to illustrate low-dose sensitivity. EUBED 1 and EUBED $_{2}$ follow the previous approaches of Ling ${ }^{118}$ and King ${ }^{122}$ and remove doses below $D_{99}$ and 110 Gy respectively. EUBED $_{3-5}$ are modified versions of EUBED $_{2}$ and take additional steps to differentiate dose contributions which may and may not 
be reasonably rejected. Since EUBED is sensitive to even a few low dose voxels, we additionally apply a global dose threshold of 72.5 Gy (half the prescription dose) for EUBED $_{3-5}$, which minimally affects these two patients as they both have a MCDmm and TG43sim $V_{50}$ of $99.7 \%$ and $100 \%$, respectively. EUBED E $_{3}$ rejects doses below 110 Gy if they are within a fixed distance from the edge of the prostate, demonstrated using 8 voxels (approximately $2.5 \mathrm{~mm}$ ). EUBED 4 rejects doses below 110 Gy except those within a distance ( 8 voxels) of any voxel assigned to calcification. EUBED $_{5}$ rejects doses below 110 Gy unless the ratio of MCDmm/TG43sim doses are sufficiently different (using a threshold of 0.6), which may identify dose perturbations due to tissue heterogeneities (Figure $6.1 \mathrm{~g}, \mathrm{~h}$ ). We do not apply EUBED 5 to TG43sim dose calculations since they would be equivalent to $\mathrm{EUBED}_{2}$. For each method to compute EUBED, $T C P_{P}$ and $T C P_{B}$ are calculated using the values in Table 6.1 and an $\alpha$ broadening factor of $0.025 \mathrm{~Gy}^{-1}$.

Table 6.2: Descriptions of the implemented PIPB radiobiological dose models.

\begin{tabular}{lll}
\hline \hline Model & Eq. & Description \\
\hline BED-NR & 6.1 & Omit the repopulation term and assume uniform $D_{90}$ dose. \\
BED- $D_{90}$ & 6.1 & Uniform $D_{90}$ dose. \\
IED- $D_{90}$ & 6.11 & Uniform $D_{90}$ dose. \\
BED- $D_{99}$ & 6.1 & Uniform $D_{99}$ dose. \\
IED- $_{90}$ & 6.11 & Uniform $D_{99}$ dose. \\
EUBED $_{0}$ & 6.5 & No low dose rejection. \\
EUBED $_{1}$ & 6.5 & Method of Ling et al. ${ }^{118}$ Reject all doses below $D_{99}$. \\
EUBED $_{2}$ & 6.5 & Method of King et al. ${ }^{122}$ Reject all doses below 110 Gy. \\
EUBED $_{3}$ & 6.5 & $\begin{array}{l}\text { Reject doses below } 110 \text { Gy only if they are near } \\
\text { the edge of the contoured target volume. } \\
\end{array}$ \\
EUBED $_{4}$ & 6.5 & $\begin{array}{l}\text { Reject all doses below } 110 \text { Gy except } \\
\text { those which are near the edge of calcification. }\end{array}$ \\
EUBED $_{5}$ & 6.5 & $\begin{array}{l}\text { Reject all doses below } 110 \text { Gy except those which the } \\
\text { ratio of MCDmm/TG43sim doses are sufficiently low. }\end{array}$ \\
\hline
\end{tabular}

For 613 PIPB patient MCDmm and TG43sim calculated dose distributions, several biological dose models are evaluated (Table 6.2). BED (eq 6.1) is eval- 

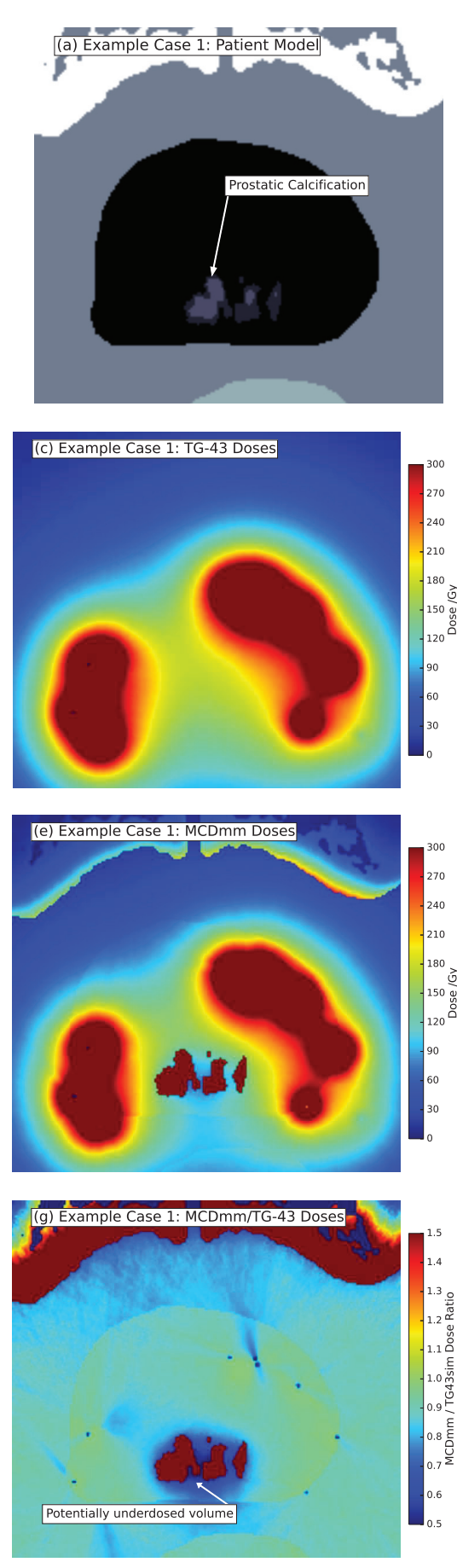
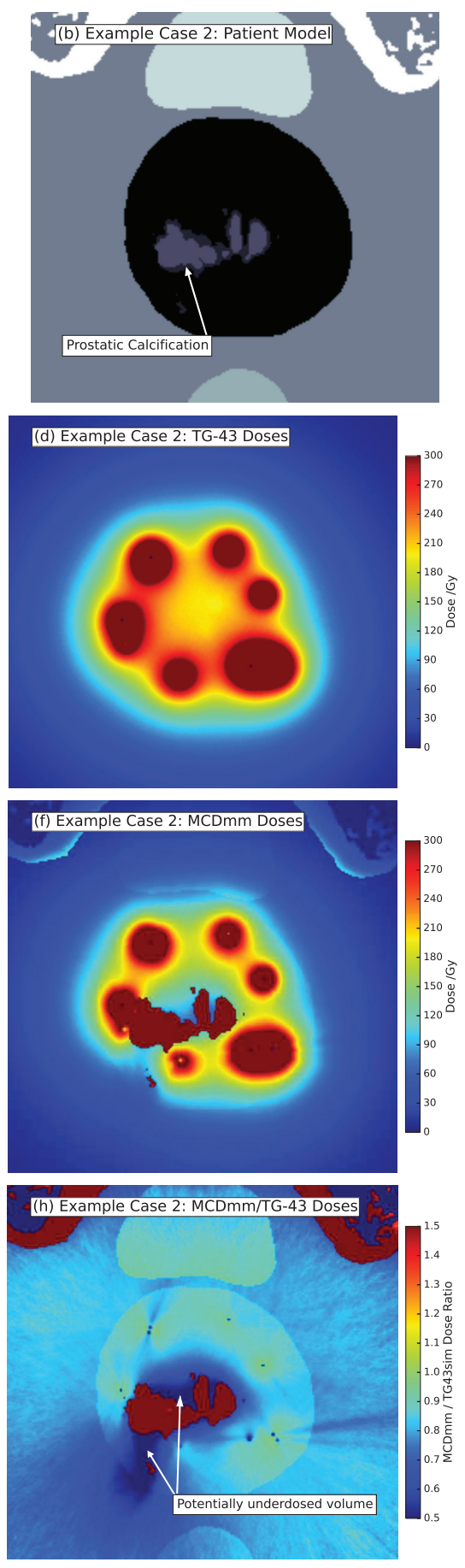

Figure 6.1: Two example patients with prostatic calcifications. (a,b) shades of grey represent different tissues assigned in the MCDmm virtual patient model, showing the location of the prostate and calcifications. (c,d) TG43sim dose distribution, $(\mathrm{e}, \mathrm{f}) \mathbf{M C D m m}$ dose distribution and $(\mathrm{g}, \mathrm{h}) \mathbf{M C D m m} / \mathbf{T G}$ 43sim dose ratio. For example patient 1, MCDmm (TG43sim) $D_{90}$ is 128.3 (151.8) Gy. For example patient 2, MCDmm (TG43sim) $D_{90}$ is 110.4 (137.4) Gy. 
uated at $T_{\text {eff }}$ under various conditions: BED-NR assumes no repopulation (omit last term of eq 6.1) and a uniform delivery of $D_{90}, \mathrm{BED}-D_{90}$ assumes a uniform delivery of $D_{90}$ and BED- $D_{99}$ assumes a uniform delivery of $D_{99}$. Similarly, IED (eq 6.11) is evaluated at 594 days $\left(10 \mathrm{t}_{1 / 2}\right)$, where IED- $D_{90}$ assumes a uniform delivery of $D_{90}$, and IED- $D_{99}$ assumes a uniform delivery of $D_{99}$. EUBED (eq 6.5) is calculated using the six aforementioned approaches. When applying these EUBED methods to the large patient cohort, it is likely that some patients have doses below 72.5 Gy and are affected by our assumed cut-off. However, this should be rare as the cohort average $V_{50}$ is $99.3 \%$ for MCDmm doses and $99.5 \%$ for TG43sim doses. For each biological dose, an associated TCP is calculated; BED and EUBED are used to derive $T C P_{P}$ (eq 6.7) and with $T C P_{Z}$ (eq 6.12) corresponding to IED.

\subsection{Results}

\subsubsection{Example patients with calcifications}

Figure 6.2 represents the different low dose voxels rejected in two example patients with large calcifications by the alternate EUBED models described in Table 6.2. With water-based dose calculations, $\mathrm{EUBED}_{1}$ and $\mathrm{EUBED}_{2}$ are assumed to reject low doses only near the periphery of the target contour, yet rejected doses can be several millimeters towards the target volume interior (Figure 6.2 b,d). Applying EUBED $_{1}$ and $\mathrm{EUBED}_{2}$ to $\mathbf{M C D m m}$ dose calculations can lead to the unwanted rejection of low doses which are caused by calcification shielding (Figure $6.2 \mathrm{a}, \mathrm{c}, \mathrm{j}, \mathrm{l}$ ), supporting the consideration of $\mathrm{EUBED}_{3-5} \cdot \mathrm{EUBED}_{3}$, which is designed to only reject low doses near the subjective target volume boundary may also reject low doses which are as a result of calcification shielding if the calcification is near the 
edge of the target (Figure 6.2 e,n). $\mathrm{EUBED}_{4}$, which preserves low doses near voxels corresponding to calcification in the MCDmm virtual patient model, identifies very few voxels in TG43sim models, but those due to calcification shielding in MCDmm models (Figure 6.2 g,h,p,q). EUBED 5 appears to reasonably differentiate between low dose voxels near the target periphery and those due to calcification shielding, however less voxels are preserved surrounding the calcification compared to $\mathrm{EUBED}_{3}$ and $\mathrm{EUBED}_{4}$ in one patient (Figure 6.2 e,g,i).

When evaluating EUBED for these two example cases, Table 6.3, it is clear that $\mathrm{EUBED}_{0}$ is sensitive to low doses and is considerably lower than $\mathrm{EUBED}_{1-5}$ (by $23 \%$ at minimum). $\mathrm{EUBED}_{1}$ is lower than $\mathrm{EUBED}_{2}$ in both patients for $\mathbf{M C D m m}$ and TG43sim since the $D_{99}$ dose threshold is lower than 110 Gy (MCDmm $D_{99}$ is 85.2 and 84.5 Gy, and TG43sim $D_{99}$ is 100.1 and 98.4 Gy for patients 1 and 2 respectively). MCDmm EUBED $_{3-5}$ are similar to each other, but are lower than $\mathrm{EUBED}_{2}$ since they each also include some voxels below 110 Gy which further lowers EUBED. The calculated EUBEDs are between 4.1 and 24.5 Gy lower with MCDmm compared to TG43sim, which causes a large difference in $\mathrm{TCP}_{P}$ (8.0 to $85.8 \%$ lower with MCDmm). Even when assuming a broader tumour response, $\mathrm{TCP}_{B}$ is 7.5 to 49.1\% lower with MCDmm compared to TG43sim.

\subsubsection{Large Patient Cohort}

The results of applying the various biological dose models to the 613 patient cohort MCDmm and TG43sim dose calculations are provided in Figure 6.3, with the means and standard deviations in Table 6.4. The considerable variation in doses between patients is responsible for the large variation in biological doses and TCPs for any given model (MCDmm $D_{90}$ of 82.7-201.4 Gy for 95\% of patients and MCDmm 

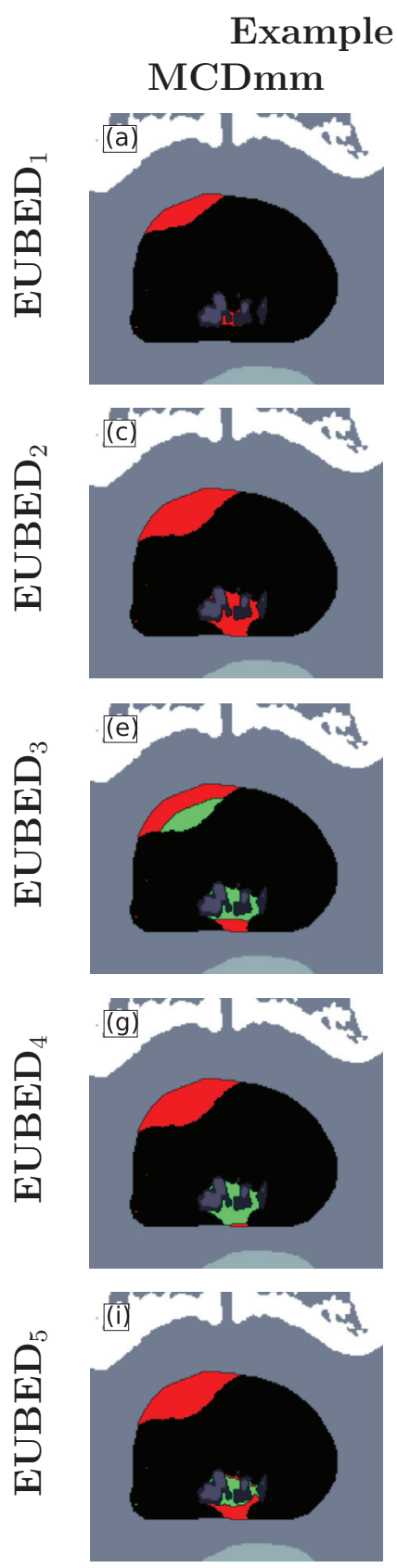

Example Patient 2
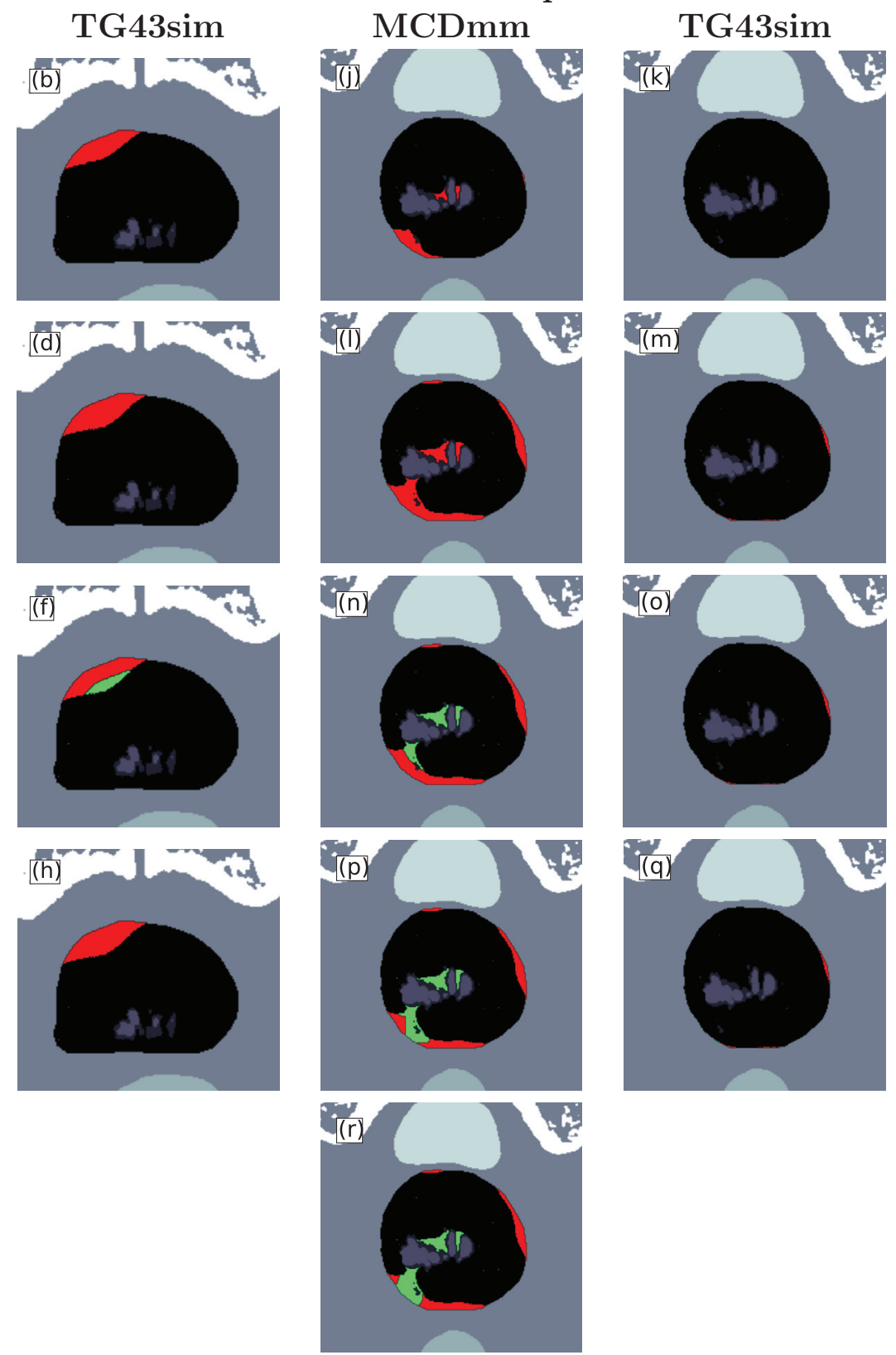

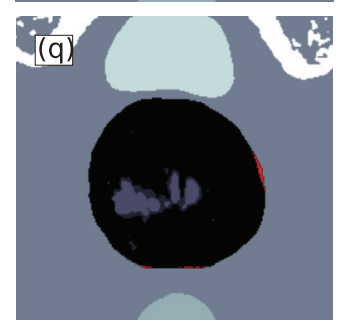

TG43sim
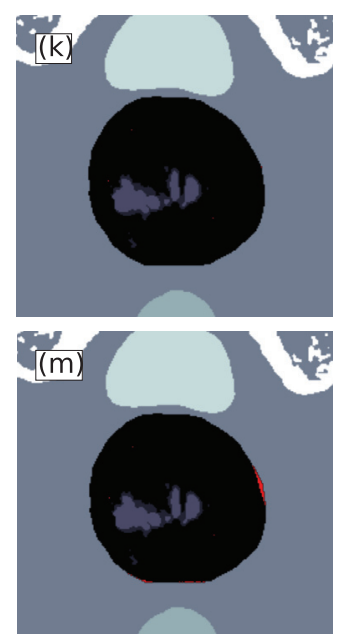

Figure 6.2: Two example patients with calcifications (a-i and j-r) illustrating the low dose voxels identified by EUBED $_{1-5}$ (Table 6.2) using MCDmm and TG43sim dose calculations (corresponding to Figure 6.1). Black is the target volume, and shades of grey are different tissues assigned in the MCDmm virtual patient model. $(\mathrm{a}, \mathrm{b}, \mathrm{j}, \mathrm{k})$ EUBED $_{1}$ : rejected doses below $D_{99}$ in red. (c,d,l,m) EUBED E $_{2}$ rejected doses below

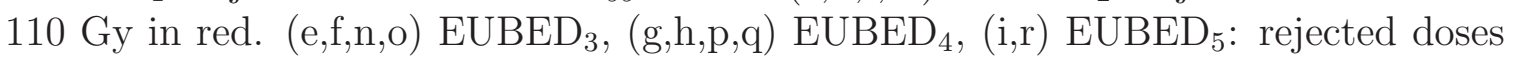
below 110 Gy in red, and those identified as low doses to preserve in green (greens are rejected by $\mathrm{EUBED}_{2}$ ). Calculated EUBEDs and TCPs for these example patients are in Table 6.3. 
Table 6.3: Various approaches to calculated EUBED (eq 6.5), $T C P_{P}$ (eq 6.7) and $T C P_{B}$ (eq 6.8) using MCDmm dose calculations for two example patients (Ex. Pt.) with calcifications (Figure 6.2) that result in low dose regions within the target volume.

\begin{tabular}{|c|c|c|c|c|c|c|c|}
\hline \multirow[b]{2}{*}{$\begin{array}{l}\text { Ex. } \\
\text { Pt. }\end{array}$} & \multirow[b]{2}{*}{ Model } & \multicolumn{3}{|c|}{ MCDmm } & \multicolumn{3}{|c|}{ TG43sim } \\
\hline & & $\begin{array}{c}\text { EUBED } \\
{[\mathrm{Gy}]}\end{array}$ & $\begin{array}{c}T C P_{P} \\
{[\%]}\end{array}$ & $\begin{array}{c}T C P_{B} \\
{[\%]}\end{array}$ & $\begin{array}{c}\text { EUBED } \\
{[\mathrm{Gy}]}\end{array}$ & $\begin{array}{c}T C P_{P} \\
{[\%]}\end{array}$ & $\begin{array}{c}T C P_{B} \\
{[\%]}\end{array}$ \\
\hline \multirow[t]{6}{*}{1} & $\overline{\text { EUBED }_{0}}$ & 69.9 & 0.0 & 2.9 & 85.3 & 6.1 & 25.9 \\
\hline & $\mathrm{EUBED}_{1}$ & 86.3 & 9.0 & 28.5 & 101.7 & 78.8 & 62.6 \\
\hline & $\mathrm{EUBED}_{2}$ & 103.8 & 84.1 & 66.5 & 108.7 & 92.1 & 74.4 \\
\hline & $\mathrm{EUBED}_{3}$ & 89.6 & 23.2 & 36.2 & 109.2 & 92.6 & 74.8 \\
\hline & $\mathrm{EUBED}_{4}$ & 89.6 & 23.3 & 36.8 & 108.7 & 92.0 & 74.6 \\
\hline & $\mathrm{EUBED}_{5}$ & 90.2 & 26.3 & 37.0 & - & - & - \\
\hline \multirow[t]{6}{*}{2} & $\mathrm{EUBED}_{0}$ & 55.7 & 0 & 0 & 87.1 & 12.0 & 30.3 \\
\hline & $\mathrm{EUBED}_{1}$ & 81.9 & 0.9 & 19.0 & 98.4 & 68.0 & 56.9 \\
\hline & $\mathrm{EUBED}_{2}$ & 101.0 & 76.9 & 61.0 & 105.1 & 86.8 & 68.5 \\
\hline & $\mathrm{EUBED}_{3}$ & 85.7 & 7.8 & 27.4 & 110.2 & 93.6 & 76.5 \\
\hline & $\mathrm{EUBED}_{4}$ & 83.5 & 2.7 & 22.2 & 103.7 & 84.0 & 66.4 \\
\hline & $\mathrm{EUBED}_{5}$ & 83.1 & 2.1 & 21.5 & - & - & - \\
\hline
\end{tabular}

$D_{99}$ of 42.1-143.4 Gy for 95\% of patients). Calculations of BED and IED assuming a uniform delivery of the 145 Gy prescription dose yield 111.1 and 103.4 Gy respectively, and TCPs of 94.4 and $83.2 \%$ respectively (not shown in Figure 6.3 or Table 6.4). BED-NR is considerably higher than the other biological doses since it does not account for cell repopulation, which underestimates the ability for a tumour to regenerate over the treatment time. Assuming a uniform delivery of $D_{90}$ rather than $D_{99}$ returns a higher $\mathrm{MCDmm}$ BED by $42 \%$ and IED by $41 \%$, since the cohort average $D_{90}$ is larger than the average $D_{99}$ by $34 \%$. Average $\mathrm{EUBED}_{0}$ is lower than EUBED $_{1-5}$ for both MCDmm and TG43sim dose distributions, but higher than BED and IED calculated with $D_{99}$. EUBED 2 values have a smaller range than those for $\mathrm{EUBED}_{1,3,4,5}$ since no doses below 110 Gy are ever considered, regardless of the implant quality or the spatial distribution of doses.

Average physical dose metrics evaluated with MCDmm are a few percent 
lower than with TG43sim (average $D_{90}$ and $V_{100}$ are lower by $5.9 \%$ and $2.6 \%$ with MCDmm). This results in a similar difference in biological doses (Table 6.4), with average MCDmm BED-NR lower by $5.9 \%, \operatorname{BED}\left(D_{90}\right)$ by $7.4 \%$ and $\operatorname{EUBED}_{2}$ by 1.9\%. These differences in average biological dose cause differences in TCP estimates, with smaller biological doses leading to larger TCP differences; average MCDmm BED-NR $T C P_{P}$ is lower by $1.7 \%, \operatorname{BED}\left(D_{90}\right) T C P_{P}$ by $13.4 \%$ and $\mathrm{EUBED}_{2} T C P_{P}$ by $1.9 \%$.

Table 6.4: Mean biological doses, in Gy, and TCPs, in \%, calculated for 613 PIPB patients using MCDmm and TG43sim dose calculations. $\bar{x}$ corresponds to the mean, $\sigma$ is the standard deviation and $\Delta$ is the percent difference between the means.

\begin{tabular}{|c|c|c|c|}
\hline $\begin{array}{l}\text { RadBio. } \\
\text { Model }\end{array}$ & $\begin{array}{c}\text { MCDmm } \\
\bar{x} \pm \sigma\end{array}$ & $\begin{array}{c}\text { TG43sim } \\
\bar{x} \pm \sigma\end{array}$ & $\Delta \%$ \\
\hline$\overline{B E D-N R}$ & $144.6 \pm 29.6$ & $153.3 \pm 31.0$ & -5.9 \\
\hline BED- $D_{90}$ & $109.8 \pm 27.7$ & $117.9 \pm 29.1$ & -7.4 \\
\hline IED- $D_{90}$ & $102.3 \pm 25.8$ & $109.9 \pm 27.2$ & -7.4 \\
\hline BED- $D_{99}$ & $63.3 \pm 23.7$ & $69.5 \pm 24.9$ & -9.8 \\
\hline IED- $D_{99}$ & $60.1 \pm 21.1$ & $65.6 \pm 22.4$ & -9.2 \\
\hline $\mathrm{EUBED}_{0}$ & $75.0 \pm 15.9$ & $86.5 \pm 23.0$ & -15.4 \\
\hline EUBED $_{1}$ & $94.9 \pm 24.7$ & $101.4 \pm 25.9$ & -6.9 \\
\hline $\mathrm{EUBED}_{2}$ & $109.3 \pm 8.2$ & $111.3 \pm 9.9$ & -1.9 \\
\hline $\mathrm{EUBED}_{3}$ & $88.7 \pm 18.0$ & $101.5 \pm 20.8$ & -14.4 \\
\hline $\mathrm{EUBED}_{4}$ & $106.7 \pm 12.6$ & $109.8 \pm 12.3$ & -2.9 \\
\hline EUBED $_{5}$ & $92.8 \pm 14.6$ & - & \\
\hline TCP $_{P}:$ BED-NR & $95.4 \pm 16.8$ & $97.0 \pm 13.3$ & -1.7 \\
\hline $\mathrm{TCP}_{P}:$ BED- $D_{90}$ & $68.6 \pm 38.5$ & $77.8 \pm 34.4$ & -13.4 \\
\hline TCP $_{Z}:$ IED- $D_{90}$ & $58.0 \pm 40$ & $69.0 \pm 38.2$ & -19.0 \\
\hline $\mathrm{TCP}_{P}:$ BED- $D_{99}$ & $10.3 \pm 26.5$ & $14.7 \pm 31.3$ & -43.1 \\
\hline $\mathrm{TCP}_{Z}:$ IED- $D_{99}$ & $7.0 \pm 22.0$ & $10.5 \pm 26.7$ & -50.2 \\
\hline $\mathrm{TCP}_{P}: \mathrm{EUBED}_{0}$ & $10.4 \pm 25.1$ & $31.3 \pm 39.0$ & -200.2 \\
\hline $\mathrm{TCP}_{P}: \mathrm{EUBED}_{1}$ & $45.9 \pm 41.4$ & $56.7 \pm 41.6$ & -23.7 \\
\hline $\mathrm{TCP}_{P}: \mathrm{EUBED}_{2}$ & $89.8 \pm 6.2$ & $91.4 \pm 5.7$ & -1.9 \\
\hline $\mathrm{TCP}_{P}: \mathrm{EUBED}_{3}$ & $34.4 \pm 39.3$ & $62.3 \pm 40.4$ & -81.3 \\
\hline $\mathrm{TCP}_{P}: \mathrm{EUBED}_{4}$ & $82.7 \pm 23.5$ & $87.2 \pm 18.8$ & -5.4 \\
\hline $\mathrm{TCP}_{P}: \mathrm{EUBED}_{5}$ & $43.0 \pm 41.4$ & - & - \\
\hline
\end{tabular}



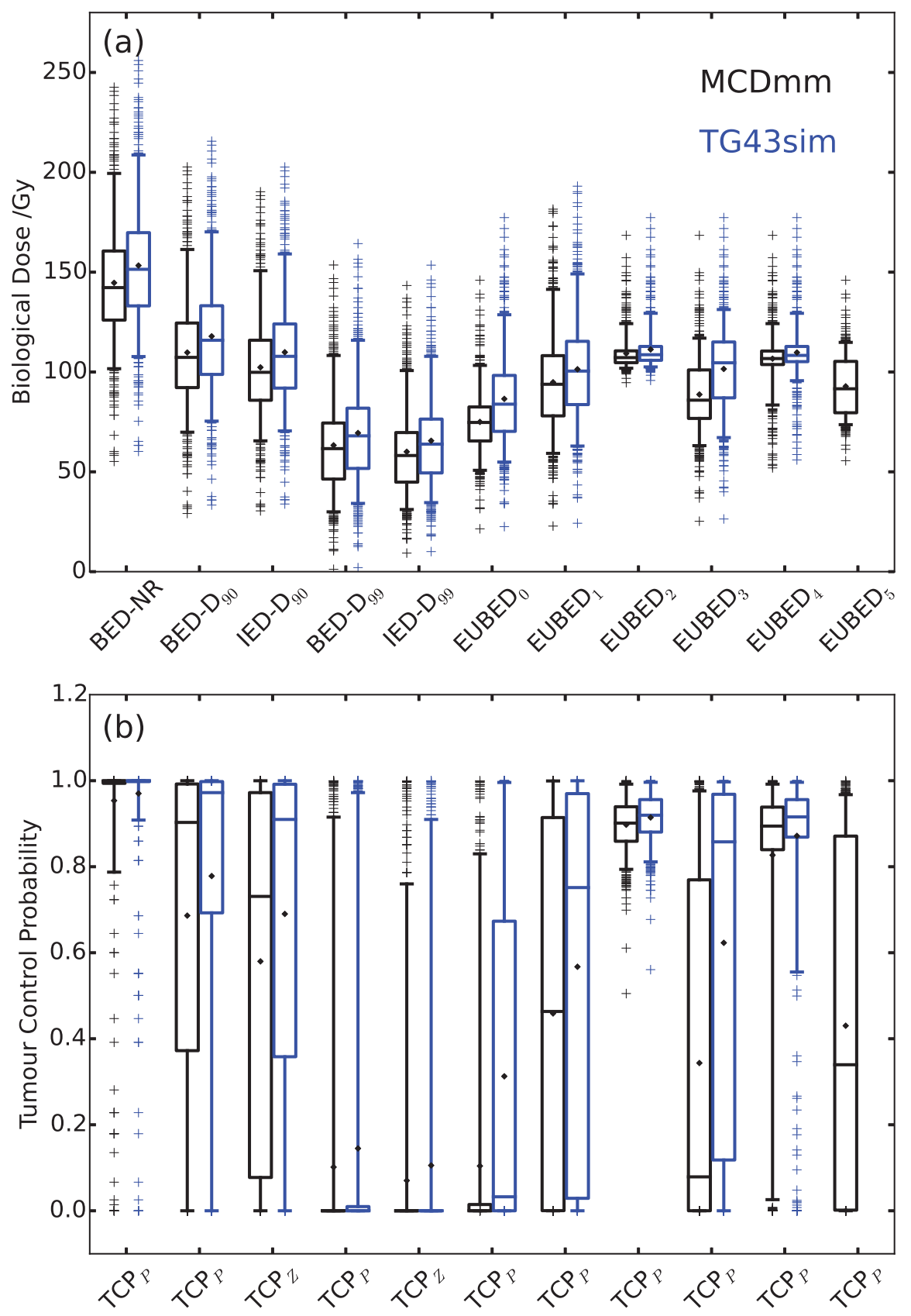

Figure 6.3: Biological doses and TCPs evaluated for 613 permanent implant prostate brachytherapy retrospective Dmm (black) and TG43sim (blue) Monte Carlo dose calculations. Box plots represent median and 50th percentile range, whiskers extend to 95 th percentile, crosses are outliers and and dots represent distribution mean. The TCPs in the lower panel correspond to the biological doses along the aligned $\mathrm{x}$-axis in the top panel. 
Although the average dose metrics calculated with MCDmm are within a few percent of TG43sim, the MCDmm voxel-by-voxel dose distributions can be quite different (Figure $6.1 \mathrm{~g}, \mathrm{~h}$ ). Biological dose models which do not account for dose heterogeneity, such as $\operatorname{BED}\left(D_{90}\right)$, and do not consider lower dose volumes, such as EUBED $_{2}$, are well correlated between MCDmm and TG43sim. However, the new EUBED models presented herein, such as EUBED 5 , which is affected by any low doses in MCDmm due to tissue heterogeneity, can lead to large differences between MCDmm and TG43sim biological doses (Figure 6.4a).

There is a considerable range of biological doses evaluated over the large patient cohort depending on how complicated the model (Figure 6.3). Assuming a uniform dose of $D_{90}$, as we have done for $\operatorname{BED}-\mathrm{NR}, \operatorname{BED}\left(D_{90}\right)$ and $\operatorname{IED}\left(D_{90}\right)$, results in biological doses which differ, but are directly proportional to each other. However, biological dose models which assume uniform delivery of $D_{99}$ or consider dose heterogeneity with EUBED, are not proportional to the uniform $D_{90}$ models (Figure 6.4b). 

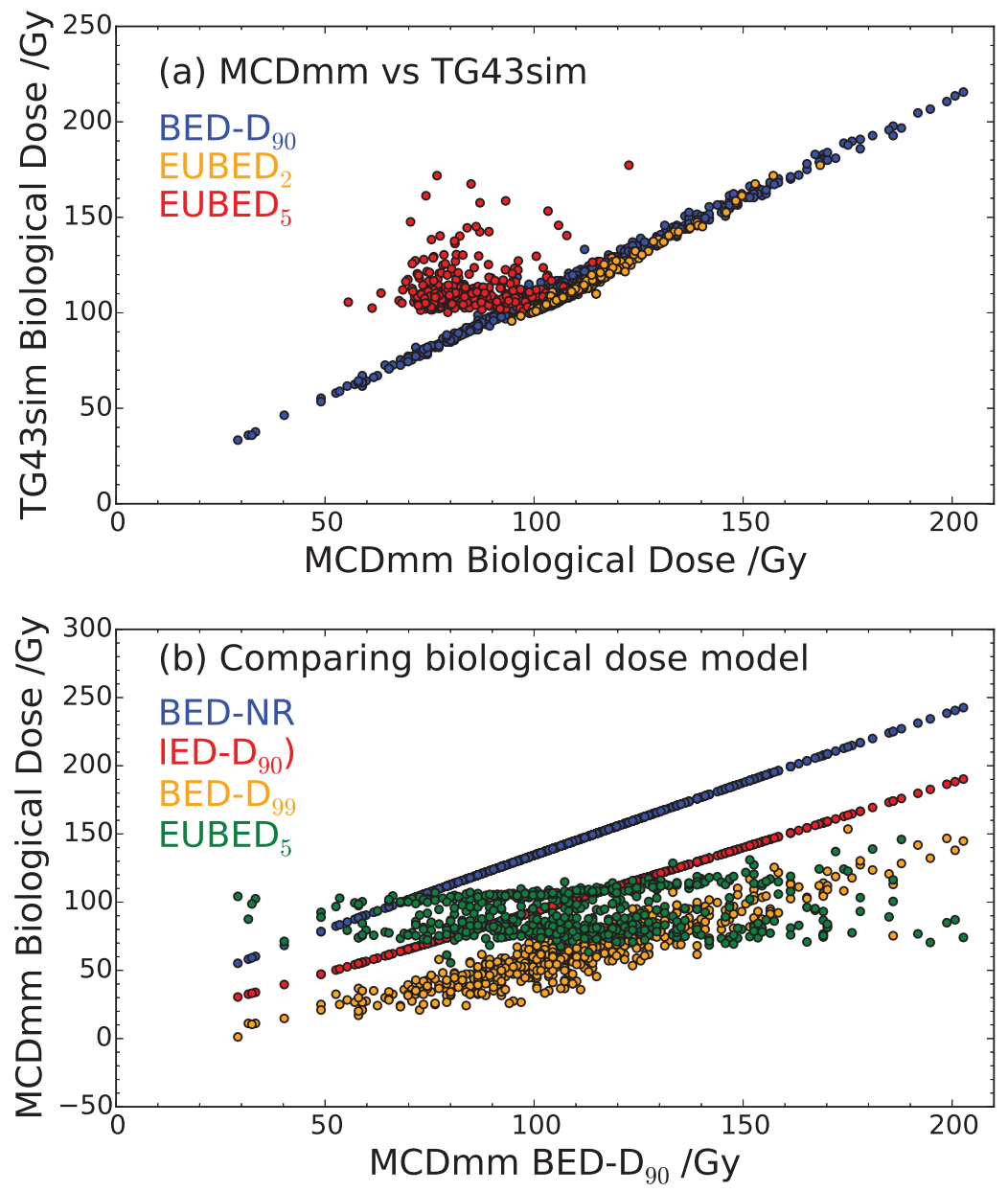

Figure 6.4: (a) Relationship between the different biological doses of 613 PIPB patients using MCDmm dose calculations. (b) Relationship between MCDmm and TG43sim dose calculations applied to different radiobiological models. For TG43sim, EUBED 5 is the same as EUBED 2 . 


\subsection{Discussion}

\subsubsection{Model Based Dose Calculations compared with TG-43}

This study is the first to explore challenges related to coupling model based PIPB dose calculations with existing radiobiological models. We have shown that there are new issues to consider when dose calculations account for prostatic calcifications, and we should seek to avoid implementing radiobiological models which cannot account for under-dosed volumes due to calcification shielding. Small differences between MCDmm and TG43sim calculated biological doses can lead to large differences in TCP estimates (Figure 6.3).

By studying two example patients with large calcifications we have illustrated that calcification shielding can exist throughout the target volume (Figure 6.1 e-h), existing EUBED paradigms $\left(\operatorname{EUBED}_{1,2}\right)$ will fail to identify these regions (Figure $6.2 \mathrm{a}-\mathrm{d}$ and $\mathrm{j}-\mathrm{m})$, and inclusion of these low dose volumes can change EUBED calculations by approximately 15-20\% leading to reductions in TCP of $60-70 \%$ (Table 6.3). The local dose differences observed between MCDmm and TG43sim should be reflected in calculations of biological dose, which are only realised with more complicated models that consider dose perturbations from tissue heterogeneities, such as EUBED $_{3,4,5}$ (Figure 6.4).

The Monte Carlo dose calculations presented herein are $D_{m, m}$, meaning simulated particles are transported through, and dose is scored in, virtual tissues. An alternate $\mathrm{MC}$ dose scoring method is $D_{w, m}$, which is similar, except dose is scored to water. Both types of dose calculations were performed for the 613 patients, with very similar physical DVH dose metrics (average $D_{90}$ and $V_{100}$ were lower by $0.7 \%$ and $0.3 \%$ with $D_{m, m}$ compared to $\left.D_{w, m}\right)$. When applying $D_{w, m}$ doses to the radiobiological 
models (results not shown), biological doses were very similar to $D_{m, m}$, with average $\operatorname{BED}\left(D_{90}\right), \mathrm{EUBED}_{2}$ and $\mathrm{EUBED}_{5}$ lower by $0.9 \%, 0.2 \%$ and $0 \%$ respectively with $D_{m, m}$. Thus, the difference between PIPB $D_{m, m}$ and $D_{w, m}$ dose calculations applied to radiobiological models is negligible compared to the other sources of variability between radiobiological models.

\subsubsection{Choice of radiobiological model}

We observe small differences between BED and IED and between $T C P_{P}$ and $T C P_{Z}$ compared to the differences due to neglecting cell repopulation or considering dose heterogeneity (Figure 6.3 and Table 6.4). IED was calculated at 594 days, which is ten ${ }^{125} \mathrm{I}$ half lives used to approximate infinite time. Additionally, we calculated IED at $\mathrm{T}_{\text {eff }}=234.21$ days to more directly compare to BED (results not shown) which reduced the average IED by less than $1 \%$. This suggests that IED is not overly sensitive to the chosen evaluation time, when taken in context of the other sources of variability between the calculated radiobiological quantities.

Although EUBED has the advantage of accounting for dose heterogeneity, it is limited by a sensitivity to the value chosen for dose cut-off (Figure 6.5). We extended the existing EUBED model to differentiate low doses which may be most important to consider in radiobiological models, presenting two methods which rely on the spatial distribution of doses $\left(\mathrm{EUBED}_{3}, \mathrm{EUBED}_{4}\right)$ and another method $\left(\right.$ EUBED $\left._{5}\right)$ which uses both fully modelled tissue and water-based dose calculations. Indeed, EUBED ${ }_{3,4,5}$ were quite different than $\mathrm{EUBED}_{2}$ in select highly calcified patients (Table 6.3). However, when applied across the 613 patient cohort, where approximately $90 \%$ of patients had no visible calcifications on post-implant CT images, their differences from $\mathrm{EUBED}_{2}$ are of the same order as differences between other 
similar radiobiological models (e.g. BED- $D_{90}$ vs BED- $D_{99}$ ) (Figure 6.3, Table 6.4).

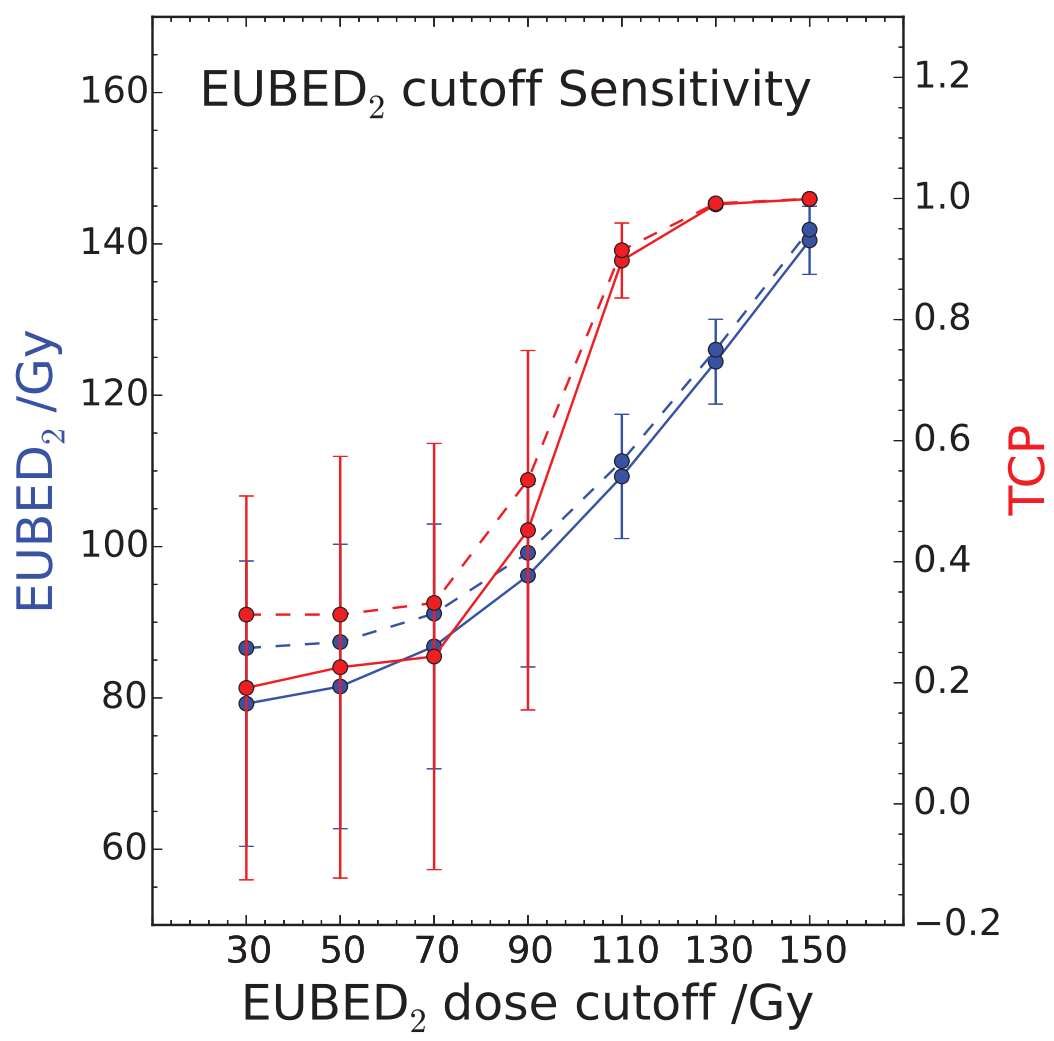

Figure 6.5: Sensitivity of MCDmm (solid line) and TG43sim (dashed line) mean EUBED $_{2}$ (blue) and mean $\mathrm{TCP}_{P}$ (red) as a function of the assumed low dose cutoff of EUBED ${ }_{2}$. Means are calculated from 613 patients, and error bars (standard deviations) are displayed only for MCDmm.

It is important to recognise that the TCPs which have been calculated herein (ranging between 0 and 100\% in Figure 6.3b) do not accurately reflect patient outcomes. The outcomes of this cohort of PIPB patients has not yet been studied, however, an earlier investigation of 157 PIPB patients from the same institution reported an $87.1 \%$ 5-year biochemical failure free survival. ${ }^{128}$ Larger outcomes studies from other institutions ${ }^{6-10}$ report 5 to 10 year biochemical failure free survival rates for similar low to intermediate risk PIPB patients generally in the range of 85-95\%. 
Indeed, investigators ${ }^{114-116}$ have correlated low BED values to poor clinical outcomes, but modelling approaches adopted for those investigations are most similar to BEDNR using TG43sim which is highly proportional to $D_{90}$ and the highest estimate of biological dose which yields a TCP of at least $90 \%$ for $95 \%$ of patients.

\subsubsection{Sensitivity to assumed biological parameters}

In this work we have implemented radiobiological models by assuming a particular set of parameter values, but these parameters can vary across patient cohorts and these models are sensitive to these assumed values. We adopt an $\alpha$ of $0.15 \mathrm{~Gy}^{-1}$, based on TG-137 recommendations and the work of Wang et al. ${ }^{123}$ but acknowledge $\alpha$ may at least be between 0.09 and $0.35 \mathrm{~Gy}^{-1} \cdot{ }^{66}$ Biological dose and TCP are highly dependent on $\alpha$, and it is unique in that increasing $\alpha$ (with a fixed $\beta$ ) can decrease EUBED and increase TCP (Figure 6.6a). Cell repopulation is important to consider since neglecting it can cause an overestimation of biological dose and TCP (BED-NR vs other biological dose models in Figure 6.3a). We have assumed a $\mathrm{T}_{\text {pot }}$ of 42 days based on TG-137 recommendations, but $\mathrm{T}_{\text {pot }}$ may at least range from 10 to 60 days. ${ }^{129}$ Calculations of EUBED and TCP rapidly decrease (Figure 6.6b) as the assumed rate of cell repopulation increases $\left(\mathrm{T}_{\text {pot }}\right.$ decreases). Figure 6.7 shows that TCP is quickly reduced when the assumed number of cells increases. Additionally, we modified eq 6.7 by replacing $\mathrm{N}_{0}$ by the product of the each patient's prostate volume and an assumed cancer cell density, but the distribution of evaluated TCPs was largely unaffected. We estimated a tumour cell density of $2.77 \times 10^{4}$ cells $/ \mathrm{cm}^{3}$ based on the nominal $\mathrm{N}_{0}$ of $10^{6}$ cells and our cohort average prostate volume of $36.04 \mathrm{~cm}^{3}$. 

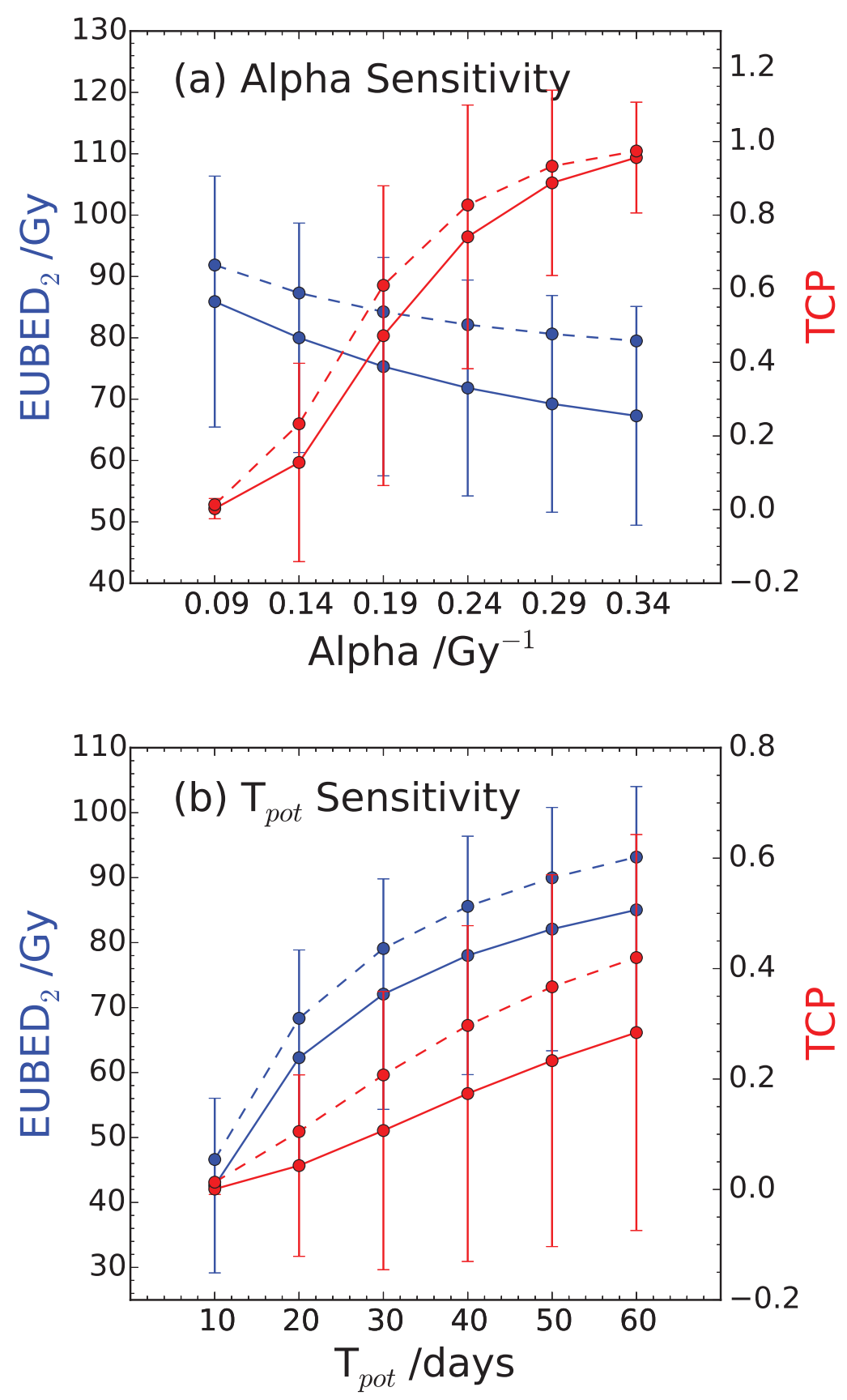

Figure 6.6: Sensitivity of MCDmm (solid line) and TG43sim (dashed line) mean EUBED $_{2}$ (blue) and mean $\mathrm{TCP}_{P}$ (red) as a function of (a) alpha and (b) $T_{p o t}$. Mean are calculated from 613 patients, and error bars (standard deviations) are displayed only for MCDmm. 


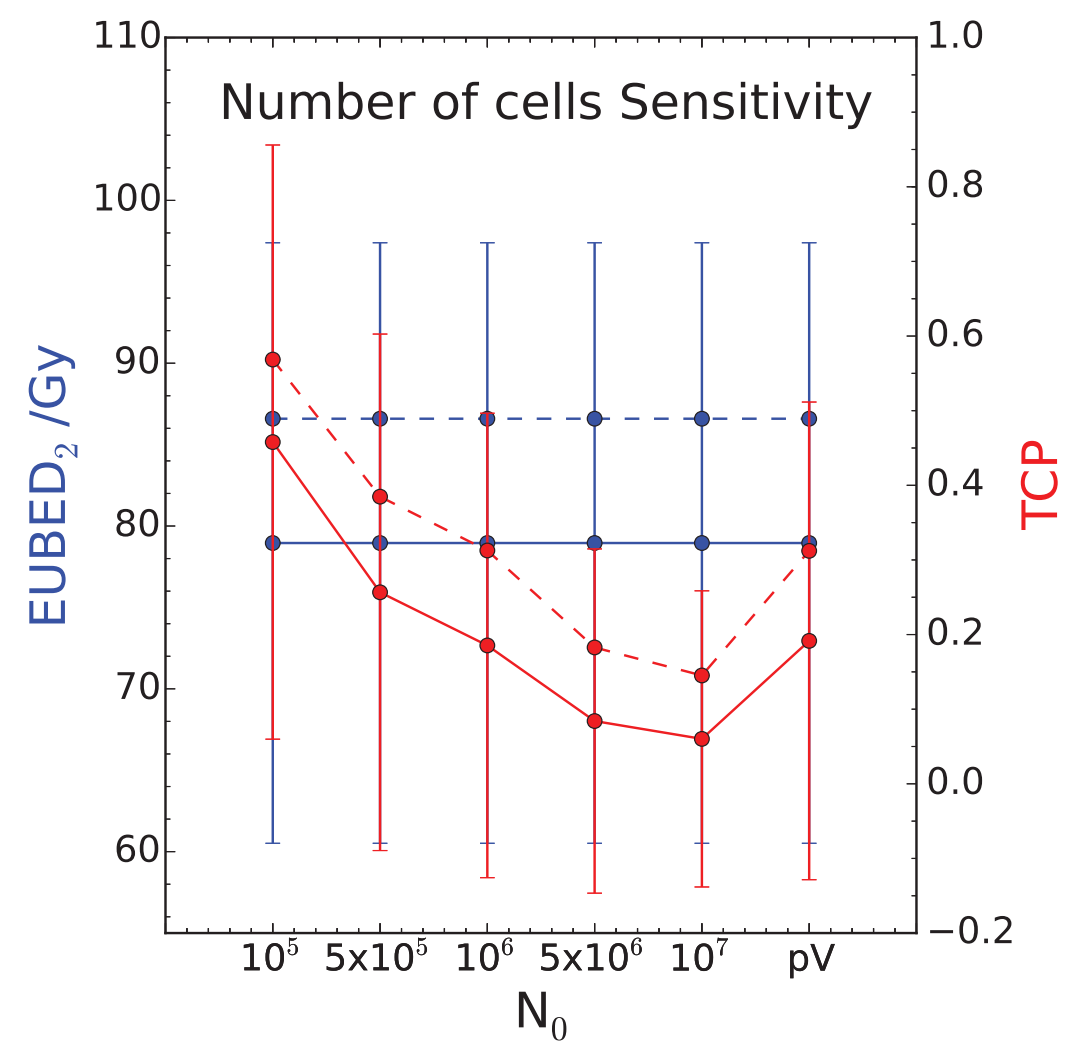

Figure 6.7: Sensitivity of MCDmm (solid line) and TG43sim (dashed line) mean EUBED $_{2}$ (blue) and mean $\mathrm{TCP}_{P}$ (red) as a function of the assumed number of cancer cells, N. Mean are calculated from 613 patients, and error bars (standard deviations) are displayed only for MCDmm.

\subsubsection{Radiobiological modelling advancements}

Although the mechanistic TCP models presented herein are flexible through their large parameter-space, many of the calculated TCPs using the TG-137 recommended parameter values are unrealistically low (Figure 6.3, Table 6.4). We have illustrated in Table 6.3 that using a broader $\mathrm{TCP}_{B}$ may increase some of the lower calculated TCPs, but it requires an additional unknown biological parameter $\left(\sigma_{\alpha}\right)$ and reduces the higher calculated TCPs. Ideally, one would adopt a phenomenological TCP model, 
but this would be a significant challenge to develop with reasonable statistical uncertainty because of the limited number of failed treatments and the overall uncertainty in dose calculations.

The Monte Carlo PIPB dose distributions studied herein may be substantially more accurate than the clinical TG-43 calculations, especially in patients with calcifications, but they are also limited by their assumption that the patient geometry is static and defined by 30-day post-implant CT images. In reality, the majority of dose delivery is within the first weeks after treatment when significant swelling and edema might considerably change the patient geometry with time. ${ }^{106-108}$ The sensitivity of the radiobiological functions to the spatial distribution of doses within the treatment volume supports the need for research towards dynamic 4D model based dose calculations for brachytherapy.

All of the radiobiological models studied herein are limited by their assumption that tumour cells are uniformly distributed throughout the entire prostate volume. Several publications ${ }^{130-132}$ using histology data have identified that subvolumes of the prostate have different probabilities of finding tumour cells. The work of Haworth et al. ${ }^{119,120}$ has presented an advanced radiobiological modelling approach which incorporates this data to account for tumour cell heterogeneity. However, this relies on population based tumour distributions and still cannot properly describe the tumour heterogeniety for any given patient. Fortunately, many institutions are now moving towards focal therapies and acquiring multiparametric MRI (mpMRI) ${ }^{133,134}$ to identify patient-specific prostate subvolumes which contain cancerous cells. A significant contribution towards the development of PIPB radiobiological modelling will involve the development of mpMRI guided heterogeneous tumour burden biological dose and TCP models. However, the models presented herein which consider heterogeneous dose distributions and aim to identify critical low dose subvolumes may 
be useful tools for retrospective radiobiology studies using patient data that lacks differentiated tumour subregions.

\subsection{Conclusions}

Several radiobiological modelling approaches have been described in the literature and implemented by researchers. Considerable differences exist in the estimated biological doses and tumour control probabilities based on which radiobiological model is assumed. Since ${ }^{125}$ I permanent implant prostate brachytherapy dose distributions are highly heterogeneous and dose is delivered over several weeks, we suggest that the most appropriate models should consider cell repopulation and dose heterogeneity. However, existing models which satisfy this criteria, such as EUBED, are limited by a large sensitivity to small low-dose volumes. Existing approximations which neglect these low doses may no longer be justified when these models are coupled with MC dose calculations which can identify clinically relevant low-dose volumes resulting from calcification shielding. Our proposed extensions to EUBED require the consideration of the spatial distribution of doses, which is more complicated than traditional EUBED evaluation based only on the DVH. Despite this added complexity, we observed that efforts to differentiate and consider low doses resulting from tissue heterogeneities can have substantial effects on biological dose and TCP estimates, suggesting it is a worthwhile endeavour. We have shown that the moderate difference between PIPB water-based TG-43 and full tissue Monte Carlo physical dose calculations can translate to large differences in TCP estimates, especially for lower estimates of biological dose. However, the differences between radiobiological model outcomes between water-based and full tissue Monte Carlo dose calculations is shadowed by the dependence of these radiobiological models on which model is 
implemented and choice of parameter values. The considerable sensitivity of these radiobiological models to assumed biological parameters continues to be a concern, especially since existing recommendations are to implement population based values which cannot accurately describe the biological response of any particular individual patient. Nevertheless, these radiobiological models may continue to be a valuable tool to qualitatively compare the biological effects of different PIPB dose distributions. 


\section{Chapter 7}

\section{Summary and Outlook}

This Chapter provides a general summary of the scientific challenges addressed by this

thesis, as well as the conclusions drawn from each Chapter. Possibilities for future work which builds upon the contributions of this thesis are identified. 


\subsection{Scientific Challenges Addressed in this Thesis}

This thesis explored numerous challenges related to using Monte Carlo model based dose calculations for low-energy permanent implant brachytherapy by studying the impact of necessary virtual patient modelling assumptions. Retrospective dose calculations using clinical permanent implant prostate and breast brachytherapy patient data were performed to gain a better understanding of how real dose distributions may differ from those estimated using the water-based AAPM TG-43 clinical dose calculation paradigm. The Monte Carlo dose calculations were applied to radiobiological models to explore how differences in dose calculations translate to estimations of treatment efficacy, and to identify limitations of current radiobiological models which should be addressed before model based dose calculations are routinely adopted.

Accurate Monte Carlo dose calculations require accurate virtual patient geometry models. At the time of this thesis, the most pragmatic approach to derive these patient models is from static post-implant CT images which are normally acquired in the clinic for typical TG-43 evaluation. However, these CT images are burdened by metallic seed artifacts and provide limited soft tissue contrast which challenges tissue assignment. The low-energy brachytherapy sources studied in this thesis result in primarily photoelectric photon interactions causing a substantial cross section dependence on simulated tissue effective atomic number. A better understanding of the sensitivity of the Monte Carlo dose calculations to patient modelling assumptions is necessary before model based dose calculations can be confidently applied clinically or results can be fairly compared between scientific studies. 


\subsection{Summary of Chapter Conclusions}

\subsubsection{Chapter 3 - Metallic Artifact Reduction}

Chapter 3 used a general homogeneous brachytherapy phantom and eight example permanent implant prostate brachytherapy cases to explore four general approaches to mitigate metallic artifacts from post brachytherapy treatment $\mathrm{CT}$ images. The artifact mitigation methods were: a thresholding approach, a spatial median filter and two sinogram approaches, one using a virtual simulated sinogram and one using the raw CT scanner sinogram.

It is possible to avoid the issue of artifact mitigation altogether by developing virtual patient models with uniform nominal tissue mass densities, but this Chapter showed that nominal density models can lead to target volume dose metric inaccuracies of several percent (Table 3.7). Thus, the most accurate patient models

will require heterogeneous tissue densities based on CT number, necessitating the removal of metallic artifacts from post-implant CT images.

Three of the four artifact mitigation methods (STR, median filter and virtual sinogram) only require the reconstructed CT image which makes them more useful than the raw sinogram method for retrospective analysis since the raw scanner data are not often archived. No method eliminated all artifacts in all cases, and the remaining or newly introduced imaging artifacts were different for each approach (Figure 3.2). We found no apparent advantage to the raw sinogram method over the simpler image-based methods. By restricting the STR and median filter to only affect image voxels surrounding the seeds, real tissue heterogeneities such as prostatic calcifications were better preserved (Figure 3.4). There is an interdependence of artifact mitigation method and tissue assignment scheme: more complex tissue assignment 
models, e.g., with small variations in CT number mapping to different tissues, are more sensitive to the differences between the applied MAR method (Table 3.7). We conclude that the seed guided methods, STR and median filter, may be most practical to apply in future work. We caution that the STR is limited by its inability to mitigate low CT number artifacts and the median filter may smooth CT numbers preventing the differentiation of soft tissues represented by similar CT number (Figure 3.2).

\subsubsection{Chapter 4 - Breast Dose Calculations}

Chapter 4 presented retrospective dose calculations of four permanent breast seed implant patients for the purpose of exploring differences between water-based and full tissue model dose calculations and estimating the sensitivity of Monte Carlo dose calculations to various patient modelling assumptions.

We found that dose calculations were fairly insensitive to metallic artifact reduction method (Figure 4.5), except when artifact mitigation changed the relative assignment of adipose and gland tissues in the breast model (characteristic of median filter). With respect to tissue assignment, we observed that separately assigning adipose and gland compared to estimating soft tissue composition as a mixture of the two tissues yielded up to $20 \%$ differences in local voxel doses and differences to target dose metrics of several percent (Figure 4.6 and Table 4.4). Employing alternate published tissue compositions for target volume and skin tissues can change target and skin dose metrics by over 25\% (Figure 4.7 and Table 4.4), suggesting a need to adopt consensus compositions such as those listed in TG-186. The substantial sensitivity of full tissue model Monte Carlo dose calculations to patient modelling approach motivates the adoption of a consensus modelling approach to ensure consistency of 
practice and allow for better comparisons between studies.

This Chapter identified that TG-43 local doses and DVH metrics are overestimated in the target volume and underestimated in the skin by tens of percent compared to more detailed full-tissue Monte Carlo models (Figure 4.8). However, the differences between simulated TG-43 and detailed Monte Carlo model dose calculations varied considerably between patients due to their substantial differences in treatment site geometry (Table 4.4). Future work is needed to explore breast brachytherapy dose calculations in a larger cohort of patients before a reassessment of prescription dose and skin dose thresholds can be justified.

\subsubsection{Chapter 5 - Prostate Dose Calculations}

Chapter 5 included a large scale retrospective dosimetry analysis of 613 permanent implant prostate brachytherapy patients and a sensitivity analysis in a cohort of calcified and non-calcified patients with respect to tissue composition and approach to model calcifications.

TG-43 and full tissue model Monte Carlo dose calculations varied considerably across the patient cohort (Figure 5.2), but average target and organ-at-risk dose metrics were several percent different between dose calculation models, with higher dose metrics generally estimated using the TG-43 water-based model (Table 5.3). The largest differences in dose calculations were observed in patients with prostatic calcifications, leading to differences in target $D_{90}$ of up to $25 \%$ between TG-43 and full tissue dose calculations (Figure 5.4). Since current clinical practice estimates treatment quality based on post treatment dosimetry, this work motivates the adoption of more accurate model based dose calculations for clinical use, especially for patients with prostatic calcifications. 
We observed a substantial sensitivity of Monte Carlo prostate brachytherapy dose calculations to virtual patient modelling assumptions (Figure 5.5). The choice of organ-at-risk tissue composition has a notable effect only to doses scored in that tissue (ie. rectum tissue composition does not affect bladder doses). However, the target volume tissue modelling approach can affect all organ-at-risk doses since most simulated particles must first traverse the target volume before reaching the organsat-risk. The method to model calcifications (ie. as a solid tissue, or as homogeneous mixtures of prostate and calcification to simulate micro or diffuse calcifications) and the composition of the calcifications, only affect the approximate $10 \%$ of patients who have prostatic calcifications. Most prostate brachytherapy studies model calcifications using a composition from the breast, but we observed that this assumption resulted in "middle-of-the-pack" dose calculations compared to models assuming more realistic prostatic calcification compositions, suggesting that this widely-adopted approximation is reasonable. To assure consistency of practice, a consensus permanent implant prostate brachytherapy patient modelling approach such as the MCref described in Chapter 5, should be adopted in future studies.

\subsubsection{Chapter 6 - Prostate Radiobiological Modelling}

Chapter 6 applied the large cohort of prostate brachytherapy dose calculations from Chapter 5 to existing radiobiological models to explore how differences in waterbased and full tissue Monte Carlo dose calculations may affect estimates of biological outcomes.

There are a variety of published and adopted radiobiological models for permanent implant prostate brachytherapy, and by systematically comparing them using the same dose calculations and assumed biological parameters, we identify a 
substantial variation in estimates of biological dose and tumour control probabilities between models (Figure 6.3). Differences between water-based TG-43 and full tissue Monte Carlo dose calculations yield differences in biological doses of a similar order as differences in physical dose, and differences in tumour control probabilities which can be substantial for low biological doses. While most of the calculated tumour control probabilities are below expected values based on outcomes studies, these may be adjusted by using steeper more realistic tumour control probability functions (Table 6.3) and by adopting different physical parameters (Figure 6.6). More advanced biological dose models must account for the highly heterogeneous doses characteristic of permanent implant brachytherapy, but current models rely on rejecting low-dose volumes which is problematic when coupled with full tissue Monte Carlo dose calculations that can identify calcification shielding effects (Figure 6.1). We proposed three general approaches to extend current biological dose models to help differentiate those low doses of interest caused by calcification shielding, but we found that these altered models caused substantial differences only in select highly calcified patients (Table 6.3). Nevertheless, these extended heterogeneous dose radiobiological models were better at accounting for differences in water-based and full tissue Monte Carlo dose calculations compared to existing models (Figure 6.4).

\subsubsection{Outlook and Future Research Possibilities}

Many of the virtual patient modelling challenges addressed in this thesis are motivated by the need to derive patient models from the available post-implant CT images which are collected under standard TG-43 work flow. In the future, virtual patient models may be derived from post-implant images acquired using more advanced imaging modalities such as dual energy CT or MRI, which may allow for better soft tissue differentiation. However, few Canadian centres currently image prostate brachyther- 
apy patients with these technologies and no study has demonstrated that Monte Carlo dose calculations using the resulting virtual patient models are different than those derived from standard post-implant CTs. A fruitful future research endeavour would be to correlate dose calculations from CT-based models with those using virtual patient models derived from advanced imaging modalities. Additionally, the Monte Carlo dose calculations presented in this thesis are based on single post-implant images and future work is needed to develop robust 4D brachytherapy Monte Carlo models which account for swelling and edema and explore the differences in dose calculations between these static and dynamic conditions.

This thesis has shown that low energy brachytherapy Monte Carlo dose calculations have a sensitivity to assumed tissue assignment mapping and tissue compositions of the same order as differences between water-based and full tissue Monte Carlo dose calculations. This strongly motivates the definition and wide-spread adoption of consensus reference modelling schemes to allow for better comparisons between studies.

This thesis has presented the largest cohort of retrospective permanent implant prostate brachytherapy Monte Carlo dose calculations to-date. In the future, these dosimetry data will be correlated to physician defined treatment outcomes based on continued patient followup. Potentially, stronger correlations may be found between outcomes and model based dose calculations, compared to water-based dose calculations, which would further motivate the adoption of Monte Carlo for clinical use and justify a reassessment of prescription and organ-at-risk threshold doses.

Coupling Monte Carlo permanent implant brachytherapy dose calculations with existing radiobiological models will necessitate that the effects of low-dose volumes due to tissue heterogeneities are considered when estimating biological effect. Moreover, meaningful radiobiological modelling outcomes for specific patients will re- 
quire the knowledge of where tumour cell clusters are located throughout the target volume. As clinics continue to adopt more advanced diagnostic imaging modalities, such as multi-parametric MRI, this data may become available to researchers and will enable substantive advancements in radiobiological modelling. 


\section{References}

${ }^{1}$ Canadian Cancer Society Advisory Committee on Cancer Statistics. Canadian cancer statistics 2015. Canadian Cancer Society, 2015.

${ }^{2}$ J. J. Mazeron, P. Scalliet, E. Van Limbergen, and E. Lartigau. Radiobiology of brachytherapy and the dose-rate effect. The GEC-ESTRO handbook of brachytherapy. Brussels: ESTRO, pages 95-121, 2002.

${ }^{3}$ E. B. Podgorsak. Review of radiation oncology physics: a handbook for teachers and students. Vienna, International Atomic Energy Agency. Educational reports series, 2003.

${ }^{4}$ R. E. P. Taylor and D. W. O. Rogers. An EGSnrc Monte Carlo-calculated database of TG-43 parameters. Med. Phys., 35:4228 - 4241, 2008.

${ }^{5}$ M. Keyes, J. Crook, W. J. Morris, G. Morton, T. Pickles, N. Usmani, and E. Vigneault. Canadian prostate brachytherapy in 2012. Can Urol Assoc J, 7:51-58, February 2013.

${ }^{6}$ P. Grimm, I. Billiet, D. Bostwick, A. P. Dicker, S. Frank, J. Immerzeel, M. Keyes, P. Kupelian, W. R. Lee, S. Machtens, J. Mayadev, B. J. Moran, G. Merrick, J. Millar, M. Roach, R. Stock, K. Shinohara, M. Scholz, E. Weber, A. Zietman, M. Zelefsky, J. Wong, S. Wentworth, R. Vera, and S. Langley. Comparative analysis of 
prostate-specific antigen free survival outcomes for patients with low, intermediate and high risk prostate cancer treatment by radical therapy. results from the prostate cancer results study group. BJU International, 109:22-29, 2012.

${ }^{7}$ K. A. Hinnen, J. J. Battermann, J. G. H. van Roermund, M. A. Moerland, I. M. Jürgenliemk-Schulz, S. J. Frank, and M. van Vulpen. Long-term biochemical and survival outcome of 921 patients treated with i-125 permanent prostate brachytherapy. International Journal of Radiation Oncology* Biology* Physics, 76(5):1433$1438,2010$.

${ }^{8}$ M. A. Moyad, G. S. Merrick, W. M. Butler, K. E. Wallner, R. W. Galbreath, B. Kurko, and E. Adamovich. Statins, especially atorvastatin, may favorably influence clinical presentation and biochemical progression-free survival after brachytherapy for clinically localized prostate cancer. Urology, 66(6):1150-1154, 2005.

${ }^{9}$ E. Martinez, A. Daidone, C. Gutierrez, J. Pera, A. Boladeras, F. Ferrer, F. Pino, J. F. Suarez, A. Polo, and F. Guedea. Permanent seed brachytherapy for clinically localized prostate cancer: Long-term outcomes in a 700 patient cohort. Brachytherapy, 14(2):166-172, 2015.

${ }^{10}$ G. S. Merrick, W. M. Butler, K. E. Wallner, R. W. Galbreath, Z. A. Allen, and E. Adamovich. Androgen-deprivation therapy does not impact cause-specific or overall survival after permanent prostate brachytherapy. International Journal of Radiation Oncology* Biology* Physics, 65(3):669-677, 2006.

${ }^{11}$ D. C. Farrow, W. C. Hunt, and J. M. Samet. Geographic variation in the treatment of localized breast cancer. New England Journal of Medicine, 326(17):1097-1101, 1992. 
${ }^{12}$ J. Fisher, C. Scott, R. Stevens, B. Marconi, L. Champion, G.M. Freedman, F. Asrari, M.V. Pilepich, J.D. Gagnon, and G. Wong. Randomized phase III study comparing best supportive care to biafine as a prophylactic agent for radiationinduced skin toxicity for women undergoing breast irradiation: Radiation therapy oncology group RTOG 97-13. International Journal of Radiation Oncology Biology Physics, 48(5):1307-1310, 2000.

${ }^{13}$ J. P. Pignol, B. Keller, E. Rakovitch, R. Sankreacha, H. Easton, and W. Que. First report of a permanent breast ${ }^{103} \mathrm{Pd}$ seed implant as adjuvant radiation treatment for early-stage breast cancer. Int. J. Radiat. Oncol. Biol. Phys., 64:176 - 181, 2006.

${ }^{14}$ F.A. Vicini, L. Kestin, P. Chen, P. Benitez, and N.S. Goldstein. Limited-field radiation therapy in the management of early-stage breast cancer. Journal of the National Cancer Institute, 95(16):1205-1211, 2003.

${ }^{15}$ J. P. Pignol, J. M. Caudrelier, J. Crook, C. McCann, P. Truong, and H. A. Verkooijen. Report on the clinical outcomes of permanent breast seed implant for early-stage breast cancers. International Journal of Radiation Oncology*Biology*Physics, 93(3):614-621, 2015.

${ }^{16}$ M. J. Zelefsky and J. Whitmore. Long-term results of retropubic permanent 125 iodine implantation of the prostate for clinically localized prostatic cancer. The Journal of urology, 158(1):23-30, 1997.

${ }^{17}$ R. Nath, K. Roberts, M. Ng, R. Peschel, and Z. Chen. Correlation of medical dosimetry quality indicators to the local tumor control in patients with prostate cancer treated with iodine-125 interstitial implants. Medical physics, 25(12):2293$2307,1998$. 
${ }^{18}$ R. G. Stock, N. N. Stone, A. Tabert, C. Iannuzzi, and J. K. DeWyngaert. A dose response study for I-125 prostate implants. International Journal of Radiation Oncology*Biology*Physics, 41(1):101 - 108, 1998.

${ }^{19}$ R. Nath, L. L. Anderson, G. Luxton, K. A. Weaver, J. F. Williamson, and A. S. Meigooni. Dosimetry of interstitial brachytherapy sources: Recommendations of the AAPM Radiation Therapy Committee Task Group No. 43. Med. Phys., 22:209 - 234, 1995.

${ }^{20}$ M. J. Rivard, B. M. Coursey, L. A. DeWerd, M. S. Huq, G. S. Ibbott, M. G. Mitch, R. Nath, and J. F. Williamson. Update of AAPM Task Group No. 43 Report: A revised AAPM protocol for brachytherapy dose calculations. Med. Phys., 31:633 $-674,2004$.

${ }^{21}$ M. J. Rivard, W. M. Butler, L. A. DeWerd, M. S. Huq, G. S. Ibbott, A. S. Meigooni, C. S. Melhus, M. G. Mitch, R. Nath, and J. F. Williamson. Supplement to the 2004 update of the AAPM Task Group No. 43 Report. Med. Phys., 34:2187 $-2205,2007$.

${ }^{22}$ M. J. Rivard, D. A. R. Evans, and I. Kay. A technical evaluation of the nucletron FIRST system: Conformance of a remote afterloading brachytherapy seed implantation system to manufacturer specifications and AAPM task group report recommendations. Journal of Applied Clinical Medical Physics, 6(1), 2005.

${ }^{23}$ J. F. Corbett, J. J. Jezioranski, J. Crook, T. Tran, and I. W. T. Yeung. The effect of seed orientation deviations on the quality of 125 I prostate implants. Physics in Medicine and Biology, 46(11):2785, 2001.

${ }^{24}$ C. A. Collins Fekete, M. Plamondon, A. G. Martin, E. Vigneault, F. Verhaegen, and L. Beaulieu. Quantifying the effect of seed orientation in postplanning dosimetry of low-dose-rate prostate brachytherapy. Med. Phys., 41(10), 2014. 
${ }^{25}$ M. J. Rivard, J. L. M. Venselaar, and L. Beaulieu. The evolution of brachytherapy treatment planning. Med. Phys., 36:2136 - 2153, 2009.

${ }^{26}$ L. Beaulieu, A. C. Tedgren, J. F. Carrier, S. D. Davis, F. Mourtada, M. J. Rivard, R. M. Thomson, F. Verhaegen, T. A. Wareing, and J. F. Williamson. Report of the Task Group 186 on model-based dose calculation methods in brachytherapy beyond the TG-43 formalism: Current status and recommendations for clinical implementation. Med. Phys., 39:6208 - 6236, 2012.

${ }^{27}$ F. Ballester, A. C. Tedgren, D. Granero, A. Haworth, F. Mourtada, G. P. Fonseca, K. Zourari, P. Papagiannis, M. J. Rivard, and F. Siebert. A generic high-dose rate 192ir brachytherapy source for evaluation of model-based dose calculations beyond the TG-43 formalism. Medical physics, 42(6):3048-3062, 2015.

${ }^{28}$ M. Abdoli, M. R. Ay, A. Ahmadian, R. A. J. O. Dierckx, and H. Zaidi. Reduction of dental filling metallic artifacts in CT-based attenuation correction of PET data using weighted virtual sinograms optimized by a genetic algorithm. Med. Phys., $37(12): 6166-6177,2010$.

${ }^{29}$ J. G. H. Sutherland, K. M. Furutani, Y. I. Garces, and R. M. Thomson. Modelbased dose calculations for ${ }^{125}$ I lung brachytherapy. Med. Phys., 39:4365 -4377, 2012.

${ }^{30}$ J. G. H. Sutherland, N. Miksys, K. M. Furutani, and R. M. Thomson. Metallic artifact mitigation and organ-constrained tissue assignment for monte carlo calculations of permanent implant lung brachytherapy. Med. Phys., 41(1):011712, 2014.

${ }^{31}$ C. Xu, F. Verhaegen, D. Laurendeau, S. A. Enger, and L. Beaulieu. An algorithm for efficient metal artifact reductions in permanent seed implants. Med. Phys., $38: 47-56,2011$. 
${ }^{32}$ O. Watzke and W. A. Kalender. A pragmatic approach to metal artifact reduction in CT: merging of metal artifact reduced images. European Radiology, 14(5):849856, 2004.

${ }^{33}$ M. Yazdi, M. A. Lari, G. Bernier, and L. Beaulieu. An opposite view data replacement approach for reducing artifacts due to metallic dental objects. Med. Phys., 38(4):2275-2281, 2011.

${ }^{34}$ M. Yazdi, L. Gingras, and L. Beaulieu. An adaptive approach to metal artifact reduction in helical computed tomography for radiation therapy treatment planning: Experimental and clinical studies. Int. J. Radiat. Oncol. Biol. Phys., 62:1224$1231,2005$.

${ }^{35}$ E. Meyer, R. Raupach, M. Lell, B. Schmidt, and M. Kachelrie. Normalized metal artifact reduction (NMAR) in computed tomography. Med. Phys., 37(10):5482$5493,2010$.

${ }^{36}$ M. Bazalova, L. Beaulieu, S. Palefsky, and V. Verghaegen. Correction of CT artifacts and its influence on Monte Carlo dose calculations. Med. Phys., 34(6):2119 $-2132,2007$.

${ }^{37}$ M. Abdoli, J. R. Jong, J. Pruim, R. J. O. Dierckx, and H. Zaidi. Reduction of artefacts caused by hip implants in CT-based attenuation-corrected pet images using 2-D interpolation of a virtual sinogram on an irregular grid. European Journal of Nuclear Medicine and Molecular Imaging, 38(12):2257-2268, 2011.

${ }^{38}$ P. S. Basran, A. Robertson, and D. Wells. CT image artifacts from brachytherapy seed implants: A postprocessing 3D adaptive median filter. Med. Phys., 38:712 $718,2011$.

${ }^{39}$ Y. Takahashi, S. Mori, T. Kozuka, K. Gomi, T. Nose, T. Tahara, M. Oguchi, and T. Yamashita. Preliminary study of correction of metal artifacts due to I-125 seeds 
in postimplant dosimetry for prostate permanent implant brachytherapy. Radiat. Med., 24:133 - 138, 2006.

${ }^{40}$ W. Schneider, T. Bortfeld, and W. Schlegel. Correlation between CT numbers and tissue parameters needed for Monte Carlo simulations of clinical dose distributions. Phys. Med. Biol., 45:459-478, 2000.

${ }^{41}$ J. Crook, N. Patil, C. Ma, M. McLean, and J. Borg. Magnetic resonance imagingdefined treatment margins in iodine-125 prostate brachytherapy. International Journal of Radiation Oncology*Biology*Physics, 77(4):1079 - 1084, 2010.

${ }^{42}$ H. Q. Woodard and D. R. White. The composition of body tissues. Brit. J. Radiol., 59:1209 - 1219, 1986.

${ }^{43}$ D. R. White, H. Q. Woodard, and S. M. Hammond. Average soft-tissue and bone models for use in radiation dosimetry. Brit. J. Radiol., 60:907 - 913, 1987.

${ }^{44}$ ICRP. Report of Task Group on Reference Man. ICRP Report 23, ICRP, Washington D.C., 1975.

${ }^{45}$ ICRU. Photon, Electron, Photon and Neutron Interaction Data for Body Tissues. ICRU Report 46, ICRU, Washington D.C., 1992.

${ }^{46}$ ICRP. Basic Anatomical and Physiological Data for Use in Radiological Protection: Reference Values. Annals of the ICRP 89, ICRP, Washington D.C., 2003.

${ }^{47}$ J. G. H. Sutherland, R. M. Thomson, and D. W. O. Rogers. Changes in dose with segmentation of breast tissues in Monte Carlo calculations for low-energy brachytherapy. Med. Phys., 38:4858 - 4865, 2011.

${ }^{48}$ H. Afsharpour, G. Landry, B. Reniers, J. P. Pignol, L. Beaulieu, and F. Verhaegen. Tissue modeling schemes in low energy breast brachytherapy. Phys. Med. Biol., 56:7045 - 7060, 2011. 
${ }^{49}$ F. H. Attix. Introduction to Radiological Physics and Radiation Dosimetry. Wiley, New York, 1986.

${ }^{50}$ A. Ahnesjö. Collapsed cone convolution of radiant energy for photon dose calculation in heterogeneous media. Med. Phys., 16:577 - 592, 1989.

${ }^{51}$ A. C. Tedgren and A. Ahnesjö. Optimization of the computational efficiency of a 3D, collapsed cone dose calculation algorithm for brachytherapy. Medical physics, 35(4):1611-1618, 2008.

${ }^{52}$ K. A. Gifford, M. J. Price, J. L. Horton Jr, T. A. Wareing, and F. Mourtada. Optimization of deterministic transport parameters for the calculation of the dose distribution around a high dose-rate ir192 brachytherapy source. Medical physics, $35(6): 2279-2285,2008$.

${ }^{53}$ K. Zourari, E. Pantelis, A. Moutsatsos, L. Sakelliou, E. Georgiou, P. Karaiskos, and P. Papagiannis. Dosimetric accuracy of a deterministic radiation transport based 192ir brachytherapy treatment planning system. part III. comparison to monte carlo simulation in voxelized anatomical computational models. Medical physics, 40(1):011712, 2013.

${ }^{54}$ O. Chibani and J. F. Williamson. Mcpi: A sub-minute monte carlo dose calculation engine for prostate implants. Med. Phys., 32:3688 - 3698, 2005.

${ }^{55}$ H Afsharpour, G Landry, M D’Amours, S Enger, B Reniers, E Poon, J-F Carrier, F Verhaegen, and L Beaulieu. ALGEBRA: ALgorithm for the heterogeneous dosimetry based on GEANT4 for BRAchytherapy. Phys. Med. Biol., 57(11):3273 $-\mathrm{i} 3280,2012$.

${ }^{56}$ S. Agostinelli et al. GEANT4 - a simulation toolkit. Nucl. Inst. Meth. A, 506:250 $-303,2003$. 
${ }^{57}$ R. E. P. Taylor, G. Yegin, and D. W. O. Rogers. Benchmarking BrachyDose: voxel-based EGSnrc Monte Carlo calculations of TG-43 dosimetry parameters. Med. Phys., 34:445 - 457, 2007.

${ }^{58}$ R M Thomson, G Yegin, R E P Taylor, J G H Sutherland, and D W O Rogers. Fast Monte Carlo dose calculations for brachytherapy with BrachyDose. Med. Phys., 37:(abstract) $3910-3911,2010$.

${ }^{59}$ I. Kawrakow and D. W. O. Rogers. The EGSnrc Code System: Monte Carlo simulation of electron and photon transport. Technical Report PIRS701, National Research Council of Canada, Ottawa, Canada, page see http://www.irs.inms.nrc.ca/inms/irs/EGSnrc/EGSnrc.html, 2000.

${ }^{60}$ I. Kawrakow. Accurate condensed history Monte Carlo simulation of electron transport. I. EGSnrc, the new EGS4 version. Med. Phys., 27:485 - 498, 2000.

${ }^{61} \mathrm{G}$. Yegin. A new approach to geometry modelling of Monte Carlo particle transport: an application to EGS. Nucl. Inst. Meth. B, 211:331 - 338, 2003.

${ }^{62}$ M. J. Berger. ESTAR, PSTAR and ASTAR: Computer Programs for Calculating Stopping-Power and Ranges for Electrons, Protons, and Helium Ions. NIST Report NISTIR-4999 (Washington DC), available on-line at http://physics.nist.gov/Star, 1992.

${ }^{63}$ F. Ballester, D. Granero, J. Pérez-Calatayud, C. S. Melhus, and M. J. Rivard. Evaluation of high-energy brachytherapy source electronic disequilibrium and dose from emitted electrons. Med. Phys., 36:4250 - 4256, 2009.

${ }^{64}$ C. C. Ling and E. D. Yorke. Interface dosimetry for i-125 sources. Medical physics, 16(3):376-381, 1989. 
${ }^{65}$ J. F. Williamson. Monte Carlo evaluation of kerma at a point for photon transport problems. Med. Phys., 14:567 - 576, 1987.

${ }^{66}$ R. Nath, W. S. Bice, W. M. Butler, Z. Chen, A. S. Meigooni, V. Narayana, M. J. Rivard, and Y. Yu. AAPM recommendations on dose prescription and reporting methods for permanent interstitial brachytherapy for prostate cancer: Report of Task Group 137. Med. Phys., 36:5310 - 5322, 2009.

${ }^{67}$ R. G. Dale. The application of the linear-quadratic dose-effect equation to fractionated and protracted radiotherapy. Int. J. Radiat. Oncol. Biol. Phys., 58(690):515 $-528,1985$.

${ }^{68} \mathrm{R}$ G Dale. Radiobiological assessment of permanent implants using tumour repopulation factors in the linear-quadratic model. Brit. J. Radiol., 62:241 - 244, 1989.

${ }^{69} \mathrm{~S}$. Webb and A. E. Nahum. A model for calculating tumour control probability in radiotherapy including the effects of inhomogeneous distributions of dose and clonogenic cell density. Physics in Medicine and Biology, 38(6):653, 1993.

${ }^{70}$ I. Kawrakow and D. W. O. Rogers. The EGSnrc Code System: Monte Carlo simulation of electron and photon transport. NRC Technical Report PIRS-701 v4-2-2-5, National Research Council of Canada, Ottawa, Canada. http://www.irs.inms.nrc.ca/inms/irs/EGSnrc/EGSnrc.html, 2007.

${ }^{71}$ M. J. Berger and J. H. Hubbell. XCOM: Photon cross sections on a personal computer. Report NBSIR87-3597, National Institute of Standards Technology (NIST), Gaithersburg, MD 20899, U.S.A., 1987.

72 J. H. Hubbell. Photon Mass Attenuation and Energy-absorption Coefficients from $1 \mathrm{keV}$ to $20 \mathrm{MeV}$. Int. J. Appl. Radiat. Isot., 33:1269-1290, 1982. 
${ }^{73}$ H. E. Johns. The physics of radiology 4th ed. Springfield, IL: Charles, 1983.

${ }^{74}$ B. R. B. Walters and D. W. O. Rogers. DOSXYZnrc Users Manual. NRC Report PIRS 794, 2002.

${ }^{75}$ B. R. B. Walters, I. Kawrakow, and D. W. O. Rogers. History by history statistical estimators in the BEAM code system. Med. Phys., 29:2745 - 2752, 2002.

${ }^{76}$ D. W. O. Rogers, I. Kawrakow, J. P. Seuntjens, and B. R. B. Walters. NRC User Codes for EGSnrc. NRC Technical Report PIRS-702, National Research Council of Canada, Ottawa, Canada. http://www.irs.inms.nrc.ca/inms/irs/EGSnrc/EGSnrc.html, 2000.

${ }^{77}$ I. J. Chetty et al. Report of the AAPM Task Group No. 105: Issues associated with clinical implementation of Monte Carlo-based photon and electron external beam treatment planning. Med. Phys., 34:4818 -4853, 2007.

78 J.-F. Carrier, M. D’Amours, F. Verhaegen, B. Reniers, A.-G. Martin, E. Vigneault, and L. Beaulieu. Postimplant dosimetry using a Monte Carlo dose calculation engine: a new clinical standard. Int. J. Radiat. Oncol. Biol. Phys., 68:1190-1198, 2007.

${ }^{79}$ B. J. Davis, E. M. Horwitz, W. R. Lee, J. M. Crook, R. G. Stock, G. S. Merrick, W. M. Butler, P. D. Grimm, N. N. Stone, L. Potters, A. L. Zietman, and M. J. Zelefsky. American brachytherapy society consensus guidelines for transrectal ultrasound-guided permanent prostate brachytherapy. Brachytherapy, 11(1):6 - 19, 2012. Special Issue: American Brachytherapy Society Guidelines for Prostate and Gynecology.

${ }^{80}$ H. Afsharpour, J. P. Pignol, B. K. Keller, J. F. Carrier, B. Reniers, F. Verhaegen, and L. Beaulieu. Influence of breast composition and interseed attenuation in dose 
calculations for post-implant assessment of permanent breast $103 \mathrm{Pd}$ seed implant. Phys. Med. Biol., 55:4547-4561, 2010.

${ }^{81}$ G. Landry, B. Reniers, L. Murrer, L. Lutgens, E. Bloemen-Van Gurp, J. P. Pignol, B. Keller, L. Beaulieu, and F Verhaegen. Sensitivity of low energy brachytherapy Monte Carlo dose calculations to uncertainties in human tissue composition. Med. Phys., 37:5188 - 5198, 2010.

${ }^{82}$ C. A. Collins Fekete, M. Plamondon, A. G. Martin, E. Vigneault, F. Verhaegen, and L. Beaulieu. Calcifications in low-dose rate prostate seed brachytherapy treatment: Post-planning dosimetry and predictive factors. Radiotherapy and Oncology, (0), 2015.

${ }^{83}$ D. Pope. The dosimetric effect of prostatic calcification on Low Dose Rate (LDR) brachytherapy. MSc Thesis, University of Wollongong, 2013.

${ }^{84}$ N. Miksys, C. Xu, L. Beaulieu, and R. M. Thomson. Development of virtual patient models for permanent implant brachytherapy Monte Carlo dose calculations: interdependence of CT image artifact mitigation and tissue assignment. Physics in Medicine and Biology, 60:6039-6062, 2015.

${ }^{85}$ C. Furstoss, B. Reniers, M. J. Bertrand, E. Poon, J.-F. Carrier, B. M. Keller, J. P. Pignol, L. Beaulieu, and F. Verhaegen. Monte Carlo study of LDR seed dosimetry with an application in a clinical brachytherapy breast implant. Med. Phys., 36(5):1848-1858, 2009.

${ }^{86}$ M.J. Yaffe, J.M. Boone, N. Packard, O. Alonzo-Proulx, S.Y. Huang, C.L. Peressotti, A. Al-Mayah, and K. Brock. The myth of the 50-50 breast. Med. Phys., 36:5437 - 5443, 2009. 
${ }^{87}$ S. A. White, G. Landry, G. P. Fonseca, R. Holt, T. Rusch, L. Beaulieu, F. Verhaegen, and B. Reniers. Comparison of TG-43 and TG-186 in breast irradiation using a low energy electronic brachytherapy source. Med. Phys., 41(6), 2014.

${ }^{88}$ G. R. Hammerstein, D. W. Miller, D. R. White, M. E. Masterson, H. Q. Woodard, and J. S. Laughlin. Absorbed Radiation Dose in Mammography. Radiology, 130:485 - 491, 1979.

${ }^{89}$ M. Abdoli, M. R. Ay, A. Ahmadian, and H. Zaidi. A virtual sinogram method to reduce dental metallic implant artefacts in computed tomography-based attenuation correction for PET. Nuclear Medicine Communications, 31(1):22 - 31, 2010.

${ }^{90}$ H Afsharpour, B Reniers, G Landry, J.-P. Pignol, B M Keller, F Verhaegen, and L Beaulieu. Consequences of dose heterogeneity on the biological efficiency of ${ }^{103} \mathrm{Pd}$ permanent breast seed implants. Phys. Med. Biol., 57(3):809-823, 2012.

${ }^{91}$ G. Landry, B. Reniers, J. P. Pignol, L. Beaulieu, and F. Verhaegen. The difference of scoring dose to water or tissues in Monte Carlo dose calculations for low energy brachytherapy photon sources. Med. Phys., 38:1526 - 1533, 2011.

${ }^{92}$ I. Kawrakow and D. W. O. Rogers. The EGSnrc Code System: Monte Carlo simulation of electron and photon transport. Technical Report PIRS-701, National Research Council of Canada, Ottawa, Canada, 2000.

${ }^{93}$ ICRU. Dose and Volume Specification for Reporting Interstitial Therapy. ICRU Report 58, ICRP, Washington D.C., 1998.

94 T. Major, G. Frohlich, and C. Polgar. Assessment of dose homogeneity in conformal interstitial breast brachytherapy with special respect to ICRU recommendations. Journal of Contemporary Brachytherapy, 3(3):150-155, 2011. 
${ }^{95}$ O. Chibani, J. F. Williamson, and D. Todor. Dosimetric effects of seed anisotropy and interseed attenuation for ${ }^{103} \mathrm{Pd}$ and ${ }^{125} \mathrm{I}$ prostate implants. Med. Phys., 32:2557 - 2566, 2005.

96 J.-F. Carrier, L. Beaulieu, F. Therriault-Proulx, and R. Roy. Impact of interseed attenuation and tissue composition for permanent prostate implants. Med. Phys., $33: 595-604,2006$

${ }^{97}$ H. Afsharpour, M. D'Amours, B. Coté, J.-F. Carrier, F. Verhaegen, and L. Beaulieu. A Monte Carlo study on the effect of seed design on the interseed attenuation in permanent prostate implants. Med. Phys., 35:3671 - 3681, 2008.

${ }^{98}$ M. Ghorbani, F. Salahshour, A. Haghparast, T. A. Moghaddas, and C. Knaup. Effect of tissue composition on dose distribution in brachytherapy with various photon emitting sources. Journal of contemporary brachytherapy, 6(1):5467, March 2014 .

99 J. Mason, B. Al-Qaisieh, P Bownes, A Henry, and D. Thwaites. Investigation of interseed attenuation and tissue composition effects in 125i seed implant prostate brachytherapy. Brachytherapy, 13(6):603 - 610, 2014.

${ }^{100}$ A. Youssef and W. Tawadrous. Does intraprostatic calcifications affect low-doserate brachytherapy? Brachytherapy, $7(2): 184-185,2008$.

${ }^{101}$ J. H. Suh, J. M. Gardner, K. H. Kee, S. Shen, A. G. Ayala, and J. Y. Ro. Calcifications in prostate and ejaculatory system: a study on 298 consecutive whole mount sections of prostate from radical prostatectomy or cystoprostatectomy specimens. Annals of Diagnostic Pathology, 12(3):165 - 170, 2008.

102 C. G. Hong, B. I. Yoon, H. S. Choe, U. S. Ha, D. W. Sohn, and Y. H. Cho. The prevalence and characteristic differences in prostatic calcification between health 
promotion center and urology department outpatients. Korean J Urol, 53(5):330334, May 2012.

${ }^{103}$ D. J. Pope, D. L. Cutajar, S. P. George, S. Guatelli, J. A. Bucci, K. E. Enari, S. Miller, R. Siegele, and A. B. Rosenfeld. The investigation of prostatic calcifications using -pixe analysis and their dosimetric effect in low dose rate brachytherapy treatments using GEANT4. Physics in Medicine and Biology, 60(11):4335, 2015.

${ }^{104}$ S. M. Bentzen, L. S. Constine, J. O. Deasy, A. Eisbruch, A. Jackson, L. B. Marks, R. K. Ten Haken, and E. D. Yorke. Quantitative analyses of normal tissue effects in the clinic (QUANTEC): An introduction to the scientific issues. International Journal of Radiation Oncology*Biology*Physics, 76(3, Supplement):S3 - S9, 2010. Quantitative Analyses of Normal Tissue Effects in the Clinic.

${ }^{105}$ P. J. Hoskin, A. Colombo, A. Henry, P. Niehoff, T. P. Hellebust, F. A. Siebert, and G. Kovacs. GEC/ESTRO recommendations on high dose rate afterloading brachytherapy for localised prostate cancer: An update. Radiotherapy and Oncology, 107(3):325 - 332, 2013.

${ }^{106}$ F. M. Waterman, N. Yue, B. W. Corn, and A. P. Dicker. Edema associated with i-125 or pd-103 prostate brachytherapy and its impact on post-implant dosimetry: an analysis based on serial $\{\mathrm{CT}\}$ acquisition. International Journal of Radiation Oncology*Biology*Physics, 41(5):1069 - 1077, 1998.

${ }^{107}$ N. Yue, A. P. Dicker, R. Nath, and F. M. Waterman. The impact of edema on planning 125i and 103pd prostate implants. Medical Physics, 26(5):763-767, 1999.

${ }^{108}$ G. Leclerc, M. C. Lavalle, R. Roy, E. Vigneault, and L. Beaulieu. Prostatic edema in i125 permanent prostate implants: Dynamical dosimetry taking volume changes into account. Medical Physics, 33(3):574-583, 2006. 
109 N. Hünemohr, H. Paganetti, S. Greilich, O. Jäkel, and J. Seco. Tissue decomposition from dual energy ct data for me based dose calculation in particle therapy. Medical physics, 41(6):061714, 2014.

${ }^{110}$ M. Bazalova, J. F. Carrier, L. Beaulieu, and F. Verhaegen. Dual-energy CT-based material extraction for tissue segmentation in monte carlo dose calculations. Phys. Med. Biol., 53(9):2439, 2008.

${ }^{111}$ M. Bazalova, J. F. Carrier, L. Beaulieu, and F. Verhaegen. Tissue segmentation in monte carlo treatment planning: A simulation study using dual-energy CT images. Radiotherapy and Oncology, 86(1):93 - 98, 2008.

${ }^{112}$ G. Landry, P. V. Granton, B. Reniers, M. C. Ollers, L. Beaulieu, J. E. Wilderger, and F. Vergaegen. Simulation study on potential accuracy gains from dual energy CT tissue segmentation for low-energy brachytherapy Monte Carlo dose calculations. Phys. Med. Biol., 56(19):6257 - 6278, 2011.

${ }^{113}$ M. Zaider and G. N. Minerbo. Tumour control probability: a formulation applicable to any temporal protocol of dose delivery. Physics in Medicine and Biology, $45(2): 279,2000$.

${ }^{114}$ R. G. Stock, N. N. Stone, J. A. Cesaretti, and B. S. Rosenstein. Biologically effective dose values for prostate brachytherapy: Effects on PSA failure and posttreatment biopsy results. International Journal of Radiation Oncology*Biology*Physics, $64(2): 527-533,2006$.

${ }^{115}$ N. N. Stone, R. G. Stock, J. A. Cesaretti, and P. Unger. Local control following permanent prostate brachytherapy: Effect of high biologically effective dose on biopsy results and oncologic outcomes. International Journal of Radiation Oncology*Biology*Physics, 76(2):355 - 360, 2010. 
${ }^{116}$ E. F. Miles, J. W. Nelson, A. K. Alkaissi, S Das, R. W. Clough, G. Broadwater, M. S. Anscher, J. P. Chino, and J. R. Oleson. Biologically effective dose (BED) correlation with biochemical control after lowdose rate prostate brachytherapy for clinically low-risk prostate cancer. International Journal of Radiation Oncology*Biology*Physics, 77(1):139 - 146, 2010.

${ }^{117}$ W. M. Butler, R. R. Stewart, and G. S. Merrick. Evaluation of radiobiologic biochemical control in a large permanent prostate brachytherapy population from a single institution using AAPM TG-137 parameters. Brachytherapy, 10(1):16 $28,2011$.

${ }^{118}$ C. C. Ling, J. Roy, N. Sahoo, K. Wallner, and L. Anderson. Quantifying the effect of dose inhomogeneity in brachytherapy: Application to permanent prostatic implant with $125 \mathrm{i}$ seeds. International Journal of Radiation Oncology*Biology*Physics, 28(4):971 - 977, 1994.

${ }^{119}$ A. Haworth, M. Ebert, D. Waterhouse, D. Joseph, and G. Duchesne. Assessment of i-125 prostate implants by tumor bioeffect. International Journal of Radiation Oncology*Biology*Physics, 59(5):1405 - 1413, 2004.

${ }^{120}$ A. Haworth, M. Ebert, D. Waterhouse, D. Joseph, and G. Duchesne. Prostate implant evaluation using tumour control probability-the effect of input parameters. Phys. Med. Biol., 49:3649 - 3664, 2004.

${ }^{121}$ H. Afsharpour, S. Walsh, C.A. Collins Fekete, E. Vigneault, F. Verhaegen, and L. Beaulieu. On the sensitivity of / prediction to dose calculation methodology in prostate brachytherapy. International Journal of Radiation Oncology*Biology*Physics, 88(2):345 - 350, 2014.

${ }^{122}$ C. R. King, T. A. DiPetrillo, and D. E. Wazer. Optimal radiotherapy for prostate cancer: predictions for conventional external beam, imrt, and brachyther- 
apy from radiobiologic models. International Journal of Radiation Oncology*Biology*Physics, 46(1):165 - 172, 2000.

123 J. Z. Wang, M. Guerrero, and X. A. Li. How low is the alpha/beta ratio for prostate cancer? International Journal of Radiation Oncology*Biology*Physics, $55(1): 194-203,2003$.

${ }^{124} \mathrm{~S}$. Webb. Optimum parameters in a model for tumour control probability including interpatient heterogeneity. Physics in Medicine and Biology, 39(11):1895, 1994.

${ }^{125}$ M Zaider and L. Hanin. Biologically-equivalent dose and long-term survival time in radiation treatments. Physics in Medicine and Biology, 52(20):6355, 2007.

${ }^{126}$ A. Niemierko and M. Goitein. Implementation of a model for estimating tumor control probability for an inhomogeneously irradiated tumor. Radiotherapy and Oncology, 29(2):140 - 147, 1993.

${ }^{127}$ W. A. Tom and J. F. Fowler. On cold spots in tumor subvolumes. Medical Physics, 29(7):1590-1598, 2002.

${ }^{128}$ O Zebentout, R Apardian, L Beaulieu, F Harel, AG Martin, and E Vigneault. Clinical outcome of intermediate risk prostate cancer treated with iodine 125 monotherapy: The hotel-dieu of quebec experience. Cancer radiotherapie: journal de la Societe francaise de radiotherapie oncologique, 14(3):183-188, 2010.

${ }^{129}$ K. M. G. Haustermans, I. Hofland, H. Van Poppel, R. Oyen, W. Van de Voorde, A. C. Begg, and J. F. Fowler. Cell kinetic measurements in prostate cancer. International Journal of Radiation Oncology* Biology* Physics, 37(5):1067-1070, 1997.

${ }^{130}$ A. V. DAmico, A Davis, S. O. Vargas, A. A. Renshaw, M. Jiroutek, and J. P. Richie. Defining the implant treatment volume for patients with low risk prostate 
cancer: does the anterior base need to be treated? International Journal of Radiation Oncology*Biology*Physics, 43(3):587 - 590, 1999.

${ }^{131}$ J. E. McNeal, E. A. Redwine, F. S. Freiha, and T. A. Stamey. Zonal distribution of prostatic adenocarcinoma: correlation with histologic pattern and direction of spread. The American journal of surgical pathology, 12(12):897-906, 1988.

${ }^{132}$ H. Frimmel, L. Egevad, E. Bengtsson, and C. Busch. Modeling prostate cancer distributions. Urology, 54(6):1028 - 1034, 1999.

${ }^{133}$ S. Langley, H.U. Ahmed, B. Al-Qaisieh, D. Bostwick, L. Dickinson, F. G. Veiga, P. Grimm, S. Machtens, F. Guedea, and M. Emberton. Report of a consensus meeting on focal low dose rate brachytherapy for prostate cancer. BJU International, 109:7-16, 2012.

${ }^{134} \mathrm{~T}$ Wallace, I Avital, A Stojadinovic, BL Brücher, E Cote, and J Yu. Multiparametric mri-directed focal salvage permanent interstitial brachytherapy for locally recurrent adenocarcinoma of the prostate: a novel approach. J Cancer, 4(2):146-151, 2013. 